Florida International University FIU Digital Commons

$11-2-2015$

\title{
A Novel Hip Implant Using 3D Woven Composite Material - Design and Analysis
}

Hari Kishore Adluru

Florida International University, hadlu001@fiu.edu

DOI: $10.25148 /$ etd.FIDC000215

Follow this and additional works at: https://digitalcommons.fiu.edu/etd

Part of the Biomechanical Engineering Commons, and the Computer-Aided Engineering and Design Commons

\section{Recommended Citation}

Adluru, Hari Kishore, "A Novel Hip Implant Using 3D Woven Composite Material - Design and Analysis" (2015). FIU Electronic Theses and Dissertations. 2318.

https://digitalcommons.fiu.edu/etd/2318 


\section{FLORIDA INTERNATIONAL UNIVERSITY}

Miami, Florida

\section{A NOVEL HIP IMPLANT USING 3D WOVEN COMPOSITE MATERIAL - DESIGN}

\section{AND ANALYSIS}

A dissertation submitted in partial fulfillment of

the requirements for the degree of

DOCTOR OF PHILOSOPHY

in

MECHANICAL ENGINEERING

by

Hari Kishore Adluru 
To: Interim Dean Ranu Jung

College of Engineering and Computing

This dissertation, written by Hari Kishore Adluru, and entitled A Novel Hip Implant Using 3D Woven Composite Material - Design and Analysis, having been approved in respect to style and intellectual content, is referred to you for judgment.

We have read this dissertation and recommend that it be approved.

Arvind Agarwal

Kinzy Jones Jr.

Norman H. Munroe

W. Kinzy Jones, co-major Professor

Benjamin Boesl, co-major Professor

Date of Defense: November 2, 2015

The dissertation of Hari Kishore Adluru is approved.

Interim Dean Ranu Jung

College of Engineering and Computing

Dean Lakshmi N. Reddi

University Graduate School

Florida International University, 2015 


\section{DEDICATION}

Dedicated to my parents, wife, sister and brother -without their love, support and sacrifice this dissertation would not have been completed. 


\section{ACKNOWLEDGMENTS}

I thank my major advisor Dr. Benjamin Boesl, for his support throughout the span of my PhD program. Dr. Boesl helped me in developing the ability to think critically. He has always had my best interest and provided me with every opportunity to grow, both professionally and personally. I am grateful for all the kindness and patience that he has shown over the years.

I sincerely thank my co-major advisor Dr. Kinzy W. Jones for his continued support and critical guidance throughout my graduate program. He always provided me his time in-spite of his busy schedule to discuss any difficulties I faced, being it research or other academic concerns over the span of my PhD.

I also thank my other committee members Dr. Arvind Agarwal, Dr. Norman D.H. Munroe and Dr. Kinzy Jones Jr., for agreeing to be on my dissertation committee and guide me through the process as and when needed.

I thank Dr. Eric Zhou and his team at US Air Force Research Lab for providing VTMS and BSAM software to perform Microanalysis of Woven Composites. He has always been there patiently, day and night, guiding me through whenever needed.

I whole heartedly thank Dr. Kinzy Jones Jr., for guiding me through the process of FEA analysis, without which this wouldn't have been possible. His guidance has helped me save more than 6 months of training in Hypermesh and Abaqus. To be honest it would have taken longer than 6 months to get to this speed.

Dr. Marc Zampino for giving me sufficient feedback and provide brief outline on how to start and write a dissertation. Dr George Florence for providing me some insight on statistical analysis. 
We would like to acknowledge the Instructional \& Research Computing Center (IRCC) at Florida International University for providing HPC software Abaqus and Matlab that contributed to part of the research results within this document. I specially thank both Dr. Mengxing Cheng and Dr. Cassian D’Cunha from IRCC to provide needed support and training to get used to the High Performance Computing interface.

I would like to thank my group member, Sadegh Behdad to help me out whenever I needed help in every aspect including course work, research or personal help.

I would like to thank my friends, Mr. Subrahmanyam V.V, Dr. Aparna Aravelli, Dr. Ravi K. Gudavalli for providing their valuable inputs in my research and document writing and editing. I especially would like to thank my college friends, Subrahmanyam and Aparna, for their valuable time and support to discuss and guide me in difficulties I faced in research or elsewhere. I not only learned science but life lessons from them.

I would like to thank all my family members, especially my wife, parents, brother, sister, cousins, my in-laws and all my near and dear relatives. You are my biggest strength in life, without your unconditional love and support this day would not have come. My wife, played a big supporting role in helping me during this $\mathrm{PhD}$.

I would like to acknowledge the Department of Mechanical and Materials Engineering for supporting me through graduate and teaching assistantship awards.

I would like to thank all of you whose names I forgot to write, but learned important lessons from you. 


\title{
ABSTRACT OF THE DISSERTATION
}

\section{A NOVEL HIP IMPLANT USING 3D WOVEN COMPOSITE MATERIAL - DESIGN}

AND ANALYSIS

by

\author{
Hari Kishore Adluru
}

Florida International University 2015

Miami, Florida

\section{Professor Benjamin Boesl, Major Professor}

The present research focuses on analyzing the possibility of implementing three dimensional woven composite (3DWC) materials in hip implants. The integration of 3DWCs in hip implants has the possibility to both extend the life-time and improve patient outcomes; by spatially varying mechanical properties to meet both biological needs as well as required mechanical loading. In this study, the bulk material properties of 3DWCs were varied based on woven composite architecture and determined using physics based models, which reflect the realistic geometries of fibers in compaction and preform. The multi-digital chain method combined with Extended Finite Elemental Analysis (XFEA) are adopted in this micro-analysis for composite design. Four different woven architectures with a combination of different existing biocompatible fiber and resins are considered in this study. The main objective is to assess the mechanical response of these biocompatible materials in the design of 3D woven architectures and determine their ability to match the required modulus at different regions of a hip implant. Results obtained show 3DWCs are viable candidates for this application. Multiple architectures and materials chosen, were able to achieve the desired mechanical 
response. Additional studies can use these results as a starting point and framework for further mechanical and biological testing. 


\section{TABLE OF CONTENTS}

CHAPTER

PAGE

CHAPTER 1- Introduction ......................................................................... 1

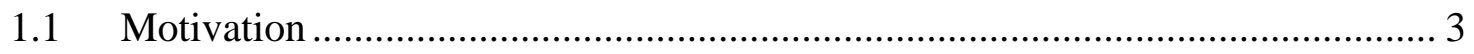

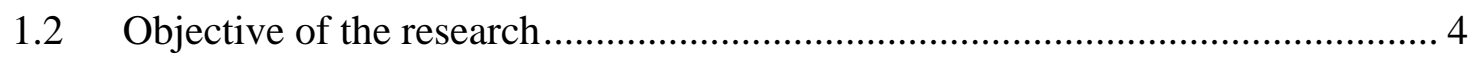

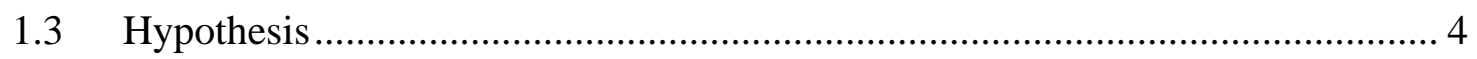

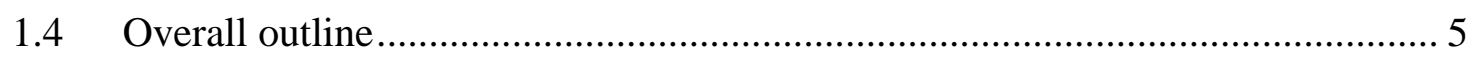

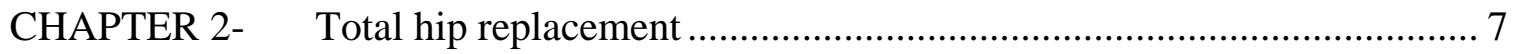

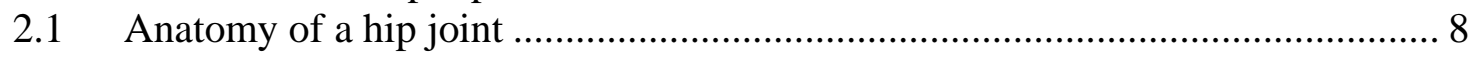

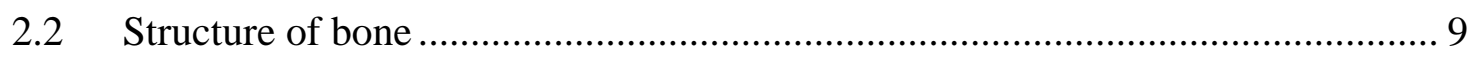

2.3 Hip joint forces and range of motion .......................................................... 11

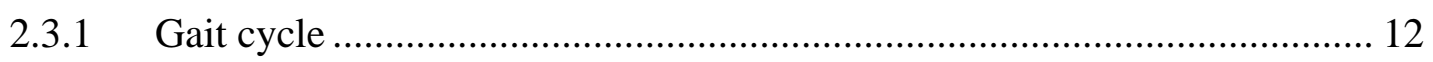

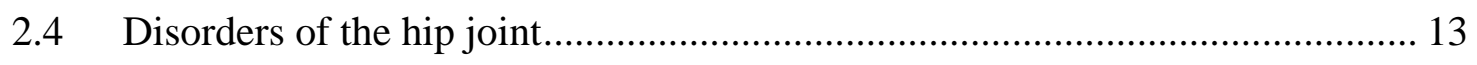

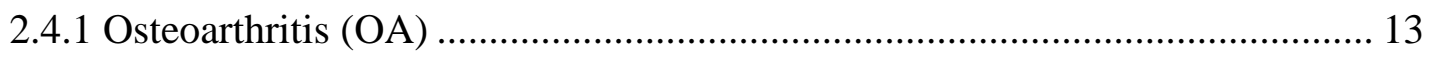

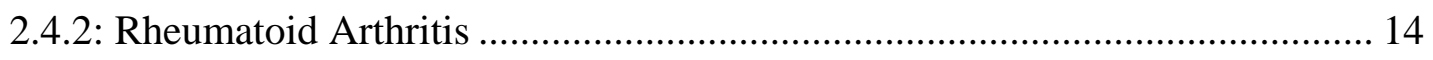

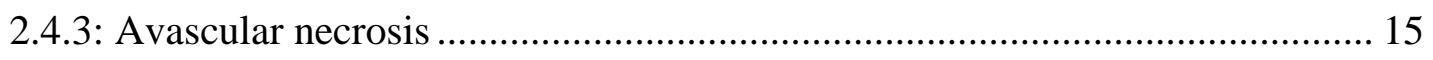

2.4.4: Non-operative management of OA and need for THR ................................ 16

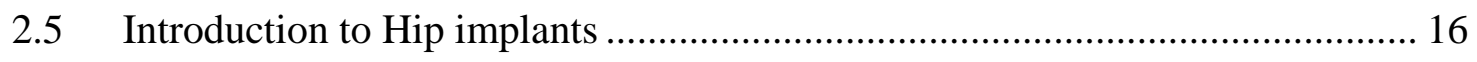

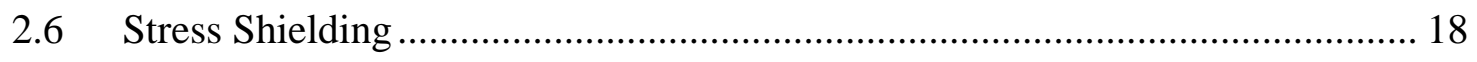

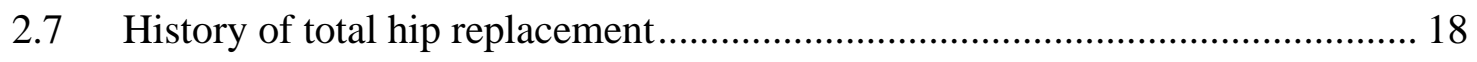

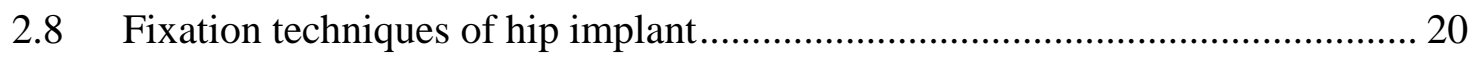

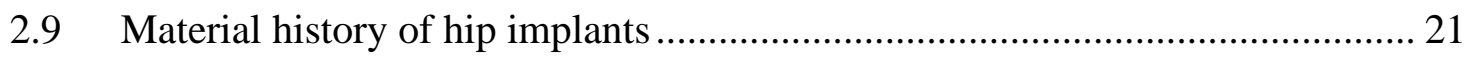

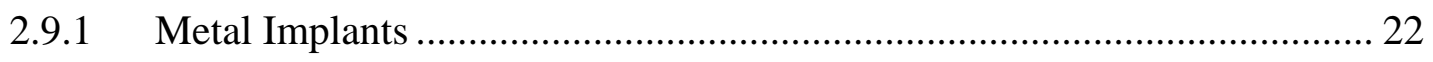

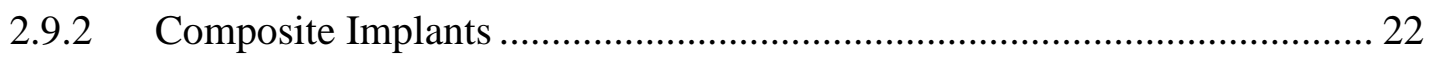

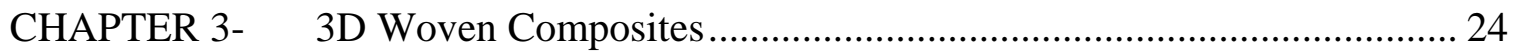

3.1 Introduction on composite material design ................................................. 24

3.2 Transverse Stiffnes -Elastic Moduli Ey and $\mathrm{E}_{z}$.......................................... 27

3.3 Additional approaches in the design of composite materials ........................... 29

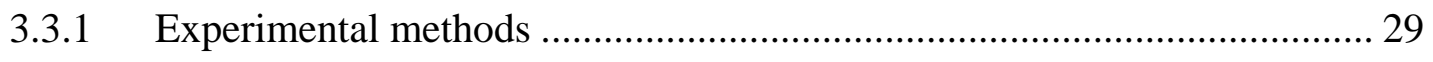

3.3.2 Finite Element Analysis (FEA) ........................................................ 29 
CHAPTER 4- Design of 3DWCs using VTMS ...................................................... 36

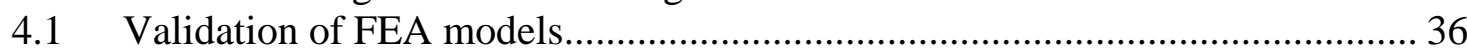

4.1.1 Finite Element based Hexagonal Unit Composite Cell analysis ................. 36

4.1.2 Finite Element Analysis of 3D Orthogonal Woven Composites ................. 38

4.1.3 Model Validation and Comparison of present Results with Literature ...... 42

4.2 Extended Finite Element based Modeling ………........................................ 45

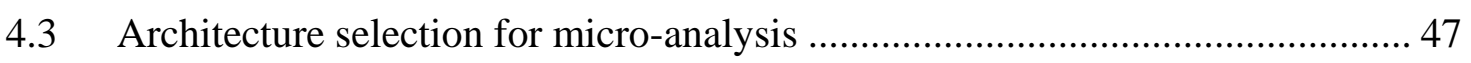

4.3.1 Development of 3D Orthogonal Woven Structure (3DOW) ...................... 48

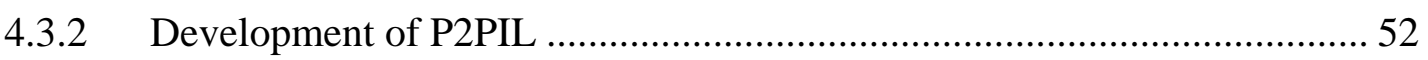

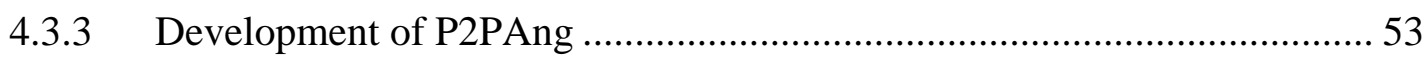

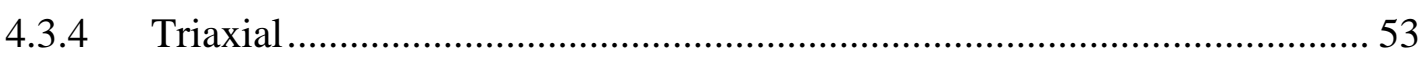

4.4 Material Selection for the micro-analysis .................................................... 54

CHAPTER 5- Finite Element Analysis of Hip Implant.............................................. 56

5.1 Development of solid hip implant model....................................................... 56

5.2 Preparation for solid processing into HyperMesh.............................................. 58

5.2.1 Preprocessing of finite element model...................................................... 58

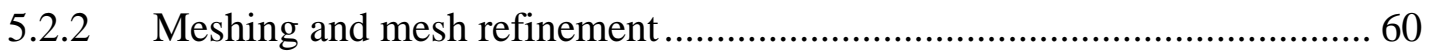

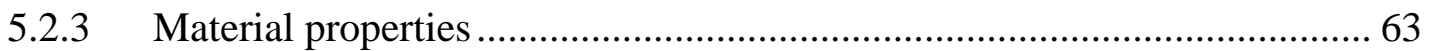

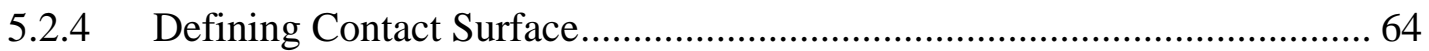

5.2.5 Boundary and load conditions ................................................................. 64

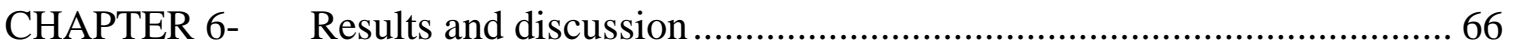

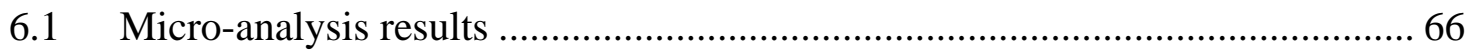

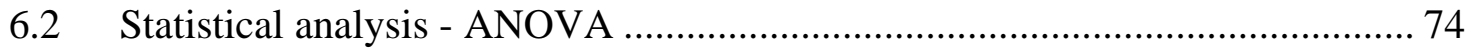

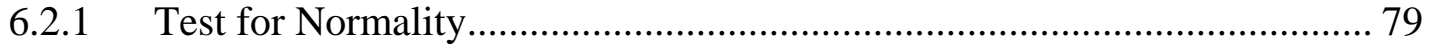

6.3 FEA results of hip implants..................................................................... 79

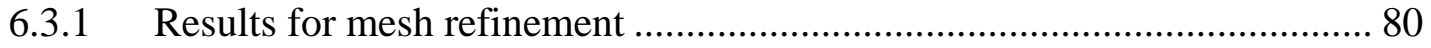

6.3.2 Comparison of Implants models .............................................................. 84 
CHAPTER 7- Conclusions and future work....................................................... 88

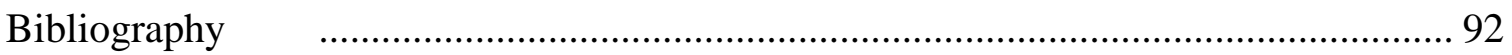

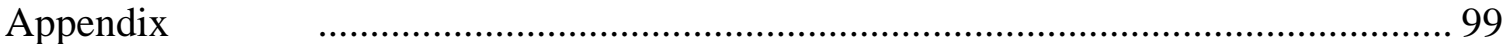

VITA 


\section{LIST OF TABLES}

TABLE

PAGE

Table 1 Prevalence of radiographic osteoarthritis in the hip [28]................................ 14

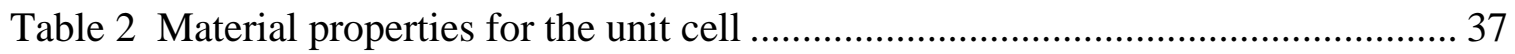

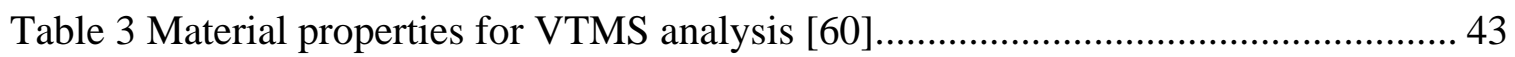

Table 4 Mechanical Properties of fibers and resin................................................... 54

Table 5 Details of different implant models ........................................................... 59

Table 6 Implant 1 with target element size, number of nodes and elements ................... 62

Table 7 Implant 2 with target element size, number of nodes and elements ................... 62

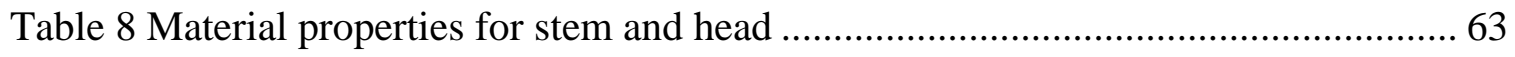

Table 9 3D Orthogonal Woven Composites 6\% Z yarns - elastic modulus (GPa) longitudinal

Table 10 Ply to Ply Interlocking without Warp - elastic modulus (GPa) Longitudinal... 69

Table 11 Ply to Ply Interlocking without Warp - elastic modulus (GPa) Transverse ...... 70

Table 12 Ply to Ply Angle Interlocking without Warp - elastic modulus (GPa)

Longitudinal.

Table 13 Ply to Ply Angle Interlocking without Warp - elastic modulus (GPa)

Transverse 71

Table 14 Tri-axial - elastic modulus (GPa) Longitudinal .......................................... 73

Table 15 Tri-axial - elastic modulus (GPa) Transverse ............................................. 73

Table 16 Independent factors considered for ANOVA …......................................... 75 


\section{LIST OF FIGURES}

FIGURE

PAGE

Figure 1 Total hip replacement with a metal prosthesis ............................................. 1

Figure 2 Schematic of Stress Shielding [7] .......................................................... 2

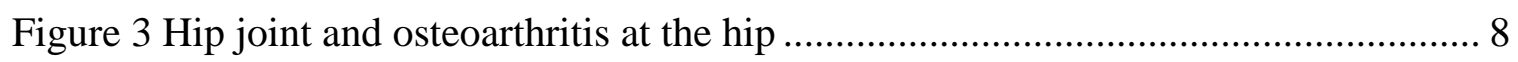

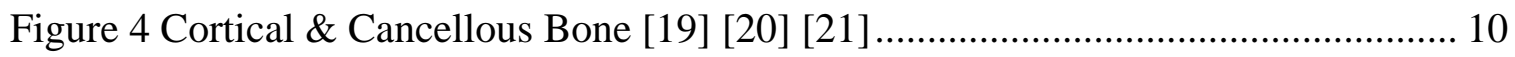

Figure 5 Femur Bone Design and Stress [23] ...................................................... 11

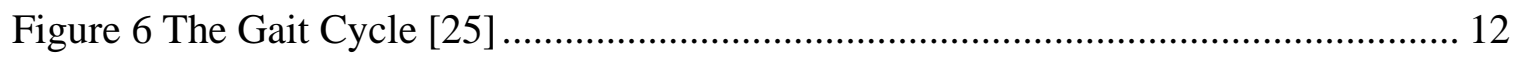

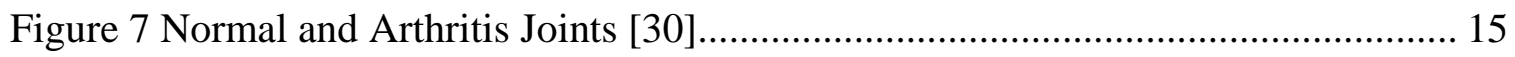

Figure 8 Diaphragm and radiograph of a hip replacement [44] ................................. 17

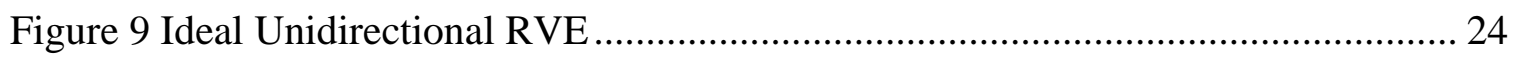

Figure 10 Efficiency Krenchel Factor Diagram.................................................... 25

Figure 11 Approximation using 1D cubic B-splines .............................................. 33

Figure 12 Hexagonal Unit Composite Cell................................................................. 36

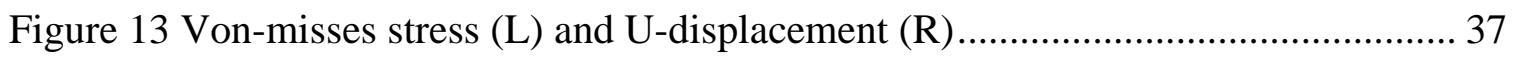

Figure 14 Stress vs Strain plots for different volume percent ..................................... 38

Figure 15 Predefined 3D Orthogonal Fibers a) Isometric View b) Front View c) Top

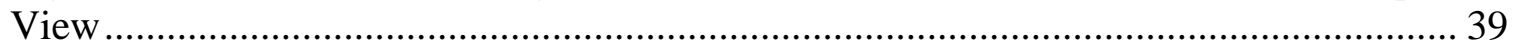

Figure 16 3D Orthogonal tows after compaction a) Isometric View b) Top View .......... 40

Figure 17 Matrix and fibers showing Boundary Conditions .................................... 40

Figure 18 U-displacement of the 3DW Composite RVE........................................... 41

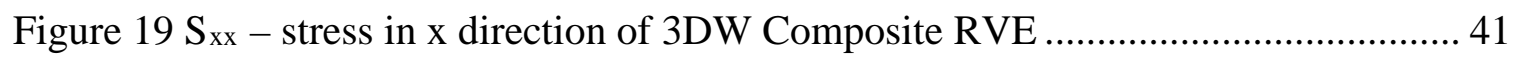

Figure 20 Stress-strain graph for $6 \% \mathrm{Z}$ yarns at $0^{\circ}$ orientation of the fibers ................... 42 
Figure 21 Stress-strain graph for $10 \% \mathrm{Z}$ yarns at $0^{\circ}$ orientation of the fibers ................. 42

Figure 22 Stress-strain graph for $6 \% \mathrm{Z}$ yarns at $0^{\circ}$ orientation of the fibers ................... 44

Figure 23 Stress-strain graph for $10 \% \mathrm{Z}$ yarns at $0^{\circ}$ orientation of the fibers ................. 45

Figure 24 General steps involved in developing 3D Woven Composite Model ............. 47

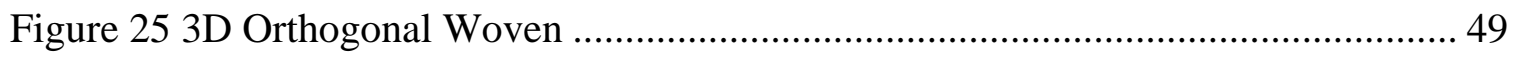

Figure 26- a) 3DOWC Meshed model with Boundary Conditions b) P2PILwoW showing Interface elements - connection of tow and resin elements............................ 51

Figure 27 Ply to Ply Inter- Lock without Warp ...................................................... 52

Figure 28 Ply to Ply Angle Inter- Lock without Warp ............................................... 53

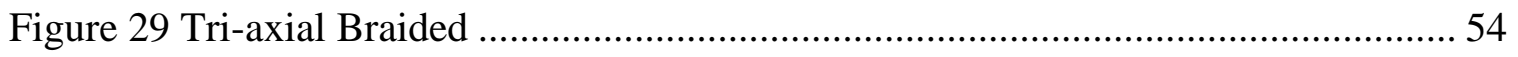

Figure 30 Stem dimensions a) lengthwise b) cross-section profiles [75] ...................... 57

Figure 31 (a) Assembly of Hip Implant in SolidWorks (b) Finite Element Mesh ........... 58

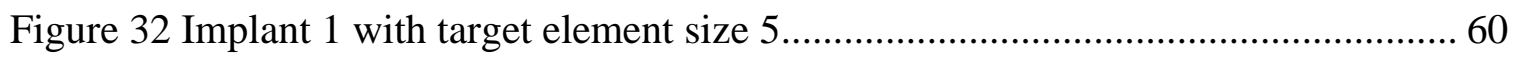

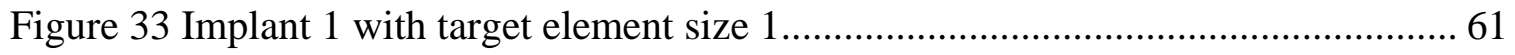

Figure 34 Young’s modulus for base model and functionally graded model w.r.t length 63

Figure 35 Stem showing functionally graded material sections. ................................. 64

Figure 36 Implant showing boundary and loading conditions.................................... 65

Figure 37 Stress Sxx of Ortho model - Z6\% loaded in X direction a) Unit Composite

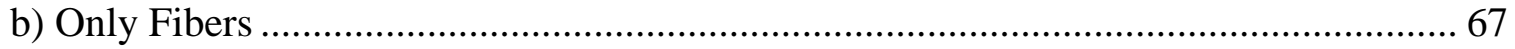

Figure 38 Stress Szz of P2PIL loaded in Z direction (a) Unit Composite (b) Fibers

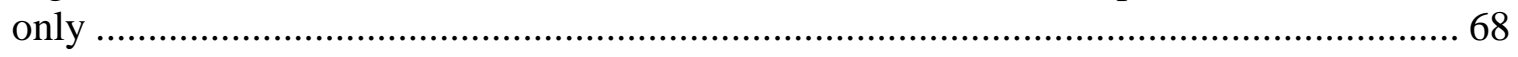

Figure 39 Stress-strain plots for P2PIL with PEEK as resin. ..................................... 69

Figure 40 Stress Szz of P2PAng loaded in Z direction (a) Unit Composite (b) Fibers

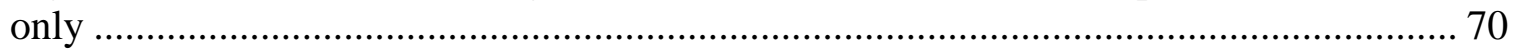


Figure 41 Stress Sxx of Traxial Braid loaded in X direction (a) Unit Composite (b)

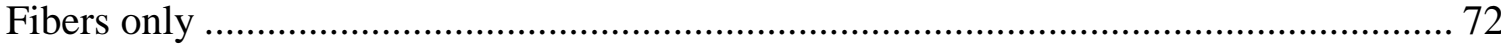

Figure 42 Elastic Modulus for different architectures and fibers with PEEK as resin ..... 74

Figure 43 Estimated marginal mean elastic modulus (GPa) in longitudinal direction ..... 77

Figure 44 Estimated marginal mean elastic modulus (GPa) in transverse direction ........ 77

Figure 45 Box plot showing mean and variance of elastic modulus w.r.t fibers .............. 78

Figure 46 Box plot showing mean and variance of elastic modulus w.r.t matrix............ 78

Figure 47 Residual plots for elastic modulus...................................................... 79

Figure 48 Implant 1 -Displacement (in mm) using different mesh sizes ....................... 80

Figure 49 Implant 1 - von Mises Stress (in MPa) using different mesh sizes................. 81

Figure 50 Path1 on surface of stem for displacement and stress plots .......................... 82

Figure 51 Implant1- displacement along the surface path with different mesh sizes ....... 83

Figure 52 Implant1- displacement near centerline with different mesh sizes ................. 83

Figure 53 Displacement plot on Implant 1 and 3 on surface ......................................... 85

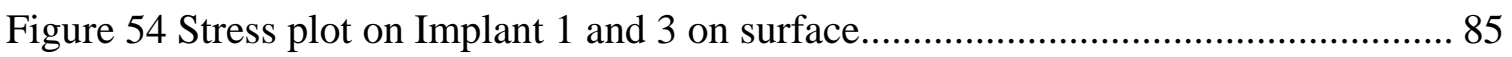

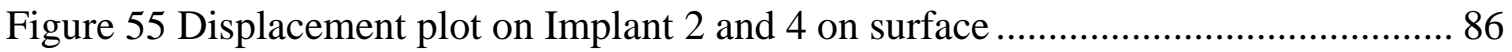

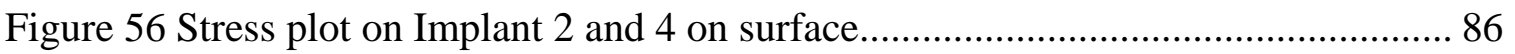

Figure 57 Functionally graded material properties for different $n$ values ...................... 90 


\section{ACRONYMS}

THR

TKR

OA

NSAID

COX-2

HA

PMMA

UHMWPE

PTFE

MOM

COC

$\mathrm{COM}$

ROM

VTMS

BSAM

FEA

3DWC

RTM

RVE

3DOWC

P2PIL

P2PAng
Total Hip Replacement

Total Knee Replacement

Osteoarthritis

Non-steroidal anti-inflammatory drug

Cyclooxygenase-2

Hydroxyapatite

Poly-methyl methacrylate

Ultra-High Molecular weight Polyethylene

Polytetrafluoroethylene

Metal-on-metal

Ceramic-on-ceramic

Ceramic-on-metal

Rule of mixtures

Virtual Textile Morphology Suite

B-Spline Analysis Method

Finite Element Analysis

3D Woven Composite

Resin Transfer Mold

Representative Volume Element

3D Orthogonal Woven Composites

Ply to Ply Inter-Lock without warp

Ply to Ply Angle Inter-Lock without warp 


\section{NOMENCLATURE}

\begin{tabular}{|c|c|}
\hline$V_{f}$ & Fiber volume fraction \\
\hline$V_{m}$ & Matrix volume fraction \\
\hline$\rho_{f}$ & Fiber density \\
\hline$\rho_{m}$ & Matrix density \\
\hline$W_{f}$ & Fiber weight \\
\hline$W_{m}$ & Matrix weight \\
\hline$E_{f}$ & Young's Modulus of the fibers \\
\hline$E_{m}$ & Young's Modulus of the matrix \\
\hline$E_{c}$ & Young's Modulus of the composite \\
\hline$E_{11}$ & Longitudinal stiffness \\
\hline$E_{22}$ & Transverse stiffness \\
\hline$G_{12}$ & In-plane shear Modulus \\
\hline$\vartheta_{12}$ & Poisson’s ratio \\
\hline$\epsilon$ & Axial/normal strain \\
\hline$\gamma$ & Shear strain \\
\hline$\sigma$ & Normal stress \\
\hline$\tau$ & Shear stress \\
\hline$N_{i}$ & Shape function where ' $i$ ' is the number of nodes \\
\hline
\end{tabular}




\section{A NOVEL HIP IMPLANT USING 3D WOVEN COMPOSITE MATERIAL - DESIGN}

AND ANALYSIS

\section{CHAPTER 1-Introduction}

Total Hip Replacement (THR) surgery has become one of the most common orthopedic procedures due to several medical conditions, such as arthritis, fracture or dislocation due to trauma on the hip joint. After Total Knee Replacement (TKR) surgery, THRs are the most common joint replacement surgeries with more than 150,000 performed a year in the USA alone [1].

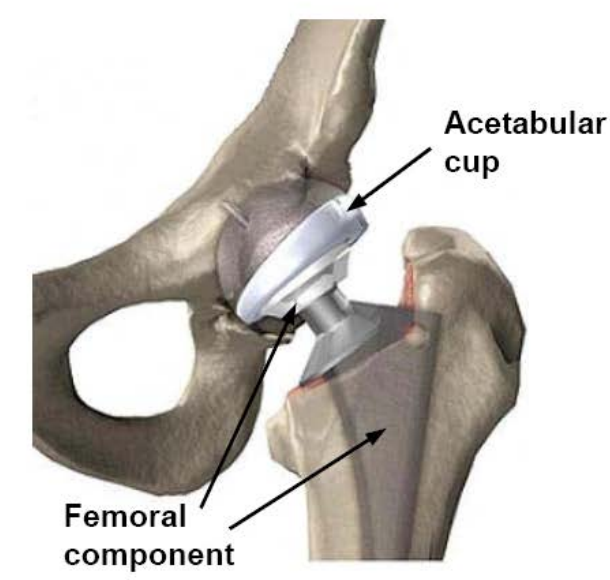

Figure 1 Total hip replacement with a metal prosthesis

Hip implants work as a ball and socket joint shown in Figure 1 and can be made of metal and plastic, metal on metal, ceramic on ceramic and metal with highly cross linked polyethylene. Metal on metal hip implants are still predominantly used due to their very low wear rate and longer life. The clinical success rate of metal hip implants is 93\% in 10 years and $85 \%$ in 15 years post-surgery [2]. The drawbacks of the metallic hip implants 
include release of metal ions into the bloodstream, which increases the risk of cancer and other diseases.

One of the major concerns in hip implant failure is stress shielding. Clinical studies and literature from the past two decades suggested that there is mismatch of stiffness between the implant and the femur bone resorption, which causes bone softening [3-5], as shown in Figure 2. This contributes to the long-term implant failure called stress shielding. Previous studies [6] using unidirectional composite materials have shown the potential to eliminate the stress shielding effect, but the drawbacks include lack of required modulus and strength in critical regions. In addition, transverse properties were observed to be low and de-lamination and micro cracking initiation started at relatively low loading values. The failure of metal hip implants due to stress shielding and the drawback of de-lamination in unidirectional composite material implants, indicated the need and scope for improvement in the design of hip implants.

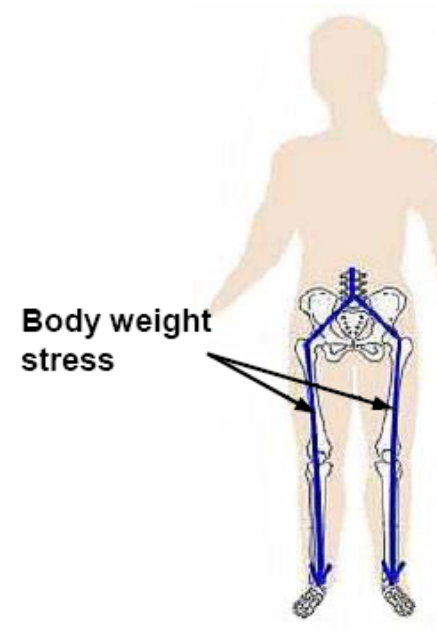

(a)

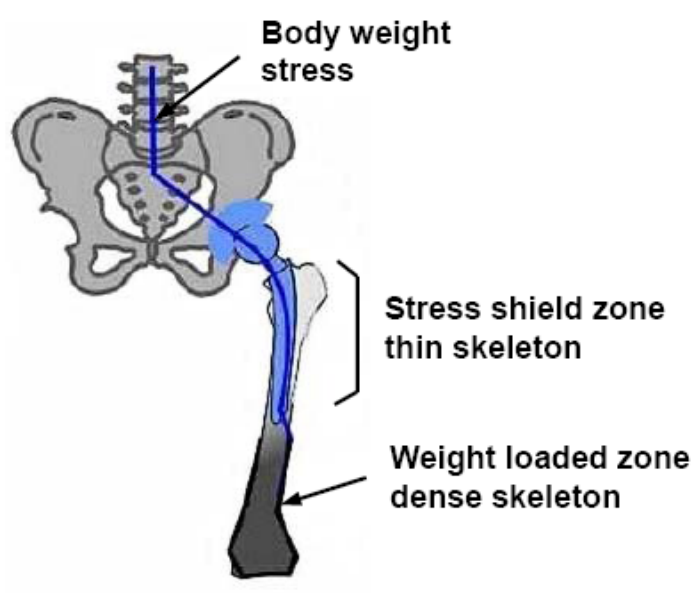

(b)

Figure 2 Schematic of Stress Shielding [7] 
3DWC structures offer several advantages, which include tailored and functionally graded mechanical properties. These functionally graded properties are achieved by systematically changing the fiber to matrix volume ratios, using different weave patterns - orthogonal woven, ply-to-ply interlocking and angle interlocking. These parameters help a designer to vary modulus and strength properties of the implant to the desired value at distal and proximal end of the hip joint. Literature indicates that extensive experimental and computational work is performed to determine the mechanical properties of fiber reinforced woven composites [8]. Micro-mechanics of 3DWCs have been studied for the past two decades. Since micro-mechanics is highly challenging and includes the need for high computational power, some researchers have developed the digital chain and multi-digital chain element approach to represent yarn of fibers [9]. Several researchers have adopted these techniques to determine the properties of different architectures in 3DWCs. Some computational results were verified with experimental results using these multi-digital chain approach's [10-13]. Finite elemental analysis (FEA)-based studies and analysis are widely used and accepted in the biomedical field, including simulations of hip joints. These studies drastically reduce the number of experiments on the human and animal subjects.

\subsection{Motivation}

Hip implants are made of several materials and each one has its own benefits and limitations. In recent years, doctors saw an increase in revision surgeries of THRs due to their high failure rates. Medical experts believe these revised surgeries are more tedious to perform, recovery time is more for the patient, success rate is low and it also has financial burden on medical facilities. After reviewing the major reasons for failure of the 
hip implant, it motivated us to design and analyze a novel composite material for hip implants.

\subsection{Objective of the research}

The main objective of the present research is to determine the bulk material properties of 3DWCs for use in biomedical applications, especially in the design of hip implants. Finite element-based micro-analysis is performed on different architectures of the 3DWCs. Micro-analysis is executed to determine the bulk material properties of several 3D architectures, choosing four suitable architectures. Once proven feasible, these material properties are considered in the macro-analysis of the hip joint to determine the stress distribution on the hip implant considering normal loading conditions based on the gait cycle.

\subsection{Hypothesis}

Question \# 1: What is the influence of woven composite architecture and constituents on bulk material properties?

Hypothesis 1: Systematically changing the architecture and constituent properties, the overall bulk material properties of 3DWCs can be tailored to meet desired specifications. Adaption of a special technique called multi-chain digital element (in VTMS) to represent yarn of fibers in a 3DWC will result in accurate prediction of the bulk material properties of the composite within an acceptable margin of error.

Question \# 2: Will the proposed redesign reduce failures of hip implants caused due to stress shielding?

Hypothesis 2: When the redesigned hip implant is subjected to mechanical loading, the proposed designs will significantly transfer the loading to the bone, thus avoiding the 
stress shielding caused by bone softening. The modulus of the 3DWC material at the stem (region) is to match the properties of the bone and is to be higher at the neck region. Targeted material specification at the distal end should be between 40 to 95 GPa in loading direction and 15 to 30 GPa in non-loading direction [14].

Question \# 3: Will the proposed functionally graded composite material in the stem of hip implant cause changes in stress distribution?

Hypothesis 3: Determine the baseline stress distribution in the hip implant using regular materials and then compare the same with functionally graded material properties in the stem of hip implant. If stress is distributed over the length of the stem instead of concentrating on the neck, it can be concluded that this will minimize stress shielding.

\subsection{Overall outline}

Chapter 1 provides comprehensive review of the present research work, followed by stating the objectives of the present work. Finally, the hypothesis is presented.

Chapter 2 introduces the basic anatomy of hip joints and discusses the need and reasons for THR. A brief history of the hip implants used in the past and present is given and finally the materials used for hip implants are discussed. The benefits and limitations of each of those are presented.

Chapter 3 presents the various analytical formulations available for design of composites, limitations and other approaches in designing composites. The methods used to determine the bulk material properties of 3D Woven composites is also presented in this chapter. 
In Chapter 4, validation of micro-mechanics models, XFEA method, its advantages, micro-analysis of 3D woven architectures, and brief discussion of the results obtained from the micro analysis of the 3DWCs is presented.

Chapter 5 deals with finite element analysis of hip implant making use of the results obtained from micro-analysis. Development of solid hip implant model, meshing, materials and boundary conditions considered are discussed.

In Chapter 6, the results from both analysis are discussed in detail. Summary of the results is discussed and conclusions are made of the present work. This is followed by the recommendations for future research. 


\section{CHAPTER 2- Total hip replacement}

Several medical conditions, such as osteoarthritis, rheumatoid arthritis, avascular necrosis, fracture or dislocation due to trauma to the hip joint, lead to chronic hip pain and disability, which require THR. Arthritis is one of the major causes requiring hip replacement surgery. Arthritis affects more than 40 million Americans, more than 10 million in the United Kingdom and tens of millions worldwide. Metallic hip implants, more specifically Ti based alloys are still predominantly used for the stem of hip implant. The clinical success rates for metal hip implants are 93\% for 10 years and 85\% for 15 years post-surgery [2]. A brief description of the background for hip implants is given next.

THR dramatically improves the quality of life of patients suffering from debilitating hip disorders. This procedure can bring almost immediate relief to the unremitting pain, due to which the functional capacity of the lower limb has been greatly decreased. Especially to those patients with an end-stage degenerative joint disease, THR is often the final attempt that the surgeons resort to in terms of pain relief and increasing mobility [15].

Before discussing details of hip replacements and hip implants, it is good to know the anatomy of a hip joint along with the forces acting on the joint due to human motion. This section gives a brief description of a hip joint. The causes of hip malfunctioning are detailed and the remedies considered are discussed. History of the materials used in hip implants and hip replacement techniques are also explained. 


\subsection{Anatomy of a hip joint}

A better understanding of the hip joint and the conditions, which cause its malfunctioning are needed before designing a hip implant. In this section, the anatomy of a hip joint is discussed. The hip joint shown in Figure 3 is a synovial joint that connects the lower limb to the trunk of the human body. It consists of a synovial ball (femoral head) and a wide range of moments [16]. The hip joint serves a very important biomechanical function in the human body. While supporting the majority of the human body ( $70 \%$ of the total body weight), the joint simultaneously provides stability, especially during the movement of the trunk on the femur, during walking and running. The stability of a hip joint is determined by the shape of the articular surface, the strength of the joint capsule, ligaments and the muscles.

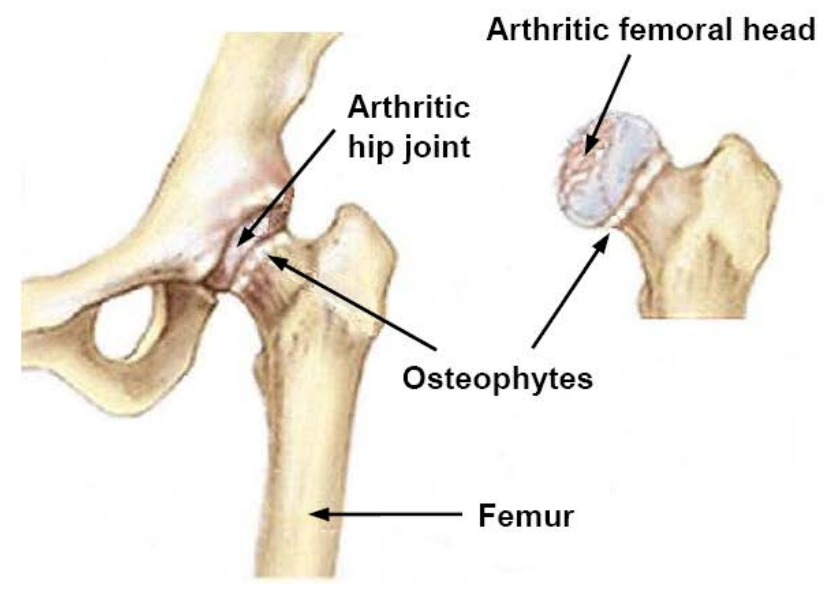

Figure 3 Hip joint and osteoarthritis at the hip

The contact area between the femoral head and the acetabulum is limited at low loading conditions. The contact area increases with increasing loads, thus distributing the load over greater area and as a result, reducing excessive stress in the underlying 
cartilage. The contact stresses are higher at the superior surfaces of the femoral head and acetabulum compared to other parts of the articulating surface. This difference in stress distribution is due to the orientation of the femur relative to the pelvis. Therefore, the articular cartilage is thicker in the superior region than elsewhere.

The angle between the femoral neck and the shaft is called the angle of inclination in the frontal plane and the angle of ante-version in the horizontal plane. The angle of inclination of the femoral head in an average adult is around $125^{\circ}$ and the angle of ante-version is around $10^{\circ}$. These angles play an important role in the stability of the hip joint [16].

\subsection{Structure of bone}

Bone is composed of collagen, water, a mineral called hydroxyapatite (HA), proteoglycans and non-collagenous protein [17]. Collagen, a structural protein, gives bone flexibility and tensile strength, while also providing loci for nucleation of bone mineral crystals. Up to $50 \%$ of bone weight is comprised of the bone mineral hydroxyapatite $\mathrm{Ca}_{10}\left(\mathrm{PO}_{4}\right)_{6}(\mathrm{OH})_{2}$ - imparting bone with rigidity and compressive strength [18]. The specific role of proteoglycans and non-collagenous protein is not entirely clear and may function to control the location or rate of mineralization in bone. The water content in bone is approximately $60 \%$ by volume and is present, both as free water or bonded with other molecules. While in principle the porosity of bone can vary continuously from 0 to $100 \%$, in fact most bone tissues are of either very low or very high porosity, with little bone of intermediate porosity.

These two types of bone, shown in Figure 4, correspond to the cortical bone and cancellous bone, respectively [19-21]. 


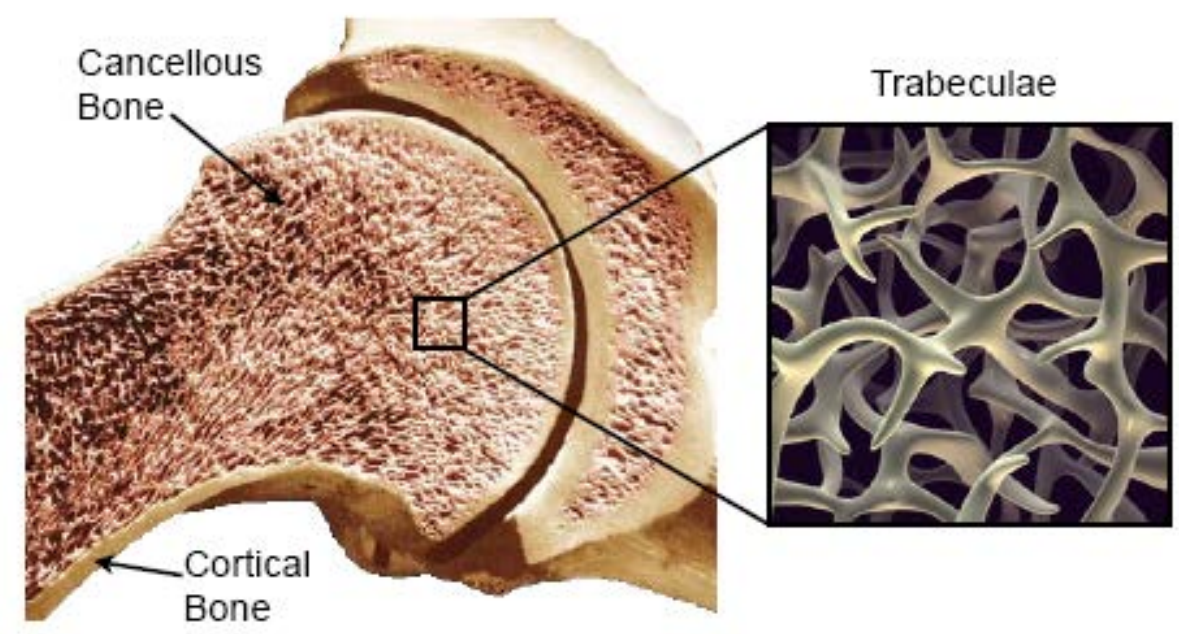

Figure 4 Cortical \& Cancellous Bone [19-21]

Cortical bone, as its name implies, forms the cortex or outer most shell, of most bones. Compared to cancellous bone, cortical bone is much denser, with a typical porosity of 5 to $10 \%$. Cortical bone, which is also known as a compact bone, is much harder, stiffer and stronger than cancellous bone [17] [21] [22]. The thickness of cortical bone in the pelvis ranges from $0.5 \mathrm{~mm}$ to $3 \mathrm{~mm}$, whereas in the femur bone, the thickness ranges from $0.5 \mathrm{~mm}$ to over $10 \mathrm{~mm}$ in the shaft.

Cancellous bone, synonymous with trabecular or spongy bone, is porous bone found in the ends of long bones, proximal to the joints. The porosity of cancellous bone is between $75 \%$ and $95 \%$. Cancellous bone is highly vascular and is composed of struts called trabeculae, each approximately $200(\mu \mathrm{m})$ in thickness, as shown in Figure 4. At times the trabeculae appear to be organized to orthogonal arrays; often they are more randomly arranged. In contrast to cortical bone, cancellous bone is much more flexible, softer and less dense [17] [21] [22]. 


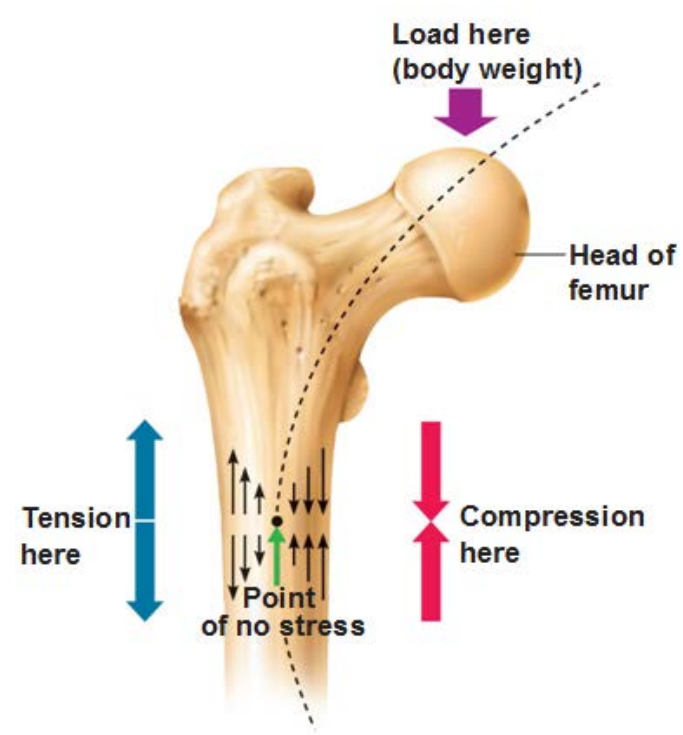

Figure 5 Femur Bone Design and Stress [23]

In addition to knowing the anatomy of the bone, the main feature required for modeling a hip implant is knowledge of various forces acting on the hip joint. These forces lead to its proper functioning and reliability. This aspect is explained in the following section.

\subsection{Hip joint forces and range of motion}

Human motion results in different types of forces acting on the hip joint. The forces on the hip joint are estimated to range from 1.5 times to 5 times the weight of the human body during normal gait cycle. The hip joint forces are maximal at the heel strike and toe off and minimal during the swing phase [24]. The hip joint allows movement in three planes. The largest range of motion of $165^{\circ}\left(20^{\circ}\right.$ extension and $145^{\circ}$ flexion) in the hip joint occurs in the sagittal plane. The range of motion is around $65^{\circ}$ in the frontal plane and the medial and lateral rotations are of range $70^{\circ}$ and $90^{\circ}$, respectively, in the transverse plane. A more systematic representation of the forces acting on the hip joint due to the motion of the leg, also called the gait cycle, is described below. 


\subsubsection{Gait cycle}

The gait cycle can be divided into eight events, five during the stance phase and three during the swing phase. The names of these events are self-descriptive and are based on the movement of the foot, as shown in Figure 6.

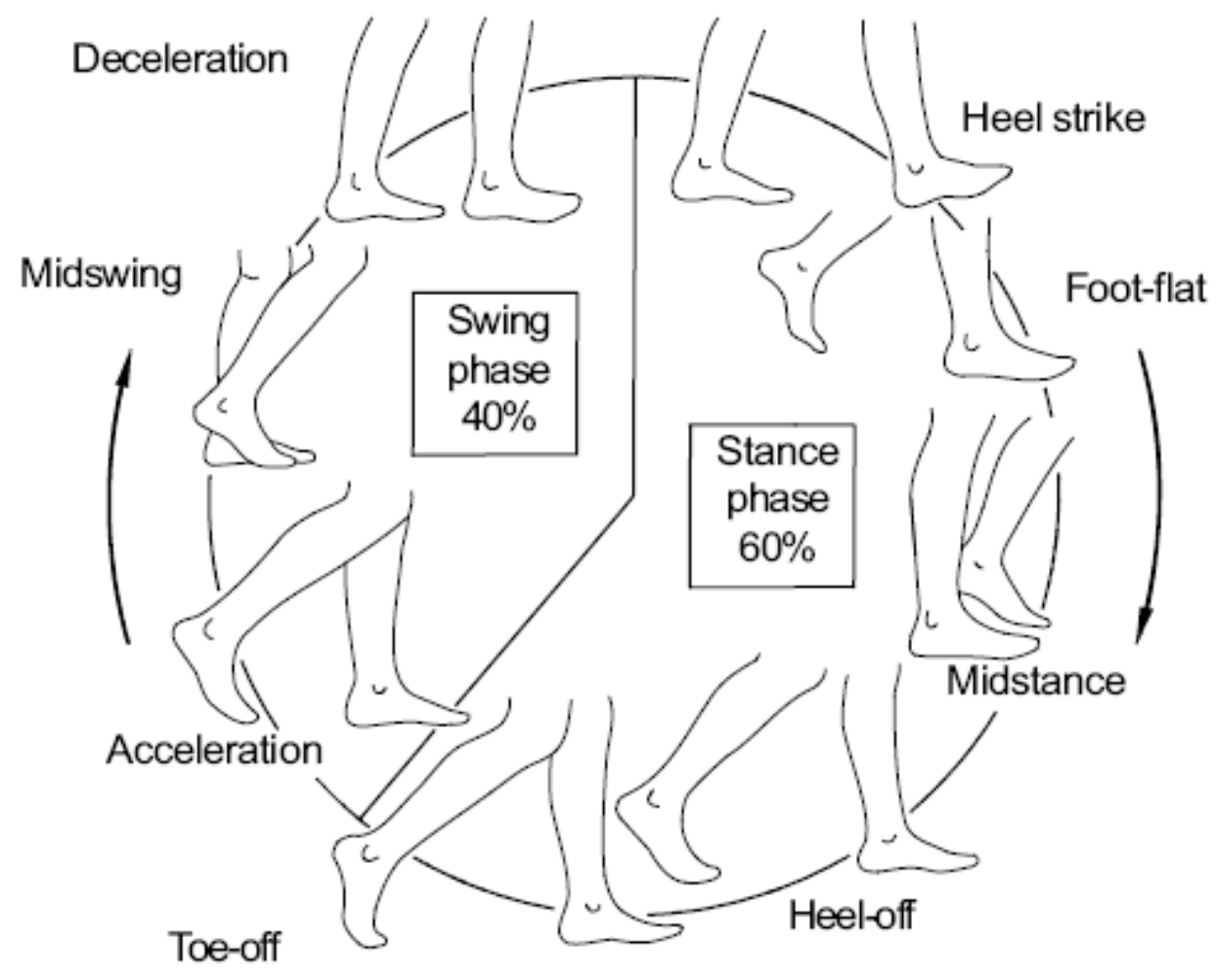

Figure 6 The Gait Cycle [25]

In the traditional nomenclature, the stance phase events are as follows [25] [26].

Heel Strike initiates the gait cycle and represents the point at which the body's center of gravity is at its lowest position;

Foot - flat takes place when the sole of the foot touches the ground; 
Mid-stance occurs when the swinging foot passes the stance foot and the body's center of gravity is at its highest position;

Heel off occurs as the heel loses contact with the ground;

Toe-off terminates the stance phase as the foot leaves the ground.

The swing phase events are as follows:

Acceleration begins as soon as the foot leaves the ground and the subject activates the hip flexor muscles to accelerate the leg forward;

Mid-swing occurs when the foot passes directly beneath the body, coincidental with midstance for the other foot;

Deceleration describes the action of the muscles as they slow the leg and stabilize the foot in preparation for the next heel strike.

\subsection{Disorders of the hip joint}

Over time the forces acting on the hip joint due to gait cycle and some other traumatic events cause the hip joint to malfunction and lead to disability and severe pain. A description of three main types of hip joint disorders is given in this section.

\subsubsection{Osteoarthritis (OA)}

Osteoarthritis occurs when the protective cartilage, especially in weight bearing joints, such as hips, knees, spine, etc., wears down over time. The breakdown of cartilage tissue is dependent upon loss of the amorphous portion of the matrix and the collagen framework, which results in erosion or lesions, leading to degeneration of articular cartilage lining. The breakdown of this tissue leads to bones rubbing against each other causing, severe pain [27]. This disorder mainly effects older people and can range from mild to severe conditions. It is not only age-related but can also occur due to high levels 
of stresses in the joint due to extensive sport activities. There is also a high correlation with obesity. Table 1 shows the prevalence of radiographic osteoarthritis in the hip joints of both sexes in different age groups.

Table 1 Prevalence of radiographic osteoarthritis in the hip [28]

\begin{tabular}{ccc}
\hline Age & Sex & Prevalence \\
\hline$<55$ & Men & $1 \%$ \\
& Women & $1 \%$ \\
\hline $55-65$ & Men & $3 \%$ \\
& Women & $2 \%$ \\
\hline$>65$ & Men & $6 \%$ \\
& Women & $4 \%$ \\
\hline
\end{tabular}

\subsection{2: Rheumatoid Arthritis}

Rheumatoid arthritis is a systemic inflammatory disorder that often progresses to cause destruction of the articular cartilage. The initial cause of this disease is not well known, however immunity plays a vital role in its progression and chronicity. It usually initiates as inflammation in the synovium, causing thickening of the membrane and proliferation of the synovial fluid and the connective tissue. This will lead to the loss of stability, thinning of the articular cartilage and destruction of the joint [27] [29]. 


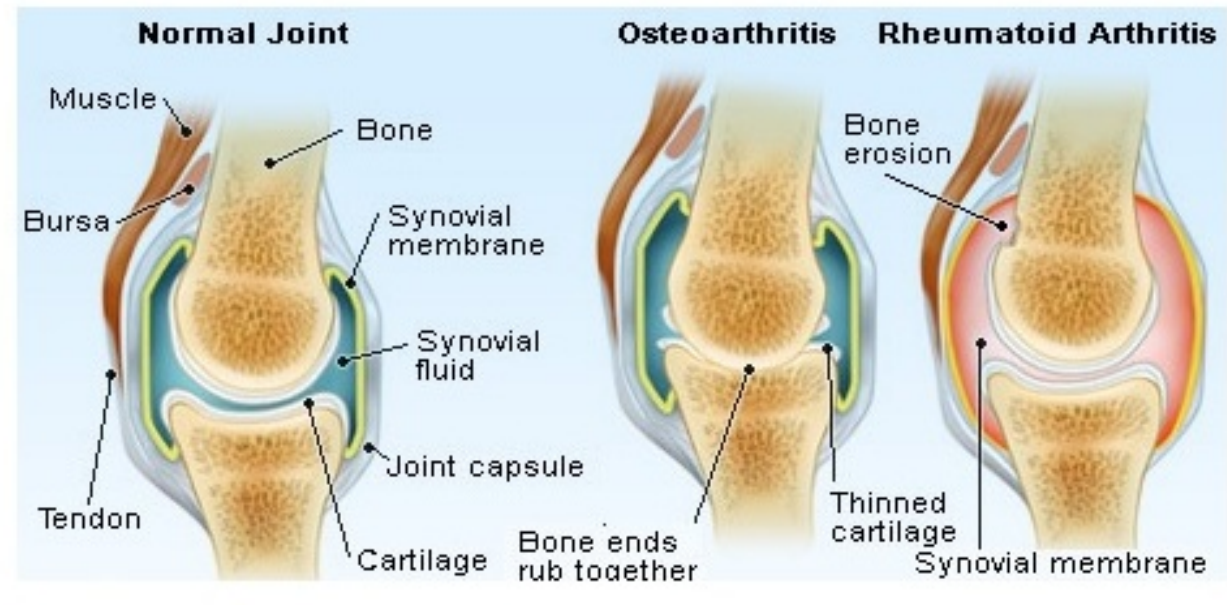

Arthritic Joints

Figure 7 Normal and Arthritis Joints [30]

\subsection{3: Avascular necrosis}

Avascular necrosis, also known as osteonecrosis, is a disease caused by the death of bone tissue due to lack of blood supply. Over a period of time, as the disease progresses, this leads to tiny breaks in the bone and the bones eventually collapse. Alcoholism, excessive use of steroids and post trauma (fracture or dislocation) are the most common causes of this disease and affect individuals of 30 to 70 years old. Also, it affects the male population more than the female population [31].

Osteoarthritis is the primary indication for $94 \%$ of total hip arthroplasties [32]. Approximately $30 \%$ of adults greater than age 65 in the USA have radiographic evidence of hip OA ( Kellgren \& Lawrence grade 2, 3 or 4), while the prevalence of symptomatic, radiographically documented hip OA is almost 45 in this age group [33] [34]. AfricanAmericans appear to have a similar or even slightly higher prevalence of hip OA than 
whites [34]. Age, genetic predisposition in certain families, congenital or developmental hip disorders, obesity, occupational exposure to heavy weight bearing activities (e.g., farming) and jobs with bending and squatting are established risk factors for the development of hip OA [35-39]. The direct medical cost attributable to OA in the USA exceeds $\$ 15$ billion per year [40] [41]. In addition, persons with OA are two to four times more likely than those without OA to retire early, to report reduction in work hours or to be unable to find work due to illness [42].

\subsection{4: Non-operative management of $\mathrm{OA}$ and need for THR}

The management of hip OA includes non-pharmacologic approaches, such as patient education and self-help programs, social support, weight loss (if patient is obese), assistive devices and exercises aimed at stretching, quadriceps strengthening and aerobic fitness. Multiple pharmacologic therapies can be used including acetaminophen, topical analgesics, non-steroidal anti-inflammatory drugs (NSAIDs), COX-2 inhibitors, opioid analgesics and injections of corticosteroids or hyaluronic acid.

These measures may reduce pain and improve functional status but do not slow the progression of cartilage loss and joint damage. Hence, many patients with hip OA ultimately must decide whether to elect surgical management. The safety of NSAIDs and COX-2 inhibitors are under intense scrutiny, which may increase demand for surgery. The primary surgical option for advanced hip OA is THR. In one study, $36 \%$ of patients with established, symptomatic hip OA elected THR over five years of follow up [43].

\subsection{Introduction to Hip implants}

The anatomy of the hip joint and the forces acting on it dictate the modeling and design of hip implants. There are two main parts to a THR implant. The femoral 
component fits into the top of the femur and replaces the ball of the ball-and-socket joint. The acetabular cup sits in the pelvis and replaces the socket. The diaphragm and micrographs of THR are shown in Figure 8.
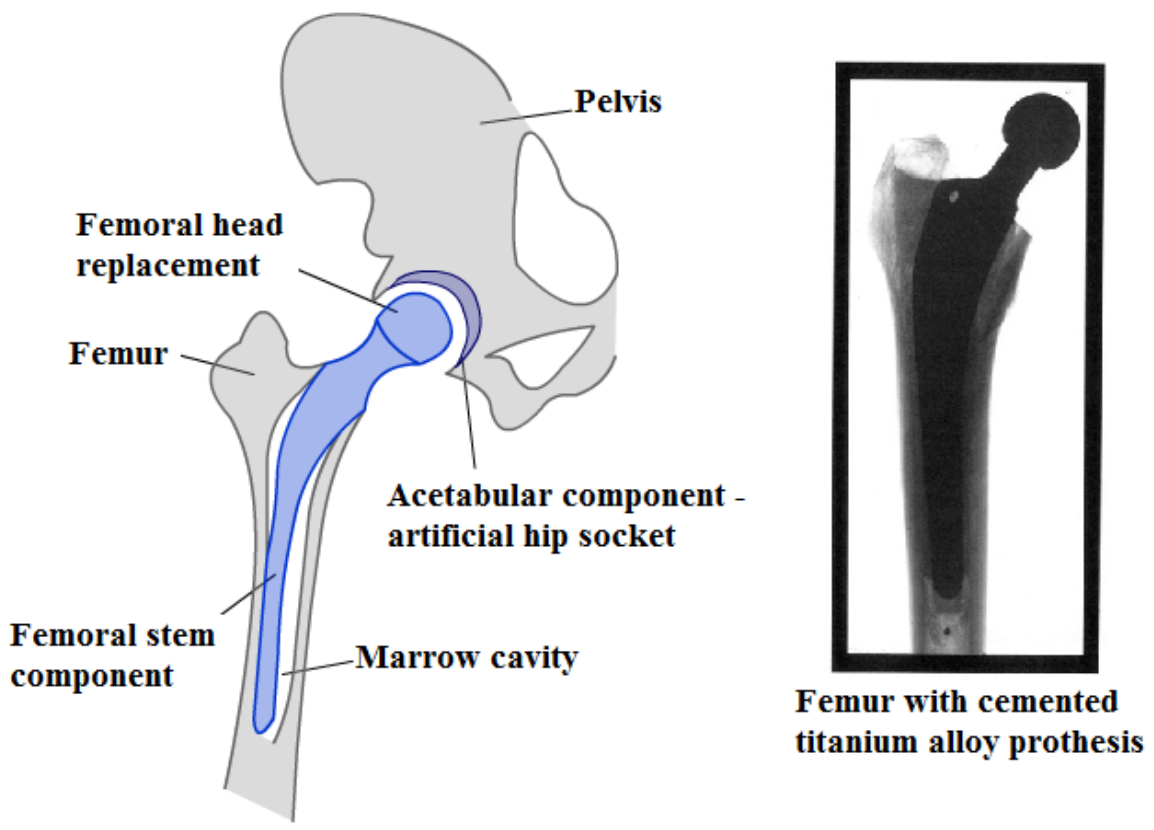

Figure 8 Diaphragm and radiograph of a hip replacement [44]

The femoral component is fitted by inserting it into the shaft of the femur, which is prepared by hollowing out a section to fit the stem of the implant. The femoral stem essentially replaces bone in the femur and has a structural role that requires it to match, as closely as possible, the strength and toughness of the natural bone. Also, the shape and the coefficient of friction of the surface of the femoral head components are significant to have an exact fit with the replacement socket. The acetabular cup sits in the hip socket and replaces the worn cartilage. Therefore, this part must itself have a low wear rate and also minimize wear of the femoral head. 
The requirements for the femoral head component are that it must have a very particular shape, which fits exactly within the replacement socket, and the surface should have a low coefficient of friction. The acetabular cup component sits in the hip socket and replaces the worn cartilage. Therefore, this part must itself have a low wear rate and must also minimize wear of the femoral head component.

Several materials have been considered for implants, including ceramics, polymers and metals. Each material has its own pros and cons. For e.g., although 70\% of the bone weight can be replaced by ceramic, HA ceramics are not suitable for femoral stem replacements, as they are very brittle. Similarly, polymers are prone to suffer from creep and early fatigue failure. Metallic alloys are the most commonly used materials in the hip implants, but their stiffness is higher than that of the bone, which results in softening of bone and this phenomenon is called stress shielding. The causes and effects of stress shielding are explained in detail in the next section.

\subsection{Stress Shielding}

If the implant is much stiffer than the bone, then the implant will bear more of the load. Because the bone is shielded from much of the stress being applied to the femur, the body will respond to this by increasing osteoclast activity, causing bone resorption. Metals are generally used because they typically have a high Young's Modulus, are tough and ductile, meaning they yield before breaking, and have good fatigue resistance. They do, however, tend to be much stiffer than the bone, which lead to stress shielding [45].

\subsection{History of total hip replacement}

In 1938, Philip Wiles (London) developed the first total hip implant and performed THR, which consisted of a stainless steel cup and head. The cup was fixated using screws 
and the head was fixated using a stem; which was further fixed to the neck of the femur bone by a bolt. Clinical results of this development were not known due to the intervention of World War II. Years later, metal-on-metal combinations were introduced by Mckee, Farrar and Ring (UK) and Haboush, Urist and Mcbride (USA).The results from such implants were not satisfactory due to loosening and high wear of the components as a result of increased frictional torque [46]. These MOM prostheses were manufactured to give a matching femoral head and acetabular cup with no clearance (small space between the head and the cup). It was shown that introducing a small clearance between the two components could reduce friction by creating a polar bearing [46].

It was not until 1958 when Sir John Charnley implanted the first metal-on-polymer THR. The first polymer Charnley chose was PTFE articulating against a stainless steel head, which only survived two years due to the rapid wear of PTFE. In 1961, he adopted ultra-high molecular weight polyethylene (UHMWPE), which provided a low interfacial friction against a metal head. This prosthesis was called "Low Friction Arthroplasty." Clinical results have encouraged the use of Charnley prosthesis. His concept is still being used to date in less active elderly patients [47].

Metal-on-metal bearings gained potential in the late 1980s after the long-term survivorship and low wear of such prosthesis. It was thought that the improved tolerances and better finishes can be achieved with better manufacturing technologies. This would improve the outcome of metal-on-metal prosthesis.

In the early 1970s, Boutin from France, developed first ceramic-on-ceramic (COC) total hip replacements [48]. Ceramics are highly inert materials and are widely used in 
Europe due to the good surface finish and excellent resistance to wear in vivo [49]. Although COC has shown enhanced wear performance, there are still concerns about the incidental fracture of the ceramic material [50]. The further developments of COC prostheses will be discussed in subsequent sections.

A newly developed bearing in the THR is the ceramic-on-metal (COM) combination. COM consists of a ceramic head that articulates against Cobalt Chrome (CoCrMo) lines. This bearing offers enhanced wear performance, which has been proven successful in an in-vitro hip simulator study [51]. The smoothness of the ceramic head helps reduce abrasive wear on the metallic liner. It provided reduced risk of liner fracture, which was noticed in COC bearings and also reduced levels of ion concentration compared to MOM bearings [52].

\subsection{Fixation techniques of hip implant}

The process of fixing the hip implant in the human body, involves the femoral stem component be inserted into the femur bone, which is prepared by drilling, to fit the component. This is traditionally bound to the bone with polymer bone cement called poly-methyl methacrylate (PMMA). In addition to fixing the implant in place, the cement helps with even distribution of load between the implant and bone. The drawback with this method is however, during the curing process (hardening through cross-linking of polymer chains). During curing, a large amount of heat is released that can cause necrosis (cell death) in the bone around the implant and may also lengthen recovery time [45].

An alternative fixation method is to introduce a porous surface layer to the implant, which encourages bonding by allowing the bone to grow into the pores. The bone and implant therefore become integrated, meaning that the implant is less prone to loosening. 
A further modification is to coat the implant with a layer of HA. Since its chemical composition is similar to that of bone mineral, the coating enhances bone growth. Many surgeons now favor these un-cemented implants, as they give a quicker recovery time, with many patients able to put weight on their hip the day after surgery.

Coating with HA does raise some concerns. The coating is done at an elevated temperature. As the implant cools, due to different rate of thermal expansion, it generates thermal stress and causes cracking of the surface on the implant. In an attempt to match the thermal expansion coefficients (and to avoid cracking), manganese was also considered as an additive.

\subsection{Material history of hip implants}

The components of hip implants are made from different materials, and choosing materials with appropriate properties is the key part of their success. The choice of materials depends on several factors, which include age of the patient, level of physical activity and the surgeon's preference. In the past four decades, surgeons were using metal and plastic, MOM, COC and metal with highly cross linked polyethylene. The most common materials used are titanium, stainless steel and cobalt chrome alloys. These three types of alloys were considered by surgeons for several decades. Each material has its own advantages and limitations. The highly cross linked polyethylene implants are newly developed and so limited information is available on their performance in terms of their wear rate.

MOM implants gained popularity due to their very low wear rate and are considered to last longer. Concerns were raised, as metal ions are released into blood, leading to increased risk of toxicity, cancer and similar diseases. Later, COC implants were 
considered due to lower wear rate than MOM implants and are also scratch resistant and smoother. However, there were concerns for this type of implant as well, as ceramics tend to break or crack easily under shock load.

\subsubsection{Metal Implants}

Metals are the most commonly used hip implant materials due to high Young's Modulus, toughness, ductility and high resistance to fatigue. Among the metallic implants, Ti6Al4V and CoCr alloys are most widely used for hip and knee implants. Rather than using commercial purity titanium alloy, the alloy Ti6Al4V (Titanium with $6 \%$ Aluminum and $4 \%$ Vanadium by weight) is often used, as it gives increased toughness and improves fatigue resistance. Stainless steel has also been used in some cases. Co-Cr-Mo alloy was introduced as an alternative, since it has better wear properties than stainless steel. However, it is harder, meaning it is much more difficult to machine.

A major downside of metal hip implants is that they tend to be much stiffer than bone. Clinical studies and literature from the past two decades suggested that there is a mismatch of stiffness between the implant and femur bone, increasing osteoclast activity, causing bone resorption [3-5]. This contributes to implant failure due to bone softening, referred to as the stress shielding effect.

\subsubsection{Composite Implants}

Another alternative to metallic and ceramic implants is composite material based hip implants. Previous studies using unidirectional composite materials have shown the potential to eliminate the stress shielding effect, but they lacked the modulus and strength in critical locations to solve this issue [6]. In addition, transverse properties are low and 
de-lamination and micro cracking initiate at relatively low loading values. Prostheses made from polymer composites have the potential to meet spatially varying mechanical requirements, such as strength and stiffness [53]. However, while a more compliant hip implant reduces stress shielding, it must maintain a minimum level of stiffness not to cause residual pain due to low-amplitude oscillatory micro-motion [54].

Although there are several options available for THR implants, each one has its own merits and demerits. To overcome the major issues of stress shielding and metal ion leaching in currently used hip implants, 3D Woven Composite materials are being investigated as an effective alternative in the present work. The detailed description, design and analysis of 3DWCs is explained in the following chapters. 


\section{CHAPTER 3- 3D Woven Composites}

\subsection{Introduction on composite material design}

Several theoretical formulations have been developed for composite material design. To determine the various properties of composite materials theoretical models like Rule of Mixtures (ROM), Inverse ROM, and ROM with efficiency factor, Hart Smith’s 10\% rule and classical laminate analysis exist. ROM can be used to predict the Young's Modulus, density, Poisson's ratio and strength (UTS) of the material.

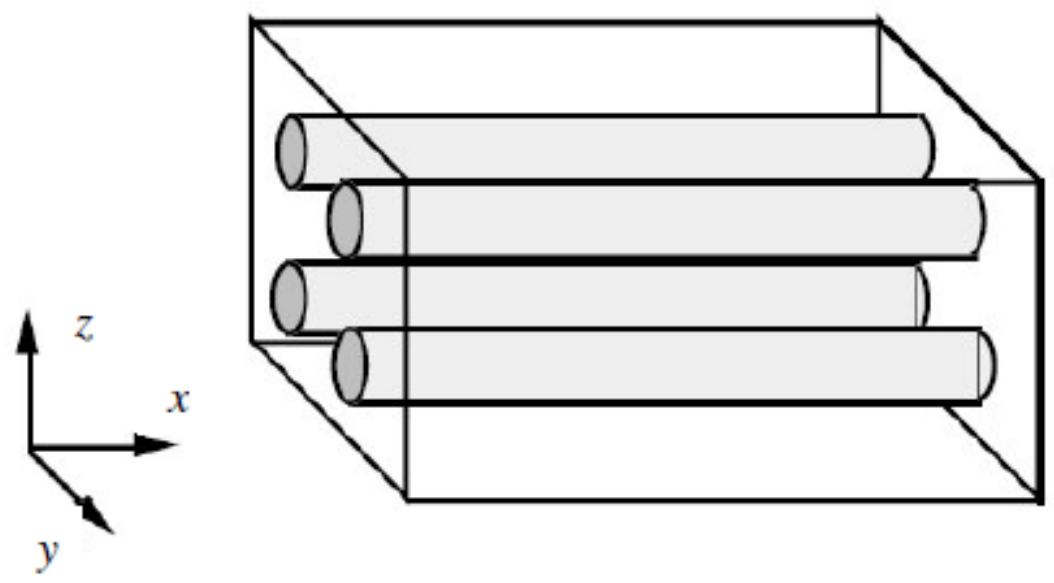

Figure 9 Ideal Unidirectional RVE

Most theoretical formulations are based on the fiber volume fraction of the composite. Composite manufacturers usually provide the fiber weight fraction. Hence, the fiber weight fraction is converted to the required fiber volume fraction using the following formula: 


$$
V_{f}=\frac{\frac{w_{f}}{P_{f}}}{\left(\frac{w_{f}}{P_{f}}+\frac{w_{m}}{P_{m}}\right)}
$$

ROM assumes that fibers are unidirectional and the Young's Modulus is predicted in fiber direction using the following formula:

$$
E_{c=} E_{f} V_{f}+E_{m} V_{m}
$$

ROM with efficiency factor or Krenchel factor is used to predict the effect of fiber orientation on stiffness. The reinforcing efficiency factor (composite efficiency factor) is given by the formula:

$$
\eta_{\theta}=\sum a_{n} \operatorname{Cos}^{4} \theta
$$

where $a_{n}$ is proportional to the total fiber content and $\theta$ is the angle of fibers.

Hence, $\quad E_{c=} \eta_{\theta} E_{f} V_{f}+E_{m} V_{m}$

Eq 4

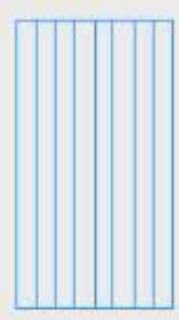

$\eta=1$

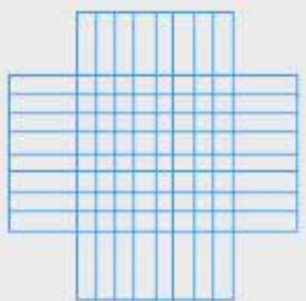

$\eta=0.5$

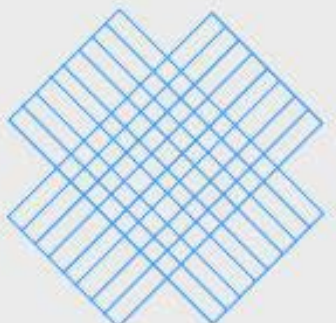

$\eta=0.25$

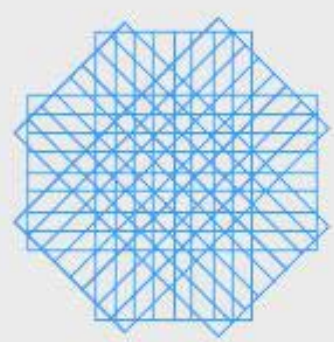

$\eta=0.375$

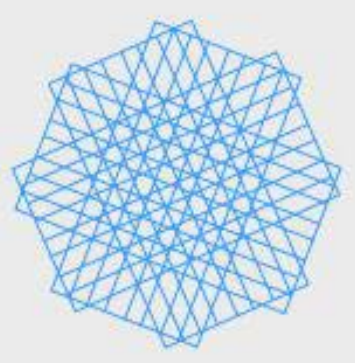

$\eta=0.375$

Figure 10 Efficiency Krenchel Factor Diagram 
In 1993, Heart Smith developed an empirical formula to consider ply orientation. Each of the $45^{\circ}$ for $90^{\circ}$ ply is considered to contribute to one tenth of the strength or stiffness of a $0^{\circ}$ ply to the overall performance of the laminate.

$$
\begin{gathered}
E_{x}=E_{11}\left(0.1+0.9 *\left(\% \text { of plies at } 0^{\circ}\right)\right. \\
\sigma_{x}=\sigma_{11}\left(0.1+0.9 *\left(\% \text { of plies at } 0^{\circ}\right)\right. \\
G_{x y}=E_{11}\left(0.028+0.234 *\left(\% \text { of plies at } \pm 45^{\circ}\right)\right.
\end{gathered}
$$

These formulations are rapid and provide a reasonably accurate estimate for classical composites or composites with uniform fibers.

Using classical laminate analysis, classical properties $E_{11}$ (longitudinal stiffness), $E_{22}$ (transverse stiffness), $\vartheta_{12}$ (major Poisson's ratio) and $G_{12}$ (in-plane shear modulus) of a ply can be estimated. Longitudinal stiffness using ROM is given by:

$$
E_{c=} E_{f} V_{f}+E_{m}\left(1-V_{f}\right) \quad \text { Eq } 6
$$

Transverse stiffness is determined using the Inverse ROM (Reuss Model) as:

$$
\begin{array}{ll}
\frac{1}{E_{c}}=\frac{V_{f}}{E_{f}}+\frac{V_{m}}{E_{m}} & \text { Eq } 7
\end{array}
$$

Major Poisson's ratio using ROM is determined as:

$$
\vartheta_{12}=\vartheta_{f} V_{f}+\vartheta_{m}\left(1-V_{f}\right) \quad \text { Eq } 8
$$

In-plane shear modulus is determined using Inverse ROM as:

$$
\frac{1}{G_{12}}=\frac{V_{f}}{G_{f}}+\frac{\left(1-V_{f}\right)}{G_{m}}
$$

The above mentioned empirical formulations are an approximation and are very far from estimating the actual properties of woven composites and in particular, 3DWCs. Woven composites are designed using their properties, as long as experimental data is available for all types of fiber/matrix combinations used in the laminate [55]. 


\subsection{Transverse Stiffnes -Elastic Moduli $E_{y}$ and $E_{z}$}

The analytical prediction of the transverse stiffness in unidirectional composite appears more difficult than the axial case. Although the system can still be represented by the "slab model", this simplified model shows a serious drawback. The expression derived from the conventional approach of the slab model that in the case of transverse loading is also called the "equal stress model”.

The relation derived from the slab model approach is:

$$
\frac{1}{E_{y}}=\left(\frac{1}{E_{f}}\right) V^{f}+\left(\frac{1}{E_{m}}\right)\left(1-V^{f}\right)
$$

This is the ROM relation for Ey usually called Inverse ROM. Comparing equation (6) with the expression for $E_{y}$ in equation (10), it is seen that it relates the reciprocal of the extensional moduli with the volume fraction rather than the moduli themselves. This expression is similar to the expression for the equivalent stiffness of a pair of springs in series.

The non-uniform distribution of stress and strain during transverse loading implies that the simple equal stress model appears to be inadequate to evaluate the transverse elastic properties and more in detail the slab model gives an underestimate value of the Young's modulus.

The most successful empirical expression to obtain more accurate estimates of $\mathrm{E}_{\mathrm{y}}$ (and $E_{z}$ ) has been proposed by Halpin-Tsai, in 1967. Although it is not based on a rigorous theory of elasticity it takes into account the enhanced capability of fiber load bearing, relative to the equal stress model.

The relation for the transverse stiffness is: 
$\frac{E_{y}}{E_{m}}=\frac{1+\psi_{\eta f}}{1+n f}$

Eq 11

where

$$
\eta=\frac{\gamma-1}{\gamma+\psi}
$$

and

$$
\gamma=\frac{E_{f}}{E_{m}}
$$

where $\psi$ is a measure of fiber reinforcement of the composite material and is dependent on fiber geometry, packing geometry, and loading conditions. Obtaining accurate values for $\psi$ is the most difficult part of using the Halpin-Tsai equations. The Equation (6) gives the correct values in the limits of $\mathrm{f}=0$ and $\mathrm{f}=1$ ( $\mathrm{f}$ is the fiber content) and in general shows a good agreement with experimental data over the complete range of fiber volume fraction. The value of $\mathrm{y}$ is then an adjustable parameter and its magnitude is generally of the order of unity. Approximations for $\psi$ are:

a) Circular fibers in square array (excellent results for $V_{f} \sim 0.55$ )

$\psi=2$ for $\mathrm{E}_{\mathrm{y}}$

$\psi=1$ for $\mathrm{G}_{\mathrm{xy}}$

b) Square fibers in square array (good results for $\mathrm{V}_{\mathrm{f}}<=0.9$ )

$\psi=2$ for $\mathrm{E}_{\mathrm{y}}$

$\psi=1$ for $\mathrm{G}_{\mathrm{xy}}$

c) - Rectangular cross section in diamond array (good results for $V_{f}<=0.9$ )

$\psi=2 \mathrm{a} / \mathrm{b}$ for $\mathrm{E}_{\mathrm{y}}$ 
$\psi=1.73 \log (\mathrm{a} / \mathrm{b})$ for $\mathrm{G}_{\mathrm{xy}}$

where $\mathrm{a}=$ width of fiber, and $\mathrm{b}=$ thickness of fiber.

\subsection{Additional approaches in the design of composite materials}

\subsubsection{Experimental methods}

Experimental testing is another method used to determine bulk material properties of woven composites. Several researchers conducted experimental studies using 3-point bending test, quasi static tensile tests, a short beam shear test and even non-destructive tests like resonance frequency technique to determine load carrying and energy absorption capabilities, young's modulus and other mechanical properties of composite materials [9] [56-62]. Experimental testing is the best method to predict bulk material properties of composites accurately and thus eliminating the need for micromechanics modeling. Unfortunately, generating experimental data for several configurations requires a large investment and is time consuming. Also, it is highly impossible to build and test several architectures with all the variations.

\subsubsection{Finite Element Analysis (FEA)}

FEA is one of the most widely used and accepted methods in the analysis of engineering systems. Several researchers in the past and present have been performing microanalysis of 3DWCs to determine the bulk material properties. The manufacture of fibers involves methods such as dry-fiber processing techniques like the vacuum bag or platen method. Due to the complexity involved in modelling the realistic shapes of fibers and matrix, many researchers in early 90's, made an approximation to idealize these cases by representing fibers with regular circular cross sections and matrix as regular rectangular or square cross sections. These idealistic models predicted the bulk material 
properties, which agree closely with analytical formulations ROM, 10\% Smith’s rule. But these estimated properties deviated more than $+/-10 \%$ when compared to experimental results. Above all, these approximations failed to capture the property changes due to variations in the architectures.

Another group of researchers concentrated on the effect of deformed and kinks in fibers during compaction process to the final bulk material properties of the woven composites [9] [10] [57] [63]. These researchers developed numerical models and their results are in close agreement with experimental values within $+/-3 \%$. They concluded that it is not wise to idealize the cross-sections ignoring the compacting process for finite elemental analysis.

Wang et al. and other researchers developed physics based models to determine the deformed/smeared shape of compacted fibers. These models are compared with SEM and micro CT images of experimental samples and are in agreement with physics based models. Furthermore researchers, Wang et al. and Zhou et al. developed single and multidigital element method as opposed to traditional finite elemental analysis [57] [64] [65]. The digital element approach reduced burden on the need for high computations. This group worked for more than a decade and half in developing digital element method, improve, perform feasibility studies and validated the process experimentally. This final compacted physics based models represented the realistic geometries of the fiber and matrix. This group along with Endel et al. 1996, extended further to determine the bulk material properties using b-Spline approximation method instead of pure Finite Element Analysis. This method reduced computational time and resources needed in solving the stiffness matrices within FEA drastically [66]. Hence, the method of multi-digital 
elements and b-spline approximation methods are adopted in this study to determine the bulk material properties of 3DWCs.

\subsubsection{B-Spline Finite Element Formulation [67]}

B-splines provide a methodology to approximate a given set of points with smooth polynomial functions. The basis functions are represented by piecewise polynomials and the approximation provides a high degree of continuity depending on the basis used. For example, continuity is obtained when quadratic basis is used and continuity is obtained when cubic basis is used and so on. It is to be noted that the Lagrange interpolations, which are used in traditional FEM are continuous irrespective of the basis used. B-spline basis functions are classified into uniform, non-uniform and NURBS. Uniform b-spline basis functions have equally spaced nodes in the parameter space and the basis functions are periodic. Adjacent nodes in non-uniform b-splines are not equidistant in the parameter space. NURBS are generalization of non-uniform b-splines, where the basis functions are rational functions and are capable of representing several analytical curves exactly. In this work, uniform b-splines are used for approximating the solution space. The discussion on b-splines in the remaining of this chapter is limited to uniform bsplines.

The b-spline basis functions have compact support and lead to banded stiffness matrices. b-spline approximation provides high order of continuity and is capable of providing accurate solutions with continuous gradients throughout the domain. This is a major advantage over standard finite element shape functions. The b-spline basis functions form a partition of unity and are able to accurately represent the state of constant field in the domain of interest. This property is very important for convergence 
of the approximate solutions provided by b-splines. The basis functions used are polynomials and accurate integration can be performed by using Gauss quadrature. The b-spline approximation has good reproducing properties and when a 57 cubic b-splines are used for approximating the solution, all the polynomial solutions up to third order can be represented exactly. B-spline shape (basis) functions are traditionally constructed using recursive definition [68]. The parameter space is partitioned into elements using a knot vector (equivalent to a collection of nodes). Very general methods are available to insert knots and elevate the order (or degree) of the polynomial basis functions. This framework therefore can be used to implement h-p- and k-refinement and has been studied in the context of iso-geometric analysis by Hughes et al [69]. Instead of using recursive definitions as in geometric modeling applications, polynomial definitions for bspline basis functions are used here. Furthermore, it is convenient to define an independent parameter space for each element in the grid instead of a continuous parameter space (or knot vector) for all elements. Therefore it is assumed here that for each element in the grid, parameter values vary within as is done for traditional isoperimetric finite elements. This is illustrated for the one-dimensional case in Figure 11 where a cubic b-spline approximation is shown. In Figure 11, element is defined between vertex nodes 2 and 3, but the approximation defined over (referred to as its span) is controlled by four nodes (1-4), which can be refer to as its support nodes. 


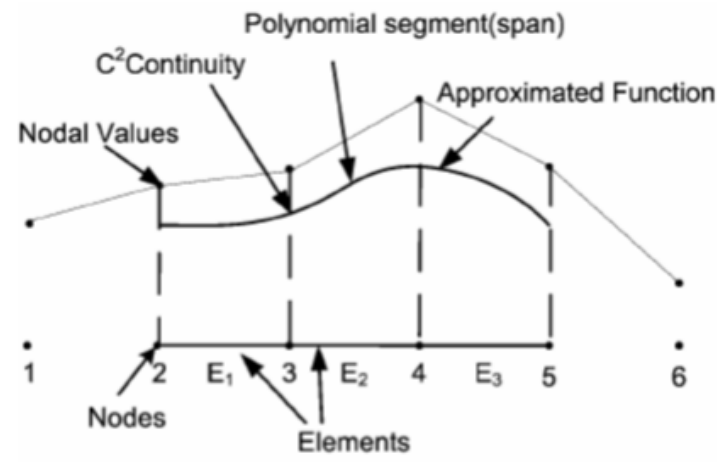

Figure 11 Approximation using 1D cubic b-splines

\subsubsection{One Dimensional B-Spline Elements:}

The polynomial expressions for the basis functions can be derived using the continuity requirements between adjacent elements. $\mathrm{A} \mathrm{k}^{\text {th }}$ order $\mathrm{b}$-spline has $\mathrm{k}+1$ support nodes with some of the support nodes lying outside the element. The span corresponding to the element is defined by the basis functions of all the support nodes. The basis functions are polynomials of the form $N_{i}=\sum_{j=0}^{k} a_{i j} r^{j}$

whose coefficients are determined using continuity requirements. Since the parameter range for the element is $r \in[1-, 1]$, the parameter value for first support node is $r=-$ $(\mathrm{k}+1) / 2$ and for the $(\mathrm{k}+1)^{\mathrm{th}}$ support node is $\mathrm{r}=(\mathrm{k}+1) / 2$ for the parameterization to be uniform. The approximated function is a linear combination of the basis functions within each element so that the span of the element $\mathrm{e}$ is represented as

$$
f_{e}(r)=\sum_{i=0}^{k} N_{i} u_{i}=\sum_{i=0}^{k}\left(\sum_{j=0}^{k} a_{i j} r^{j}\right) u_{i}
$$

In the equation (12) $u_{i}$ are the support nodal values and it is assumed for convenience that the first support node for element has index 0 . b-spline approximation of order $\mathrm{k}$ has 
$\mathrm{b}^{(\mathrm{k}-1)}$ continuity at the junction between adjacent elements. This continuity requirement can be stated as

$$
\frac{\partial^{m} f_{e}(1)}{\partial r^{m}}=\frac{\partial^{m} f_{e+1}(-1)}{\partial r^{m}}, m=0,1 \ldots . k-1
$$

where, e and $\mathrm{e}+1$ are adjacent elements whose derivatives are evaluated at the end point $(\mathrm{r}=-1)$ and start point $(\mathrm{r}=1)$ respectively. $\mathrm{m}=0$ represents the continuity equation. Using Eq. (13), the continuity equations can be expressed as

$$
\begin{array}{cc}
\left(\sum_{j=0}^{k} \frac{j !}{(j-m) !} a_{0 j}\right) u_{0}+\sum_{i=1}^{k}\left(\sum_{j=0}^{k} \frac{j !}{(j-m) !} a_{i j}-\sum_{j=0}^{k} \frac{j !}{(j-m) !} a_{i j}(-1)^{j-m}\right) u_{i}- \\
\left(\sum_{j=0}^{k} \frac{j !}{(j-m) !} a_{k+1 j}(-1)^{j-m}\right) u_{k+1}=0 & \text { Eq } 14
\end{array}
$$

Since this equation (14) is valid for arbitrary values of Ui, I varying from $0 . . \mathrm{k}$, the coefficients corresponding to each should vanish. This results in a set of linearly independent equations. Since equations are obtained for each value of , a total of linearly independent equations are obtained. An additional equation stating that $\mathrm{Ni}$ used to obtain $\mathrm{k}+1$ basis functions form a partition of unity needed to solve for all coefficients of the basis functions. Using this approach, the basis functions for quadratic and cubic b-splines can be derived as shown in Eq. (15) and the basis functions plotted.

\subsubsection{Quadratic B-spline element}

The shape functions for quadratic b-spline element are constructed using the procedure explained above. Quadratic b-spline element in one-dimension has three nodes and hence three shape functions. The corresponding equations are shown below:

$$
N_{1}=\frac{1}{8}\left(1-2 r+r^{2}\right) ; N_{2}=\frac{1}{8}\left(6-2 r^{2}\right) ; N_{3}=\frac{1}{8}\left(1+2 r+r^{2}\right) \quad \text { Eq } 15
$$

\subsubsection{Cubic B-spline element}

It can be seen that, unlike traditional finite element shape functions, the b-spline basis functions are not unity at the corresponding node and do not vanish at other nodes. 
Therefore, these basis functions do not satisfy the Kronecker-delta property and the approximation constructed using these basis functions does not interpolate nodal values.

$$
\begin{gathered}
N_{1}=\frac{1}{48}\left(1-3 r+3 r^{2}-r^{3}\right) ; N_{2}=\frac{1}{48}\left(23-15 r-3 r^{2}+3 r^{3}\right) \\
N_{3}=\frac{1}{48}\left(23+15 r-3 r^{2}-3 r^{3}\right) ; N_{4}=\frac{1}{48}\left(1+3 r+3 r^{2}+r^{3}\right) \quad \text { Eq } 16
\end{gathered}
$$

\subsubsection{Two and Three Dimensional B-Spline Elements}

The basis functions for the higher dimensional b-spline elements are constructed by taking product of the basis functions for one-dimensional b-splines. In a typical grid used corresponding to cubic b-spline elements where the span corresponding to each element is controlled by 16 support nodes. The grid elements used here are regular quadrilaterals (rectangle/square) or regular hexahedra (cube/cuboid), hence the 60 mapping for geometry of elements from parameter space to the physical space is linear. Note that even if an iso-parametric formulation is used the mapping will degenerate to a linear map because the element geometry in physical space is regular and can be obtained by scaling the element geometry in parametric space.

Hence, in this chapter a brief review of the methods of design of composite materials is presented. The specific cases of the approaches used to design 3DWCs is presented in the next chapter. 


\section{CHAPTER 4-Design of 3DWCs using VTMS}

In this chapter, the FEA based software, VTMS has been used to design the 3DWCs. Initially, FEA of a unit composite cell analysis is performed as a proof of concept. This is followed by extended FEA analysis of 3DWCs to validate VTMS and BSAM methods. Different architectures and materials have been analyzed. Finally, the developed architectures and materials are used in the macro-analysis of a hip implant.

\subsection{Validation of FEA models}

In the validation of FEA model, a basic unidirectional unit composite cell analysis is performed and results are compared to those obtained in theory.

\subsubsection{Finite Element based Hexagonal Unit Composite Cell analysis}

A unit hexagonal composite cell is modeled in Abaqus 6.12/6.13 as shown in Figure 12. The center highlighted cylindrical part represent fiber and the rest represents matrix. For proof of concept S2- Glass is considered as fiber and poly methyl methacrylate (PMMA) is considered as matrix. Three different configurations are considered representing different fiber-matrix percent volume (PV) - 50, 60 and 70\%.

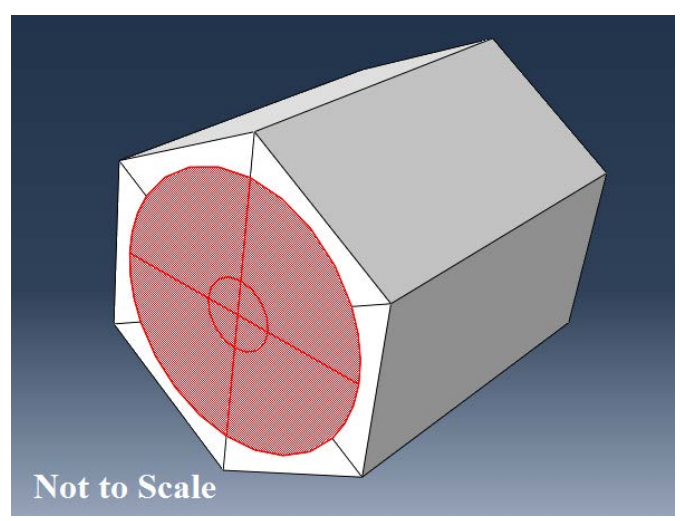

Figure 12 Hexagonal Unit Composite Cell 
Table 2 Material properties for the unit cell

\begin{tabular}{cccc}
\hline & S-2 Glass & PMMA & Units \\
\hline $\begin{array}{c}\text { Young's } \\
\text { Modulus }\end{array}$ & 86900 & 3300 & $\mathrm{MPa}$ \\
\hline Poisson's ratio & 0.23 & 0.39 & \\
\hline Density & 0.00246 & 0.00119 & $\mathrm{~kg} / \mathrm{m}^{3}$ \\
\hline
\end{tabular}

Fixed boundary condition is applied to one face and a constant displacement boundary is applied to the opposite face. 10 different displacements are applied their corresponding stress and strain values of the composite are plotted. Figure 13 shows von-misses stress and $\mathrm{U}$ - displacement in the unit cell.
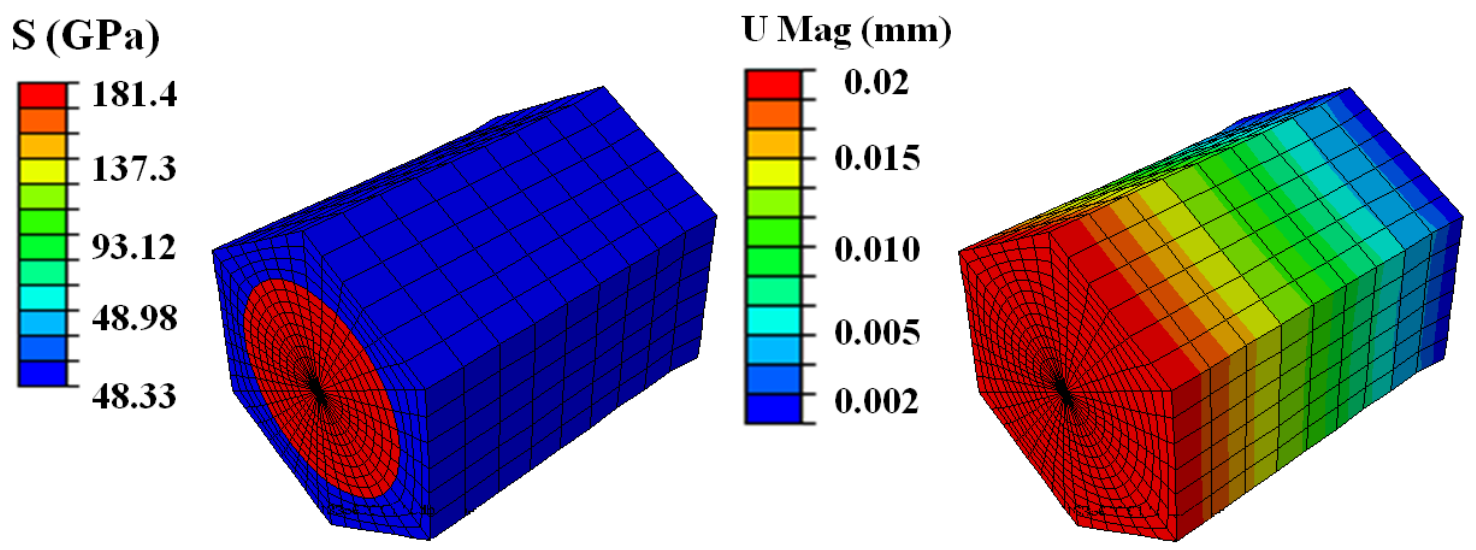

Figure 13 Von-misses stress (L) and U-displacement (R) 


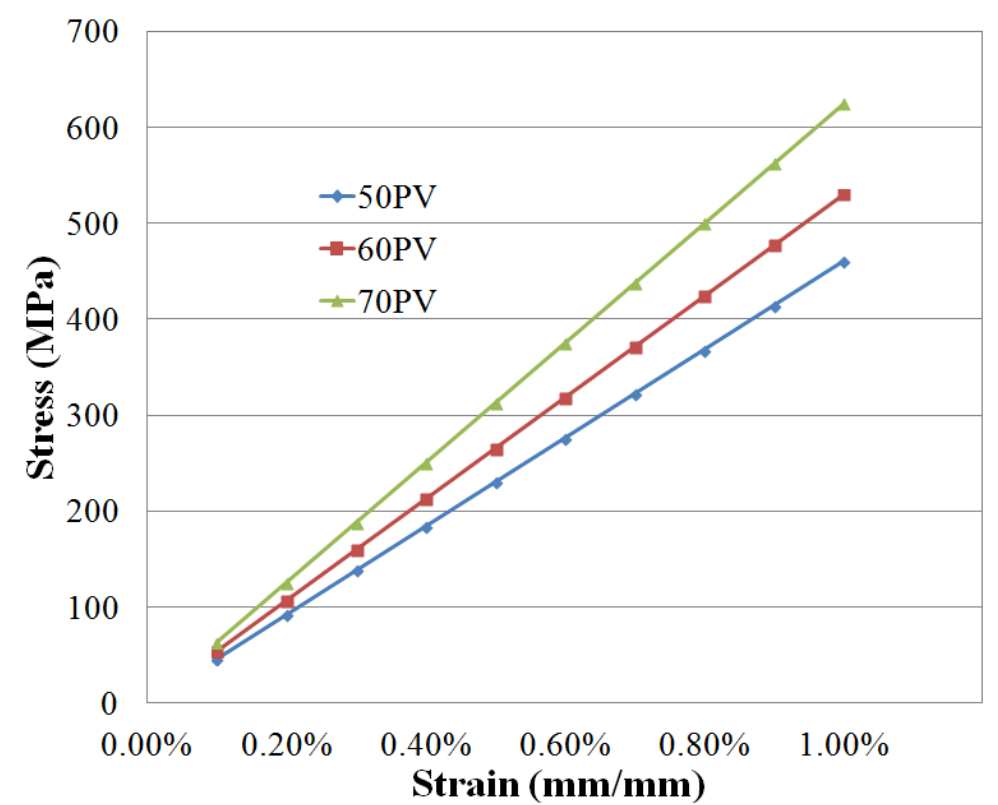

Figure 14 Stress vs Strain plots for different volume percent

From Figure 14, the values of Young's Modulus are 46000, 53000 and 62500 MPa for $50 \%, 60 \%$ and $70 \%$ fiber-matrix percent volume. These computational results matched closely with material properties of composites based on rule of mixtures.

\subsubsection{Finite Element Analysis of 3D Orthogonal Woven Composites}

In order to determine the material properties of 3DWCs, customized software Virtual Textile Morphology Suite (VTMS) and B-Spline Analysis Method (BSAM) is used. This software is developed by U.S. Air Force Research Laboratory. Predefined 3D orthogonal textile morphologies are developed in textile module within VTMS. The fiber specifications are - diameter $0.96 \mathrm{~mm}$, tow spacing $1.92 \mathrm{~mm}$, binder pattern $2 \mathrm{x} 1$ with 2 layers. These morphologies are extended, moved as needed to shift one corner of the morphology to origin. These fiber morphologies are virtually relaxed and compacted further. The method of multi-digital chains is employed to achieve this [70] [71]. This 
step generates realistic tow morphologies resulting from dry-fiber processing techniques - vacuum bag and/or platen compaction method. After relaxing and compacting the fibers to desired specifications, Tow Modify module wraps a smooth surface around them. These surfaces are then manipulated either manually or automatically to remove interpenetration errors between the two surfaces. These perform surfaces are then clipped and/or cut to an appropriate dimensions as shown in Figure 15.

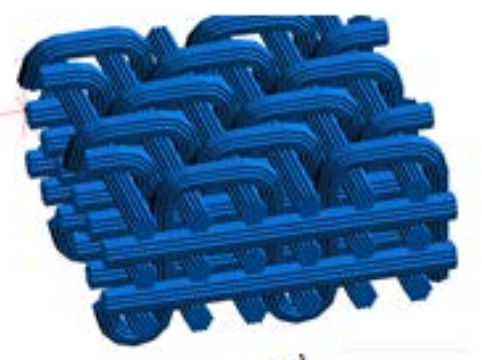

a)

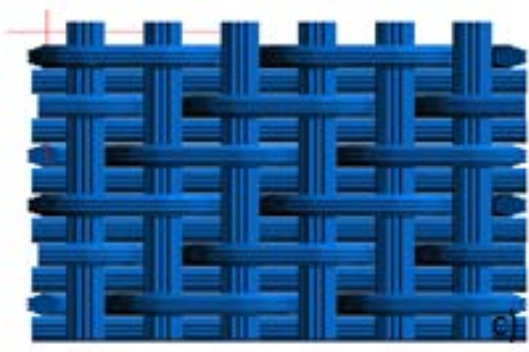

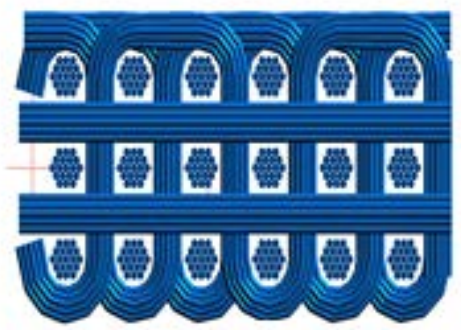

b)

c)

Figure 15 Predefined 3D Orthogonal Fibers a) Isometric View b) Front View c) Top View 


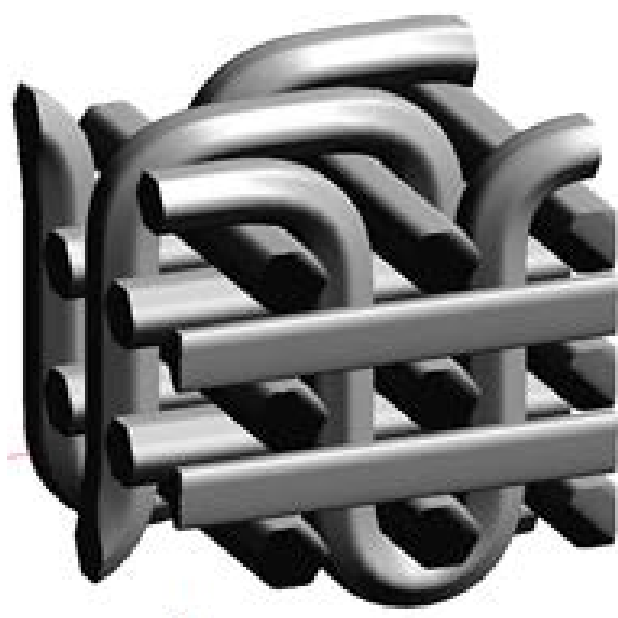

a)

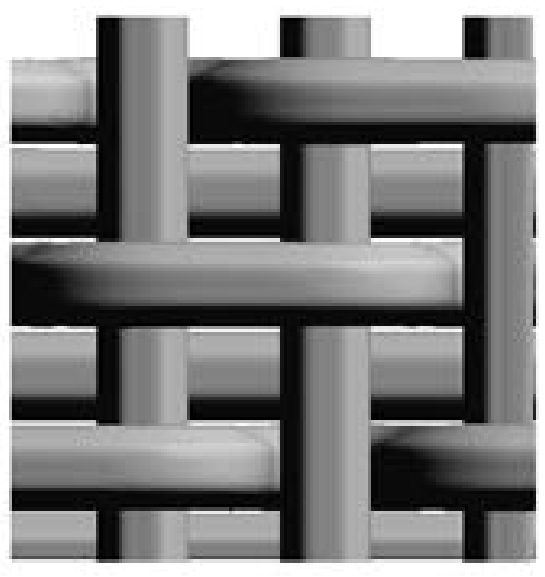

b)

Figure 16 3D Orthogonal tows after compaction a) Isometric View b) Top View

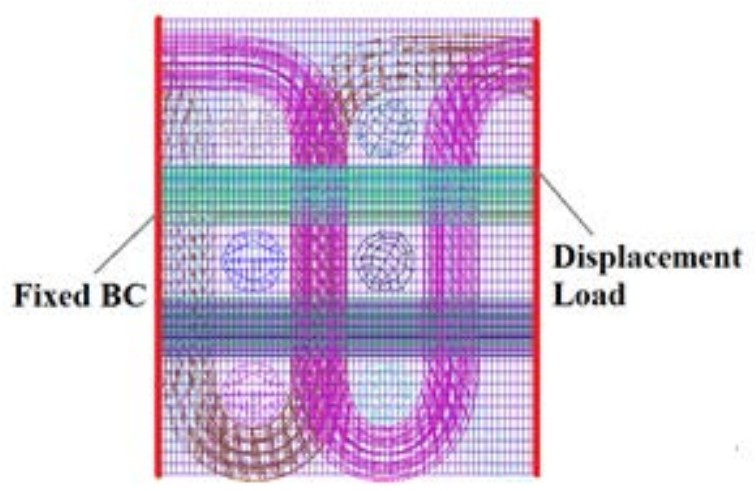

Figure 17 Matrix and fibers showing Boundary Conditions

The compacted perform surfaces are used to generate hexahedral mesh, which is compatible with BSAM. Various controls are available to adjust the quality of the mesh. A rectangular volume mesh is generated representing the resin between the tows.

These meshed parts are imported to BSAM module, assigned material properties and boundary conditions as shown in the figure. Different load conditions are applied to this composite and results are plotted. 


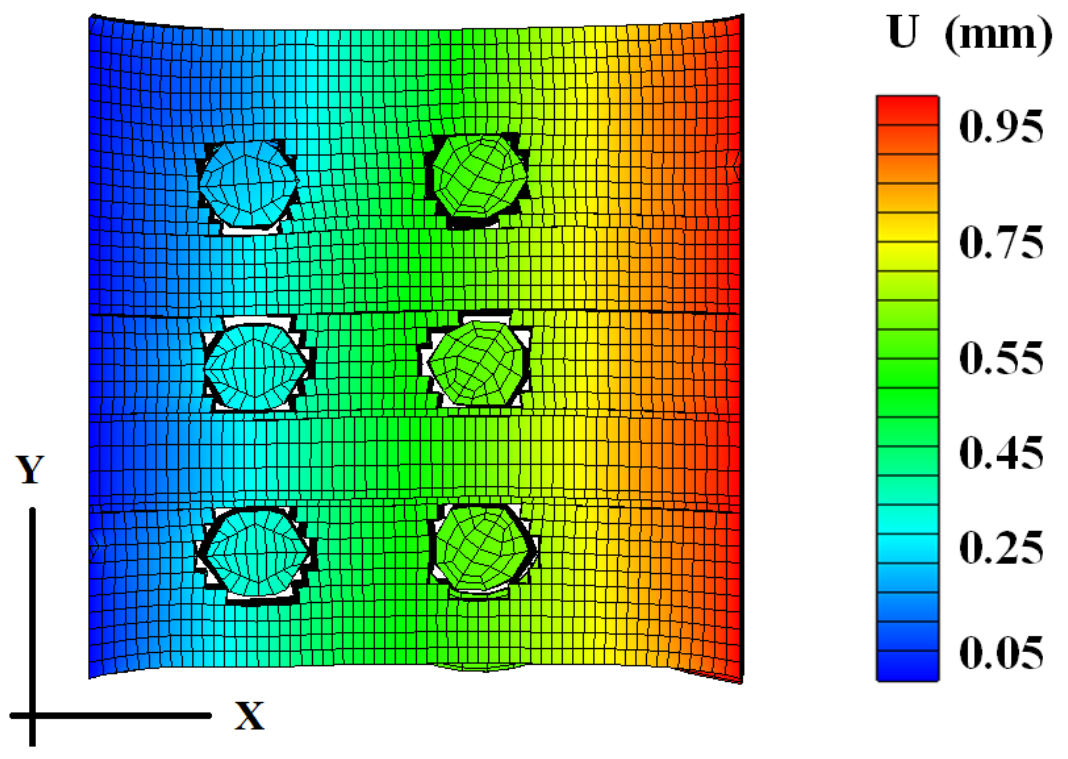

Figure 18 U-displacement of the 3DW Composite RVE

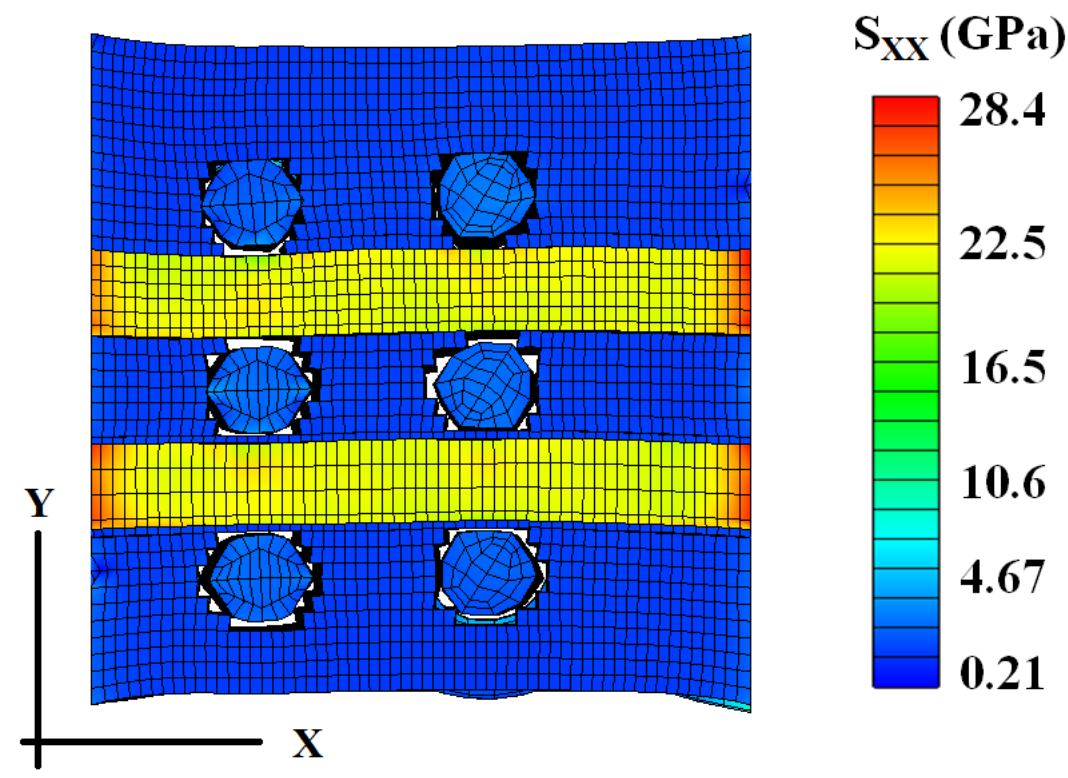

Figure $19 \mathrm{~S}_{\mathrm{xx}}$ - stress in $\mathrm{x}$ direction of 3DW Composite RVE 


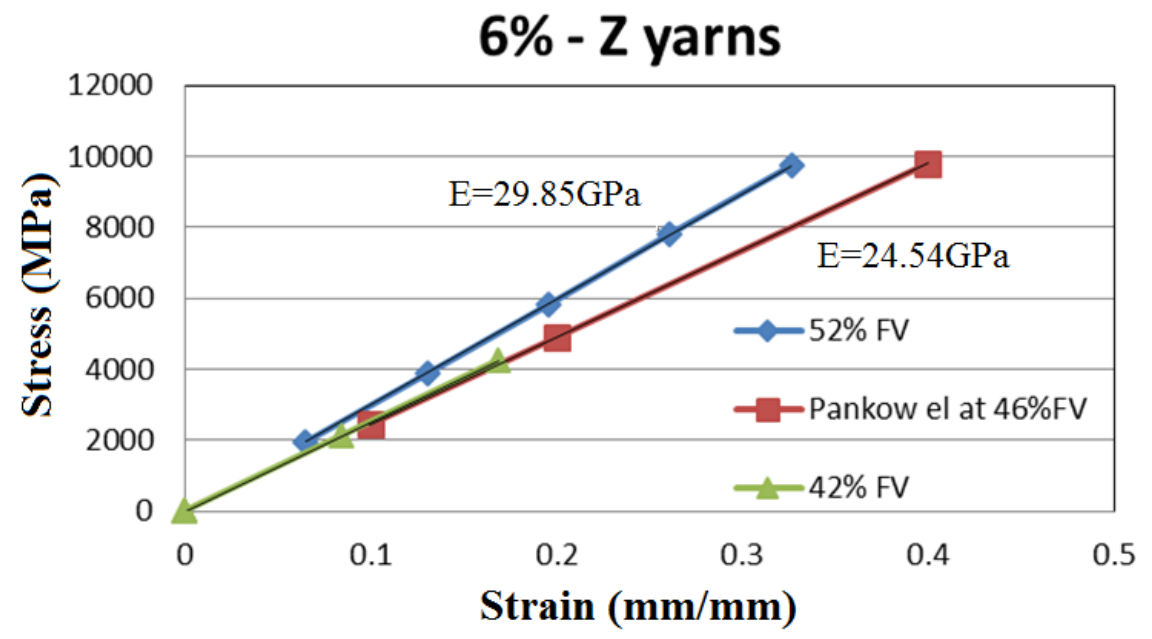

Figure 20 Stress-strain graph for $6 \% \mathrm{Z}$ yarns at $0^{\circ}$ orientation of the fibers

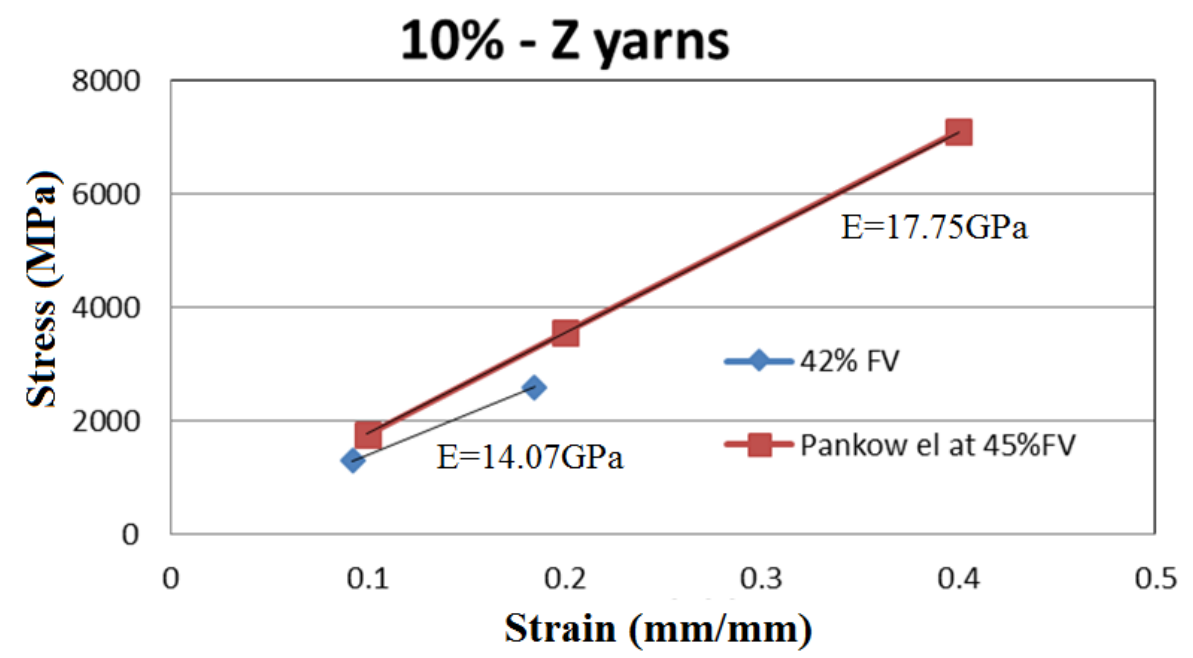

Figure 21 Stress-strain graph for $10 \% \mathrm{Z}$ yarns at $0^{\circ}$ orientation of the fibers

\subsubsection{Model Validation and Comparison of present Results with Literature}

Pankow et al. examined six different types of architectures for mechanical properties.

Of the six, the three $\mathrm{z}$ fiber designs are $3 \%, 6 \%$ and $10 \% \mathrm{Z}$ and the other three are layer to layer fiber architectures [60]. Tensile, V-notched rail shear, short beam shear and 
laterally constrained compression tests were performed on each sample until failure to capture the failure of the specimens. The material SC-15 is used as resin and S-2 glass is used for fibers. For each design a simple tensile test with three different orientations $0^{\circ}$, $45^{\circ}$, and $90^{\circ}$, are being tested. The burn out tests resulted in approximately 45 to $47 \%$ Fiber volumes.

Table 3 Material properties for VTMS analysis [60]

\begin{tabular}{lccc}
\hline Material Properties & S-2 Glass (Fiber) & SC-15 (Matrix) & Units \\
\hline Young's Modulus $\left(\boldsymbol{E}_{\mathbf{1 1}}\right)$ & 114.2 & 2.487 & $\mathrm{GPa}$ \\
Poisson's Ratio $\left(\boldsymbol{\vartheta}_{\mathbf{1 2}}\right)$ & 0.22 & 0.35 & - \\
Ultimate Stress $\left(\boldsymbol{\sigma}_{\boldsymbol{U}}\right)$ & - & 110 & $\mathrm{MPa}$ \\
Ultimate Strain $(\%)\left(\boldsymbol{\varepsilon}_{\boldsymbol{U}}\right)$ & - & 6.4 & - \\
\hline
\end{tabular}

Micro mechanical 3DW models were developed based on $6 \%$ and $10 \% \mathrm{z}$ architectures with 42 and 52\% fiber volume fractions. Same SC-15 for resin and S-2 glass fibers material properties are considered for simulations. Several displacement loads resembling the tensile loads were applied to these models along the three orientations $0^{\circ}, 45^{\circ}$ and $90^{\circ}$. The results obtained from simulations are plotted along with experimental results for comparison is shown in Figure 22. 

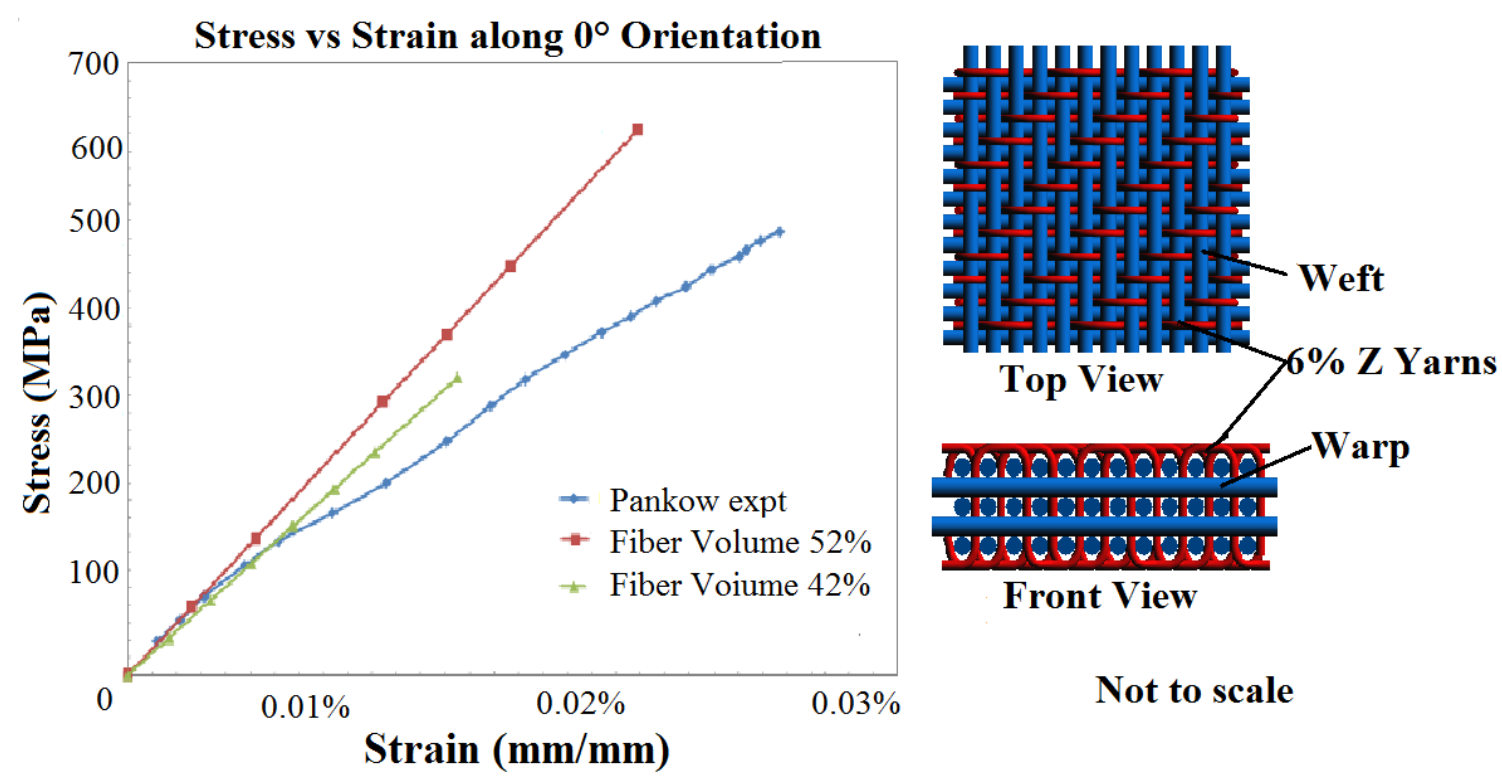

Front View

Not to scale

Figure 22 Stress-strain graph for $6 \% \mathrm{Z}$ yarns at $0^{\circ}$ orientation of the fibers

Figure 22 shows the results for $6 \% \mathrm{Z}$ yarn loaded along $0^{\circ}$ and $52 \%$ fiber volume ratio, the Young's Modulus was found to be $29.85 \mathrm{GPa}$. For $42 \%$ fiber volume ratio, the Young's Modulus was found to be $25.19 \mathrm{GPa}$. Experimental tests at $46 \%$ fiber volume ratio report a value of $24.54+/-1.42 \mathrm{GPa}$.

Figure 23 shows the results for $10 \% \mathrm{Z}$ configurations loaded along $0^{\circ}$ and $42 \%$ fiber volume ratio. This resulted in a Young's Modulus value of 14.07 GPa. Experimental tests at $45 \%$ fiber volume ratio report a corresponding value of $17.75+/-0.2757^{22}$. Similar analyses are performed along $90^{\circ}$ orientation for both $6 \%$ and $10 \% \mathrm{Z}$ configurations. For a 42\% Fiber volume fraction, simulations result in a bulk Young's Modulus of 14.08 GPa for $10 \% \mathrm{Z}$ configuration. Experimental values from literature for $45 \%$ fiber volume ratio yielded values of 21.46 +/- 1.10 GPa and 16.28 +/- 1.24 GPa respectively.

Analysis performed on $6 \% \mathrm{Z}$ configuration with $42 \%$ fiber volume fraction resulted in Young's modulus of 5.21 GPa, whereas experimental results from literature for 45\% fiber 
volume fraction yielded a value of $7.47+/-0.027 \mathrm{GPa}$. Since the simulation results are comparable to the experimental results, we proceed to the next phase of research.
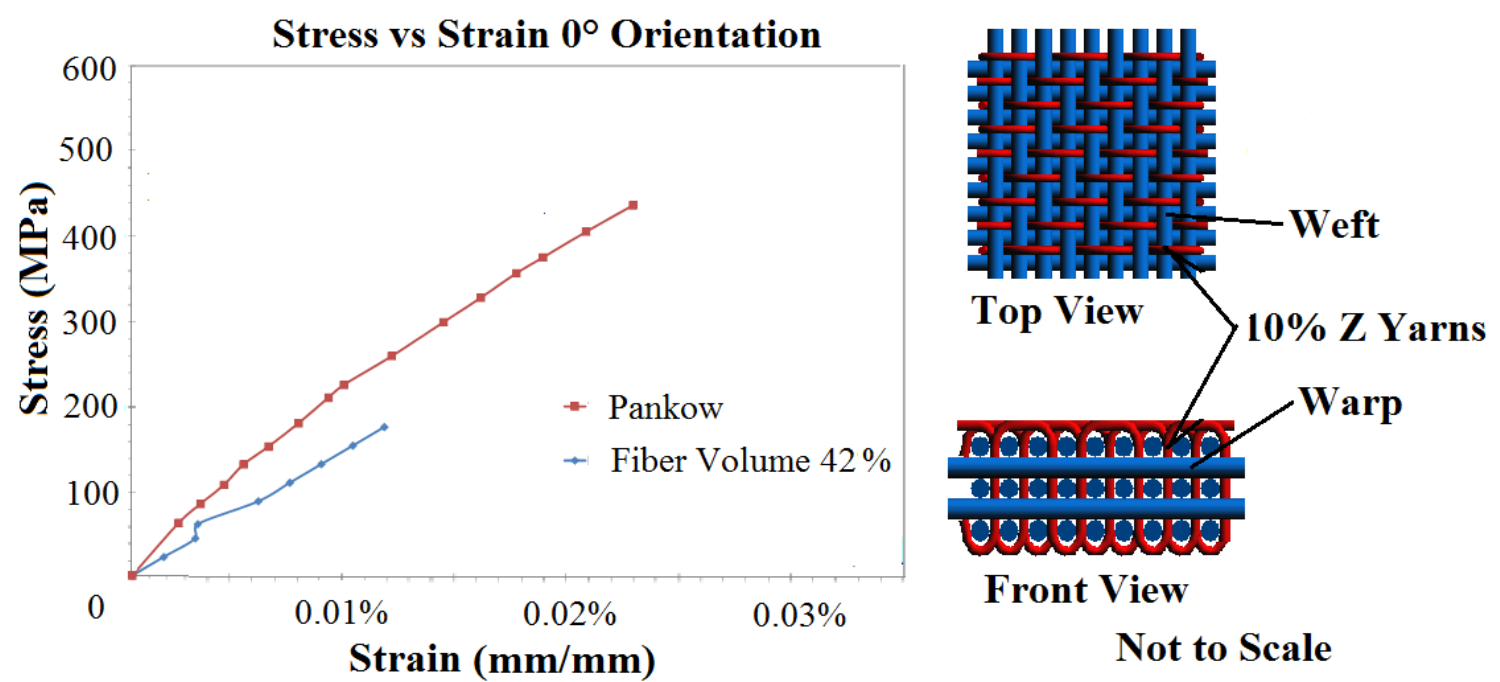

Figure 23 Stress-strain graph for $10 \% \mathrm{Z}$ yarns at $0^{\circ}$ orientation of the fibers

\subsection{Extended Finite Element based Modeling}

Several researchers reported literature on FEA based micro-analysis of 3DWC's for basic and symmetric loading conditions [10], [13], [63], [72-74]. Some of the works use both FEA and experimental testing to validate their numerical results. Since there are endless possibilities in varying the architecture and/or constituents of these woven composites, it is not feasible to perform experimental tests for all the configurations. Physics based models representing the compacted fiber and resin structures were proven to be more reliable than idealistic models. Hence in the present study, a customized software Virtual Textile Morphology Suite (VTMS) developed using physics based methods are established as a standard; to determine the realistic geometry of fibers and matrix. 
In order to determine bulk material properties of 3DWC's, another customized software B-Spline Analysis Method (BSAM) is used. Both VTMS and BSAM are developed by U.S. Air Force Research Laboratory and U.S. Army Research Laboratory in collaboration with researchers at University of Daytona and Arizona State University [64] [65] [70] [72].

The general procedure used for analysis involves:

Developing predefined 3D orthogonal textile morphologies [71] in Textile Module of VTMS software. These morphologies are modified - extended, moved and rotated as required. These fiber morphologies are then virtually relaxed and compacted in Relaxation Module to replicate the final manufactured sample. The method of multidigital chains is employed [72] to achieve this. This step generates realistic tow morphologies resulting from dry-fiber processing techniques - e.g., vacuum bag and/or platen compaction method [71]. After relaxing and compacting the fibers to the desired specifications, Tow Modify Module wraps a smooth surface (layer) around yarns (bundle of fibers). These tow surfaces are then manipulated either manually and/or automatically to remove interpenetration errors between them. These pre-formed surfaces are then clipped and/or cut to appropriate dimensions as needed. The resulting compacted preformed surfaces are used to generate tetrahedral mesh, which is compatible with BSAM. Several meshing controls are available to adjust the quality of the mesh, which follows the same meshing rules of Abaqus. Rectangular volume mesh is generated to incorporate the matrix and interface regions. The modeling process is briefly illustrated in Figure 24 (steps 1 through 6).These meshed parts are assembled in BSAM module, Boolean operations are performed to remove excess matrix material overlapping with fibers. 
Material properties for fiber and matrix are assigned along with the boundary conditions and XFEA is performed.

1) Develop Fiber morphology.

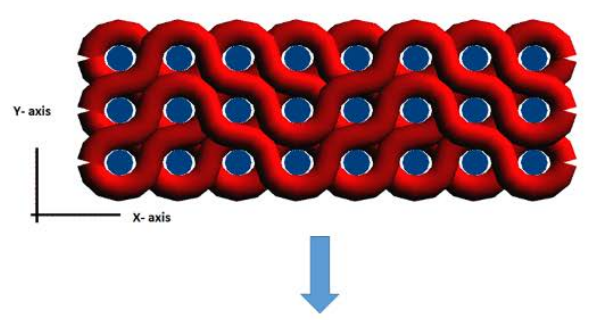

2) Generate Fiberized model.

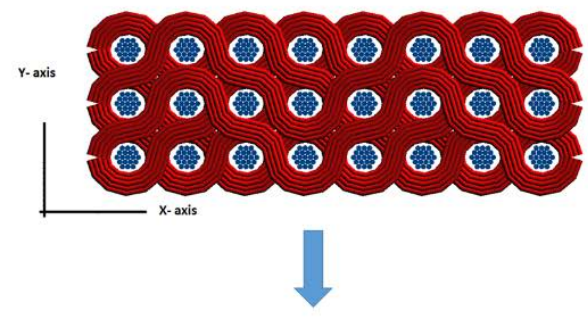

3) Generate virtually relaxed and compacted Fiber model.

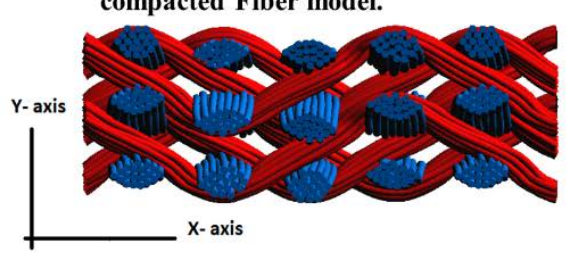

6) Add matrix/resin around and between the fiber interfaces.

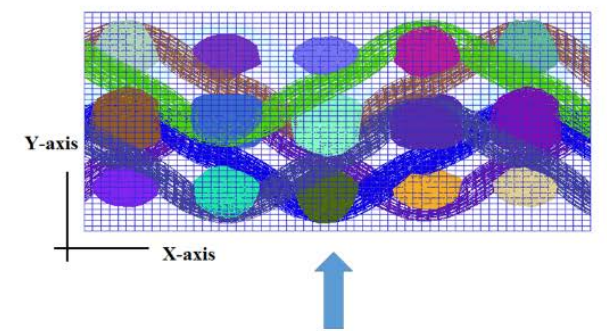

5) Generate Finite Elemental Mesh for Fibers from yarn model.

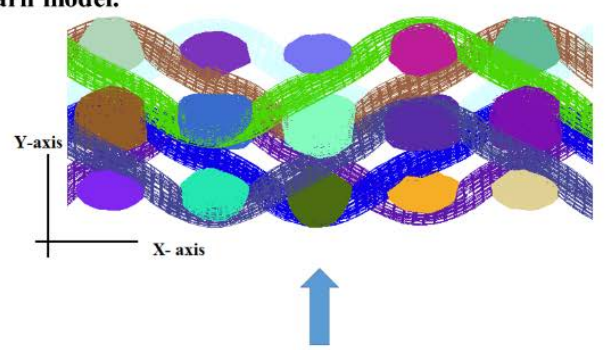

4) Generate yarn model and remove

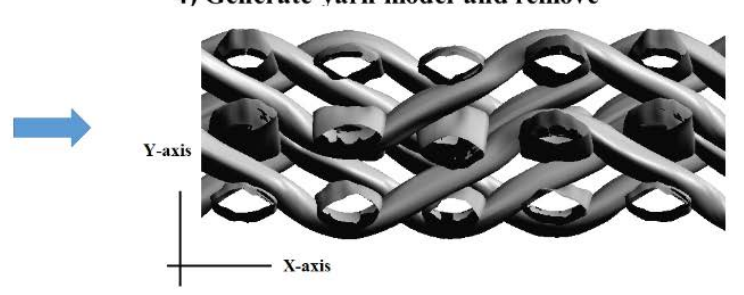

Figure 24 General steps involved in developing 3D Woven Composite Model

\subsection{Architecture selection for micro-analysis}

Four different predefined architectures are considered in this study given as: 3D Orthogonal Woven (3DOW), Ply to Ply Inter-lock without Warp (P2PILwoW), Ply to Ply Angle Inter-lock without Warp (P2PAngILwoW) and 2D Tri-axial Braided (Tri-Axial) structure. For the 3D Orthogonal Woven architecture, two different Z percent fiber volumes of $6 \%$ and $10 \%$ were considered. For ease of readability, all the 4 architectures are named as architecture 1 , architecture 2, architecture 3 and architecture 4 respectively. 
A detailed explanation of the methodology developed for analysis of Architecture 1 is given in the next section followed by a brief explanation of the other 3 architectures.

\subsubsection{Development of 3D Orthogonal Woven Structure (3DOW)}

Predefined 3DOW morphologies are developed in textile module of VTMS. The specifications used for one of the models are as follows: a diameter $0.96 \mathrm{~mm}$ for warp and weft, a diameter of $0.48 \mathrm{~mm}$ for z-yarns with a tow spacing of $1.92 \mathrm{~mm}$, binder pattern 2x1 with 2 layers repeated twice in $\mathrm{X}$ and $\mathrm{Z}$ directions. The layout with brief labelling is shown in Figure 25. The developed model is extended 2 units on all ends to make compaction process smoother and avoid edge factors. A sub- yarn topology is generated with 5 sub-yarns in all directions. This will create a bundle of 19 digital fibers representing each yarn. These morphologies are moved as needed to shift one corner of the morphology to origin and are generally called as sub-yarn morphologies. These specifications yielded a total of $42 \%$ fiber volume along warp and weft together and $10 \%$ fiber volume along z-yarns. 


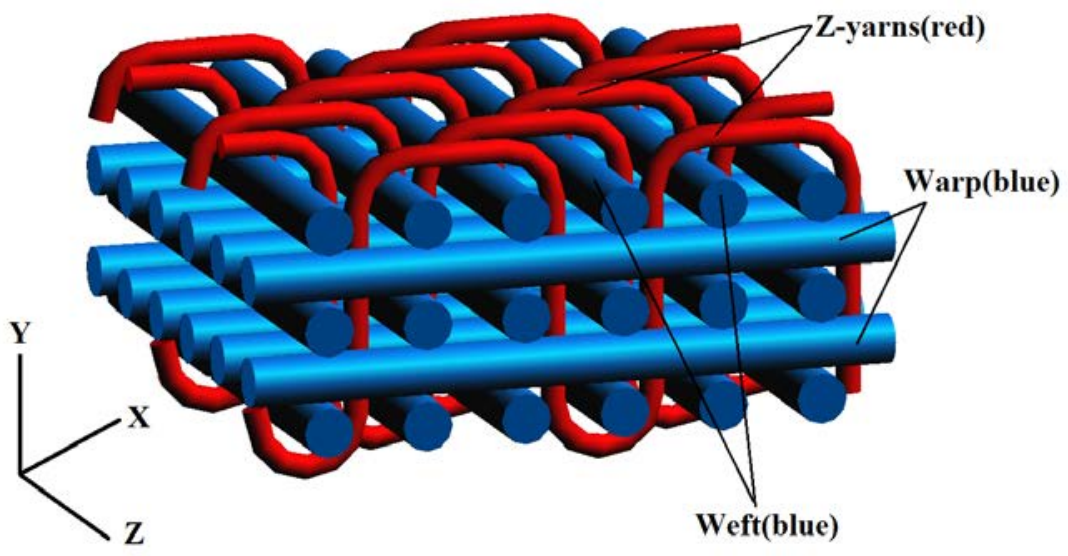

Figure 25 3D Orthogonal Woven

These sub-yarn morphologies are relaxed in Relaxation Module for 1 cycle using a damping value of 100 and the fibers are loaded using edge fixed condition. This morphology is further relaxed for 3 cycles using a damping value of 100 and fibers are loaded allowing 5 to $10 \%$ of edge pressing. The relaxation process is further continued with relaxation parameters of 15 cycles at damping value of 100 and fibers in edge fixed condition.

These relaxed sub-yarn morphologies are further modified in Textile Module. A pair of mold is created just above and below the model, which represent the platten or vacuum bag in dry-fiber processing technique.

The relaxed model and the two molds are assembled in Relaxation Module. Generic material properties are assigned for $\mathrm{E}_{11}$ (longitudinal). Values for $\mathrm{E}_{11}$ range from 0.01 to 1.0 (representing relative values). Same values for all the parts in the model will lead the model to smear uniformly. If different materials are used for fibers i.e., different materials for warp, weft and binder, different values of $\mathrm{E}_{11}$ are assigned relatively based on their strengths. The relaxation parameters for fibers are set to 4 cycles with a damping 
value of 25 and edges fixed. This time, the mold compaction is turned on with top mold rigid and loading the bottom mold to $0.05 \mathrm{~mm}$. After relaxing once, the mold compaction settings are modified changing bottom mold to rigid and top mold to $-0.5 \mathrm{~mm}$. Thus, for every relaxation cycle, the top mold moves down $0.5 \mathrm{~mm}$ thereby pressing or compacting the fibers. The above relaxation cycle is repeated until the desired thickness is achieved, which reflects the thickness obtained if a sample was actually manufactured.

A surface is generated from each fiber/yarn model after relaxation and wraps around the smeared yarn and is called a tow. The tows are generated by discretizing the model to several slices and stacks. All the other tows are generated using a similar approach by opening one fiber at a time. These tows are further re-meshed by adjusting the number of slices and stacks. If the tows are sufficiently long, it is recommended to create 28 to 32 slices and 50 to 60 stacks. For very low tow lengths, it is recommended to use lower values for slices and stacks. The re-meshing is needed to further smooth the rough surfaces.

The newly generated smooth tows are re-assembled in Tow Modify Module and an extra length of 1 or 2 units are added to each tow. This will reduce the errors caused due to tangency at the edges during meshing. These tow surfaces are checked for interpenetrations. The interpenetrated surfaces are manipulated either manually and/or automatically to remove interpenetrations. These preform surfaces are then clipped and/or cut to appropriate size as needed, which represent a unit Representative Elementary Volume (REV).

In Mesh Generator Module, these compacted tows representing fibers are used to generate hexahedral meshes (C3D8). Several mesh control parameters - medial axis 
effect, mesh density, coarse to fine cross wire and other settings are adjusted to achieve mesh without bad elements. The quality of the mesh is determined using the same meshing rules in Abaqus $\backslash$ Simulia 3DS i.e., considering aspect ratio and skewness of elements to determine the quality of the mesh. A rectangular volume mesh, enclosing these fibers/tows is generated, which represents the resin or matrix of the composite. BSAM uses Independent Mesh Method (IMM) to define the interface elements see Figure 26(b), where the tow elements intersect matrix elements. If this same model is imported to Abaqus CAE, it has no way to handle this interface regions. These regions need to be manually connected, which is very difficult and error prone especially given the small weird tow and resin pockets [70].

a)

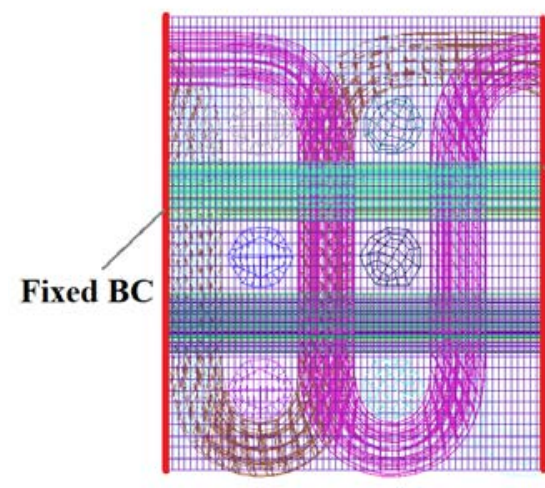

b)

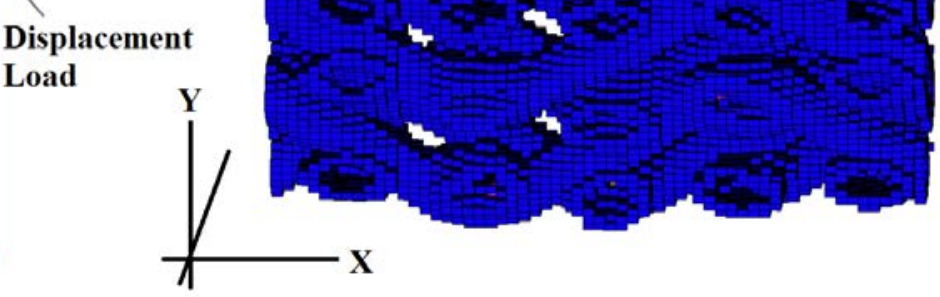

Figure 26- a) 3DOWC Meshed model with Boundary Conditions b) P2PILwoW showing Interface elements - connection of tow and resin elements.

These meshed parts are assembled together in BSAM Module to perform Finite Elemental Analysis. A set of materials are selected for fiber and matrix and their corresponding mechanical properties are assigned to the imported model. Fixed and 
displacement Boundary Conditions are applied to chosen surfaces as shown in Figure 26(a) and then XFEA analysis is performed using BSAM. Upon convergence of the analysis, the resulting values of principal stress and principal strain are extracted from the output and are plotted. Similar procedure is followed to build the other three architectures.

A new 3DOW model with the same/similar architecture but with $10 \%$ volumes in z-yarns is considered. The specifications used for this model are: diameter of $0.86 \mathrm{~mm}$ for warp and weft, a diameter of $0.4 \mathrm{~mm}$ for z-yarns with a tow spacing of $1.72 \mathrm{~mm}$, a binder pattern 2x1 with 2 layers. This configuration yielded a model with a total of 42 percent fiber volume along warp and weft together and 6 percent fiber volume along z- yarns.

\subsubsection{Development of P2PIL}

A predefined 3D Ply to Ply Interlock without warp (P2PILwoW) with a weft diameter of $0.5 \mathrm{~mm}$, a spacing of $0.75 \mathrm{~mm}$, binder (z-yarn) diameter of $0.2 \mathrm{~mm}$ with a spacing of $0.25 \mathrm{~mm}$ with 2 layers and repeated twice in $\mathrm{X}$ and $\mathrm{Z}$ directions, is shown in Figure 27.

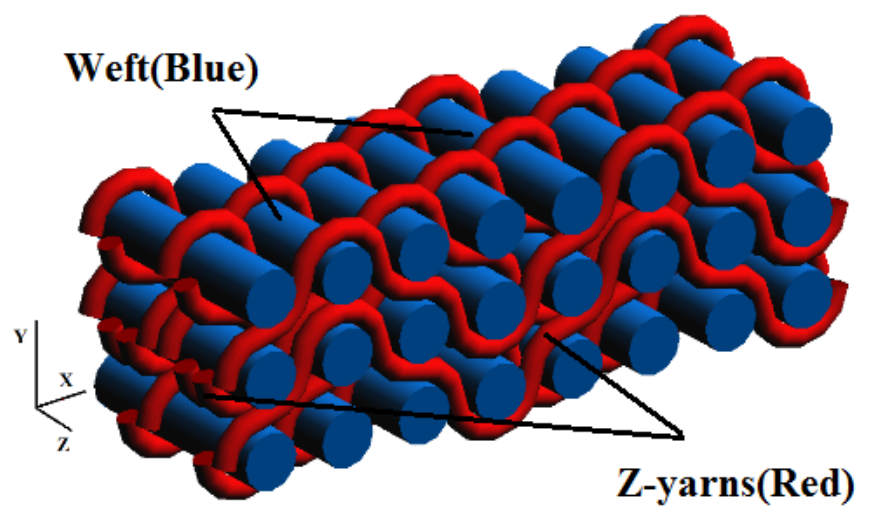

Figure 27 Ply to Ply Inter- Lock without Warp 


\subsubsection{Development of P2PAng}

A predefined 3D Ply to Ply Angle Inter-Lock without warp (P2PAngILwoW) with a weft diameter of $0.5 \mathrm{~mm}$ and a spacing of $0.75 \mathrm{~mm}$, binder (z-yarn) diameter of $0.2 \mathrm{~mm}$ with a spacing of $0.25 \mathrm{~mm}$ with 2 layers and repeated twice in $\mathrm{X}$ and $\mathrm{Z}$ directions is shown in Figure 28.

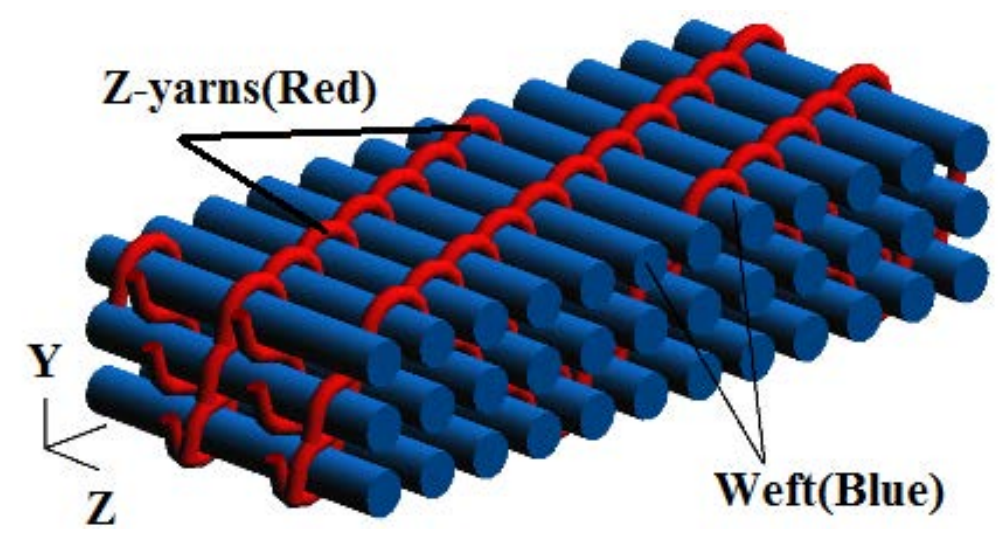

Figure 28 Ply to Ply Angle Inter- Lock without Warp

\subsubsection{Triaxial}

It is the predefined Triaxial braided morphology with an axial diameter of $0.5 \mathrm{~mm}$ with braids of diameter $0.25 \mathrm{~mm}$ at a braiding angle of $45^{\circ}$ and unit cell width of $1.5 \mathrm{~mm}$ and repeated four times in both $\mathrm{X}$ and $\mathrm{Z}$ directions and is as shown in Figure 29. 


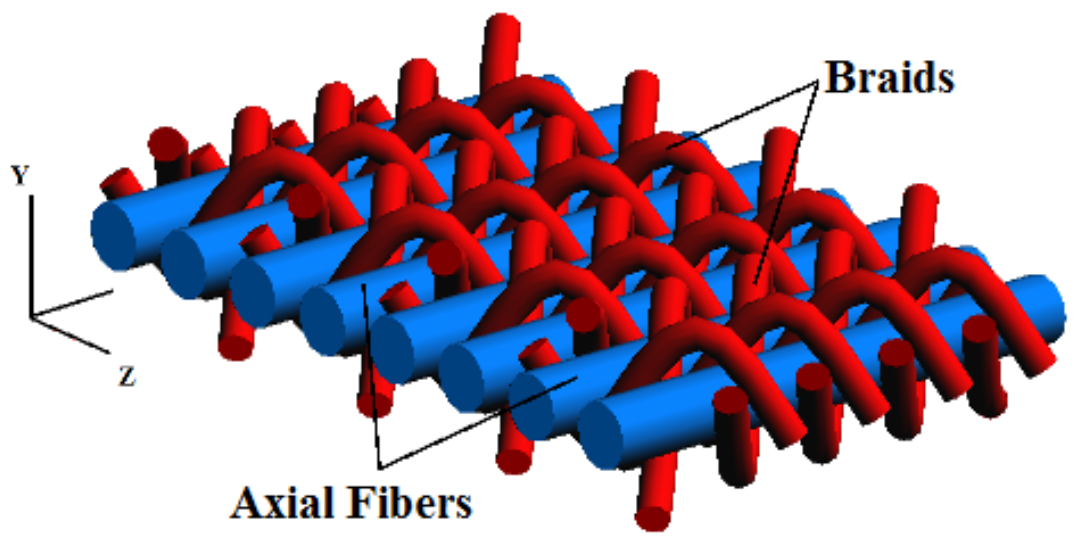

Figure 29 Tri-axial Braided

\subsection{Material Selection for the micro-analysis}

The most common hip implant materials are metals and metallic alloys. Apart from metals, other materials considered are ceramics, cements and unidirectional polymeric fibers. In the present work, several of these and other materials used for hip implants were studied to determine the positive and negative aspects. Three fiber materials namely Ti6Al4V, which is medical grade 5, CoCr alloy and Carbon Fiber along with two polymer materials UHMWPE and PEEK are considered. These polymers are all biocompatible and widely used in medical implants. The material models for each constituent used in the analysis are given in Table 4.

Table 4 Mechanical Properties of fibers and resin.

Fibers are assumed to be transversely isotropic which gives the following assumption:

$$
\left(E_{33}{ }^{\mathrm{f}}=\mathrm{E}_{22}{ }^{\mathrm{f}}, \mathrm{G}_{13}{ }^{\mathrm{f}}=\mathrm{G}_{12}{ }^{\mathrm{f}} \text { and } \mathrm{G}_{23}{ }^{\mathrm{f}}=\mathrm{E}_{22}{ }^{\mathrm{f}} / 2\left(1-v_{23}{ }^{\mathrm{f}}\right)\right)
$$

\begin{tabular}{ccccccc}
\hline Fibers & $\mathbf{E}_{\mathbf{1 1}}$ & $\mathbf{E}_{\mathbf{2 2}}$ & $\mathbf{G}_{\mathbf{1 2}}$ & $\mathbf{X}_{\mathbf{t}}$ & $\mathbf{v}_{\mathbf{1 2}}$ & $\boldsymbol{\rho}(\mathbf{g m} / \mathbf{c c})$ \\
\hline Carbon Fiber(Hexcel) & 231 & 50.2 & 50 & 4150 & 0.1 & 1.78 \\
Ti6Al4V & 114 & 11.4 & 44 & 1100 & 0.3 & 4.43 \\
CoCr Alloy & 220 & 22 & 45 & 4500 & 0.3 & 4.5 \\
\hline
\end{tabular}




\begin{tabular}{ccccc}
\hline Resin & $\mathbf{E}_{\mathbf{1 1}}$ & $\mathbf{X}_{\mathbf{t}}$ & $\mathbf{v}_{\mathbf{1 2}}$ & $\boldsymbol{\rho}(\mathbf{g m} / \mathbf{c c})$ \\
\hline UHMWPE & 0.75 & 30 & 0.46 & 0.93 \\
PEEK-HA & 3.59 & 97.2 & 0.4 & 1.3 \\
\hline
\end{tabular}

$\mathrm{E}_{11}, \mathrm{E}_{22}, \mathrm{E}_{33}$ - elastic Modulus (GPa) in X, Y and Z coordinate system.

$\mathrm{G}_{12}, \mathrm{G}_{13}, \mathrm{G}_{23}$ - Shear Modulus (GPa) in XY, XZ and YZ planes.

$\mathrm{X}_{\mathrm{t}}$ - Tensile strength (MPa) $\quad v$ - poisson's ratio $\quad \rho$-density of material ${ }^{\mathrm{f}}$ - fiber 


\section{CHAPTER 5-Finite Element Analysis of Hip Implant}

In this chapter details of developing hip implant model, meshing strategies, applied materials and loading conditions used to analyze the model are discussed. SolidWorks ${ }^{\circledR}$ 2013(Dassault Systemes SolidWorks Corporation, Concord, MA, USA) is used to create the hip implant models with different design variables. A baseline hip implant is designed and modified to develop three different hip models using both design and non-design parameters. These solid models are imported to the finite element preprocessor - HyperMesh V13.0 (Hyperworks, Altair Engineering, Troy, MI, USA) to develop the needed orphan mesh, create sets, sections and assign material properties to these sections. The output from HyperMesh, is as an input file required to perform robust FEA solver in Abaqus.

\subsection{Development of solid hip implant model}

A baseline solid hip implant model with stem and head are developed in SolidWorks. SolidWorks is widely used to create complex mechanical models. First the major solid component femoral stem of the hip implant was created. A stem model from literature was chosen to generate the required dimensions for designing a baseline model [75]. Overall stem dimensions are approximately $18 \mathrm{~cm}$ in height, including the neck length of approximately $2 \mathrm{~cm}$. Neck diameter, neck angle and other factors are not changed to avoid increase in variations (independent parameters). The length and distance parameters are kept constant, changing only the cross section of the profile as shown in Figure 30(b). These two baseline implant models are assigned traditional material properties i.e., isotropic Ti6Al4V alloy for stem and COCr alloy for the head. To consider 
functionally graded stem, these two base models are further discretized to number of subsections (say 16 or 17) and each section is assigned material properties which mimic the functionally graded material.

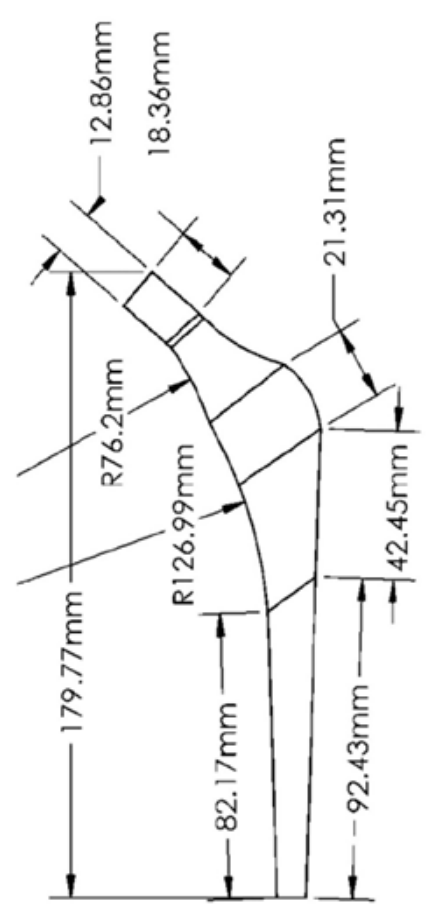

a)

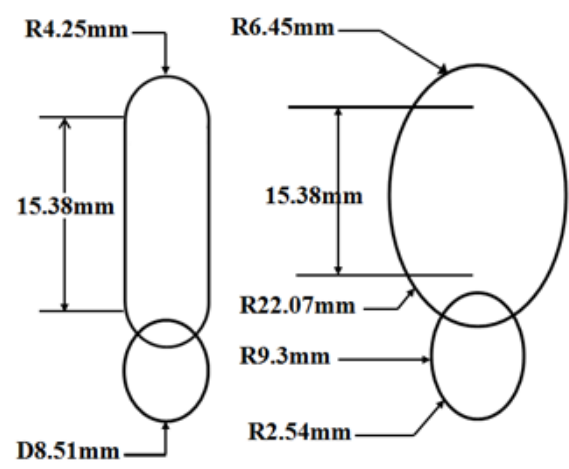

b)

Figure 30 Stem dimensions a) lengthwise b) cross-section profiles [75]

A femoral head was designed as a basic model with a nominal diameter of $40 \mathrm{~mm}$ with a cylindrical cut to allow stem neck insertion. These two components are assembled together as shown in Figure 31(a). Femoral head is fitted to the top of the neck and aligned for proper fixation. 


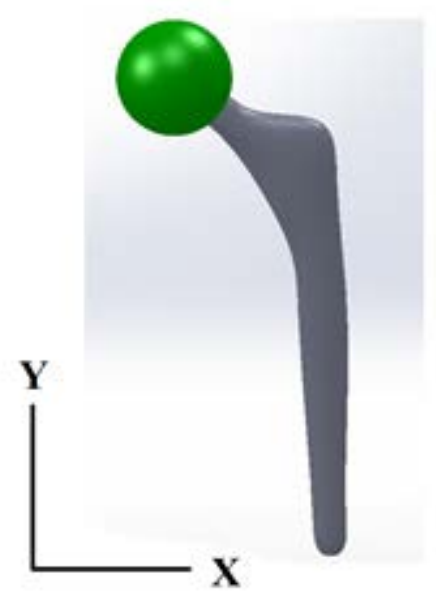

a)

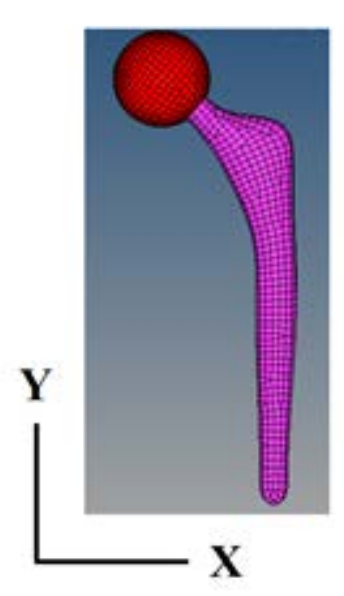

b)

Figure 31 (a) Assembly of Hip Implant in SolidWorks (b) Finite Element Mesh

\subsection{Preparation for solid processing into HyperMesh}

All the solid models created in SolidWorks are exported to both iges and step (.stp) file formats. These file formats are neutral files and can be easily imported by Hypermesh. Step file format provided higher accuracy due to the complex geometry of the implant assembly. Iges file format was not used for analysis due to failure of importing all geometries.

\subsubsection{Preprocessing of finite element model}

Hypermesh FEA tool/software is a reliable software widely used in industrial, automotive, biomedical and aerospace fields. This software is more predominantly used as a preprocessor to create a file which is compatible to be used in a much complex FEA solvers like Abaqus. FEM converts a complex geometry into sub-divisions called finite

elements. The geometrical elements collectively represent an entire geometry. The size of 
these elements is usually selected by user for preferred accuracy of solution. Course and fine meshing discretizes the entire model into larger or smaller elements respectively. Consecutive elements in the same geometry are connected by common points called nodes. Several nodes are connected to construct an element and several elements combined together will form a complex geometry. When boundary conditions are applied, the interaction between nodes will result in nodal solution whereas the interactions between elements created by nodal interfaces will generate elemental solution. The range of motion for elements is the function of permitted nodal vector constraints, called degrees of freedom (DOF) [76].

Two different stems are modeled using different cross sections. These two models are assigned traditional material properties i.e, isotropic Ti6Al4U Grad 5 alloy for the stem and CoCr alloy for the head. To consider functionally graded stem, these stem model is discretized into numbers of sub section (say 16 or 17) and each section is assigned functionally varying material property. The classification of these models are detailed in Table 5.

Table 5 Details of different implant models

\begin{tabular}{|c|c|c|}
\hline IMPLANT \# & Profile of stem & FG Material ( Yes/ No) \\
\hline 1 & 1 & No \\
\hline 2 & 2 & No \\
\hline 3 & 1 & Yes \\
\hline 4 & 2 & Yes \\
\hline
\end{tabular}




\subsubsection{Meshing and mesh refinement}

The neutral hip implant assemblies are imported to Hyper Mesh 13.0. After importing the geometries successfully, the geometries are cleaned up using target element size and auto clean option. 2D membrane elements of $2 \times 10^{-4} \mathrm{~mm}$ thickness are created on the surface of the implant model using mixed first order triangular (M3D3) and quadrilateral (M3D4) elements as shown in Figure 31(b). These membrane elements are used to generate $2^{\text {nd }}$ order 3D tetrahedral elements (C3D10I) as shown in Figure 32Figure 33.

Even though hexahedral elements are preferred for their accuracy, due to the complexity of the implant geometry, a $2^{\text {nd }}$ order tetrahedral elements are considered for this study.

Initial target element size of 5 is considered based on the overall size of the model and the entire model is meshed. This resulted in a coarser mesh as shown in Figure 32.

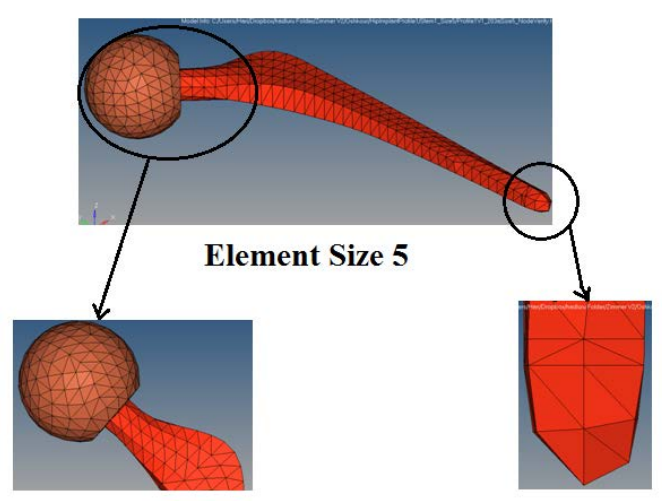

Element Size 5 - Zoomed

Figure 32 Implant 1 with target element size 5 
Different target element sizes 4, 3, 2 and 1 are considered and the FEA analysis is performed to determine the final results, which are mesh independent. Implant 1 with target element size 1 is shown in Figure 33. Different target element size and their corresponding number of 2D and 3D nodes and elements are listed in Table 6 Table 7 for Implant 1 and Implant 2.

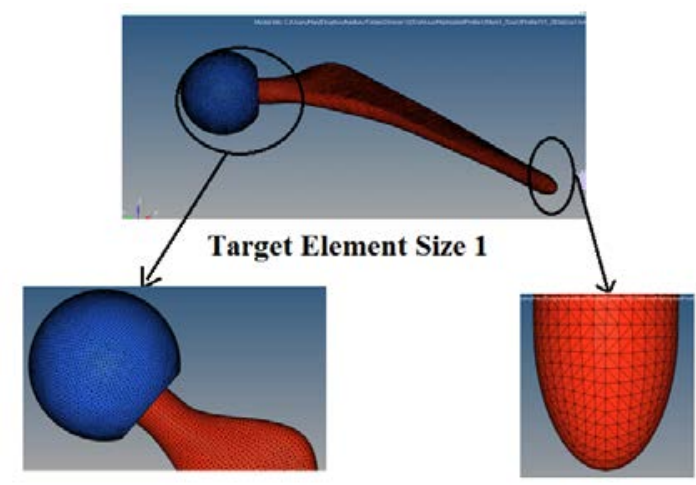

Element Size 1- Zoomed

Figure 33 Implant 1 with target element size 1 
Table 6 Implant 1 with target element size, number of nodes and elements

\begin{tabular}{|c|c|c|c|c|c|c|c|}
\hline Target & Implant & \multicolumn{2}{|c|}{ 2D Membranes } & \multicolumn{2}{c|}{ 3D Solids } & \multicolumn{2}{c|}{ Total } \\
\cline { 3 - 8 } $\begin{array}{c}\text { Element } \\
\text { Size }\end{array}$ & 1 & Nodes & Elements & Nodes & Elements & Nodes & $\begin{array}{c}\text { Eleme- } \\
\text { nts }\end{array}$ \\
\hline \multirow{2}{*}{5} & Stem & 346 & 352 & 361 & 861 & 1305 & 2732 \\
& Head & 251 & 269 & 347 & 1250 & & \\
\hline \multirow{2}{*}{4} & Stem & 745 & 780 & 881 & 2664 & 2558 & 5965 \\
& Head & 375 & 400 & 557 & 2121 & & \\
\hline \multirow{2}{*}{3} & Stem & 1078 & 1110 & 1361 & 4469 & 4340 & 11225 \\
& Head & 705 & 748 & 1196 & 4898 & & \\
\hline \multirow{2}{*}{2} & Stem & 2523 & 2570 & 3839 & 14754 & 10711 & 31406 \\
& Head & 1474 & 1523 & 2875 & 12559 & & \\
\hline \multirow{2}{*}{1} & Stem & 8926 & 9023 & 17368 & 75840 & \multirow{2}{*}{47948} & 163300 \\
& Head & 6335 & 6430 & 15319 & 72007 & & \\
\hline
\end{tabular}

Table 7 Implant 2 with target element size, number of nodes and elements

\begin{tabular}{|c|c|c|c|c|c|c|c|}
\hline Target & Implant & \multicolumn{2}{|c|}{ 2D Membranes } & \multicolumn{2}{c|}{ 3D Solids } & \multicolumn{2}{|c|}{ Total } \\
\cline { 3 - 8 } $\begin{array}{c}\text { Element } \\
\text { Size }\end{array}$ & 2 & Nodes & Elements & Nodes & Elements & Nodes & Elements \\
\hline \multirow{2}{*}{5} & Stem & 422 & 442 & 2715 & 1334 & 3905 & 3942 \\
& Head & 298 & 322 & 470 & 1844 & & \\
\hline \multirow{2}{*}{4} & Stem & 594 & 611 & 4097 & 2098 & \multirow{2}{*}{5397} & 5714 \\
& Head & 418 & 442 & 4288 & 2563 & & \\
\hline \multirow{2}{*}{3} & Stem & 1093 & 1120 & 9349 & 5261 & 12314 & 11943 \\
& Head & 693 & 722 & 1179 & 4840 & & \\
\hline \multirow{2}{*}{2} & Stem & 2503 & 2557 & 3971 & 15600 & 11332 & 34060 \\
& Head & 1626 & 1673 & 3232 & 14230 & & \\
\hline \multirow{2}{*}{1} & Stem & 9191 & 9285 & 18532 & 81855 & \multirow{2}{*}{169670} \\
& Head & 6355 & 6449 & 15345 & 72081 & & \\
\hline
\end{tabular}




\subsubsection{Material properties}

For baseline models (Implant 1 and 2), linear elastic material properties are considered for stem and head, which are shown in Table 8

Table 8 Material properties for stem and head

\begin{tabular}{ccccc}
\hline Part & Material & $\mathbf{E}(\mathbf{G P a})$ & $\mathbf{v}_{\mathbf{1 2}}$ & $\boldsymbol{\rho}(\mathbf{g m} / \mathbf{c c})$ \\
\hline Stem & Ti6Al4V & 120 & 0.342 & 4.43 \\
Head & CoCr Alloy & 230 & 0.3 & 8.8 \\
\hline
\end{tabular}

For functionally graded material the elastic modulus of the stem is varied considering a power law. From previous micro analysis, we concluded that the modulus can be varied from distal to proximal end of the stem as needed. In this study an elastic modulus of $E_{b}=20 \mathrm{GPa}$ is considered at the distal end and $E_{a}=120 \mathrm{GPa}$ is considered at the proximal end. The elastic modulus is discritized using the formula

$$
E_{k}=E_{a}\left(\frac{i}{t o t}\right)^{n}+E_{b}\left[1-\left(\frac{i}{t o t}\right)^{n}\right]
$$

where $\mathrm{n}$ takes a value between 0 to 1.0 .

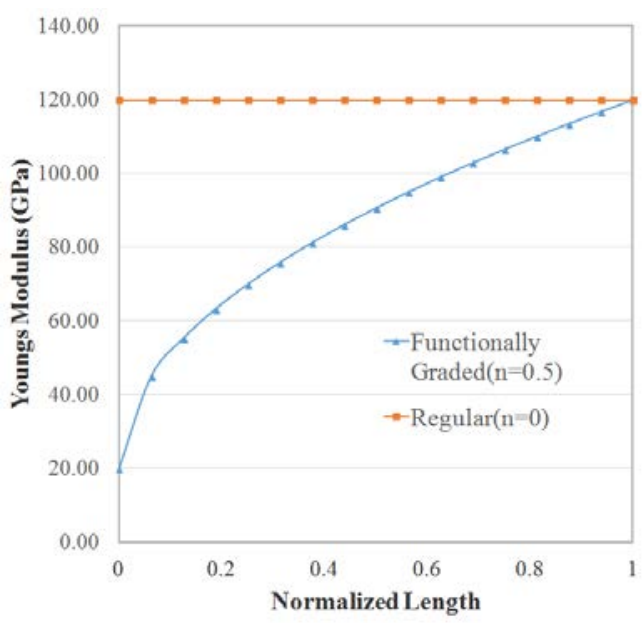

Figure 34 Young's modulus for base model and functionally graded model w.r.t length 
For this analysis we considered a value of $\mathrm{n}=0.5$ and stem is discretized to 17 parts. The values of $E$ along the length of the stem are shown in Figure 34. The sections made in the stem to assign different material properties is shown in Figure 35.

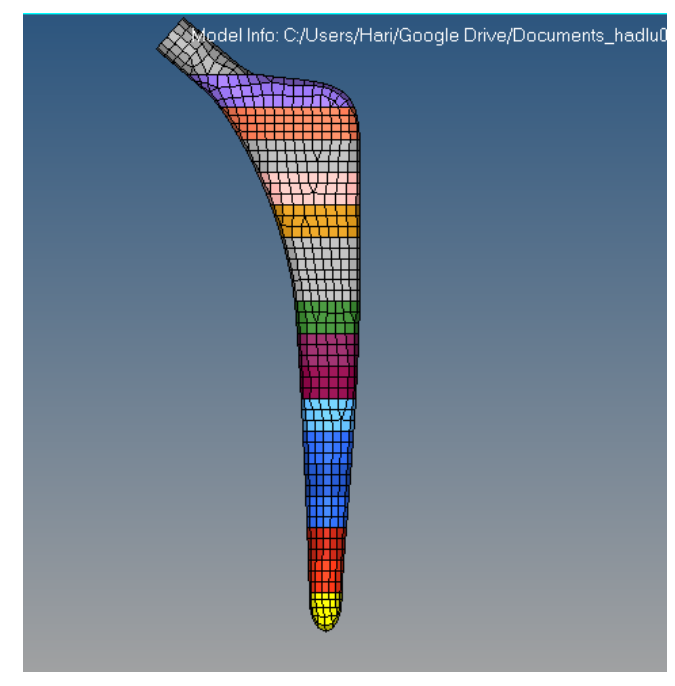

Figure 35 Stem showing functionally graded material sections.

\subsubsection{Defining Contact Surface}

In Abaqus, each part is on its own and are not interlinked or tied automatically. The user has to specifically define the interactions between the parts. For this analysis, a basic surface-surface based tie constraint is specified between the head and stem parts with a tolerance of $0.5 \mathrm{~mm}$. This means any surfaces, which are within a distance of $0.5 \mathrm{~mm}$ are considered to be tied together.

\subsubsection{Boundary and load conditions}

Three different sets, two nodal sets and one element set are predefined to apply appropriate boundary and load conditions. 
Assuming the bone ingrowth already occurred near the porous surface of the stem, the nodes near distal end (marked A) are totally fixed i.e., both translational (U1, U2 and U3) and rotational (U4, U5 and U6) are totally constrained. Also another location (marked B) shown in Figure 36 is pinned meaning stem is not constrained rotationally but is fixed translationally.

We also incorporated into the model the mechanical behavior of a trunnion-head junction simulating the load experienced by the head during the single-leg support phase of normal level walking. This corresponded to a pressure load of $2.1 \mathrm{MPa}$, which is applied to the dome of the head as shown in Figure 36 which simulates 2.6 times bodyweight force at the hip [77] [78].

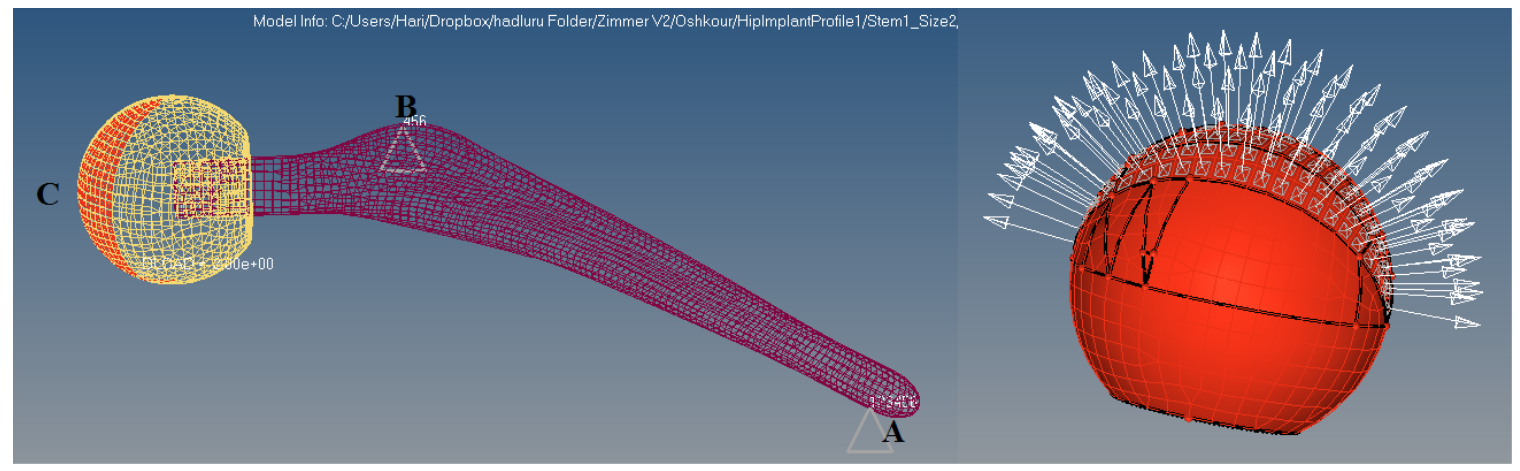

Figure 36 Implant showing boundary and loading conditions

Abaqus solver is used to perform actual FEA analysis. The results obtained are given in the next chapter. 


\section{CHAPTER 6-Results and discussion}

\subsection{Micro-analysis results}

Over each architecture, all combinations of fiber material and matrix material were compared in two directions (longitudinal and orthogonal) for a total of twelve individual modulus calculations per architecture. The first architecture, Ortho, resulted in a longitudinal modulus within the range of 15-20 GPa for each material combination. While the typical loading case for micromechanics models is to incorporate period boundary conditions, to limit the computational complexity samples were loaded by applying a fixed/displacement boundary condition on opposite surfaces corresponding to the loading direction. As the scope of the study was to determine a range of modulus to match pre-existing specifications, the error associated with the simplified boundary conditions was ignored. The stress distribution of the ortho unit cell with Ti6Al4V fibers and UHMWPE resin is shown in Figure 37. In Figure 37(b) the fibers are isolated and the stress distribution in fibers is shown. The fibers in the warp direction have average stress value of 35.7 MPa in the warps for 10\% Z-yarns and an average stress value of $31.2 \mathrm{MPa}$ for $6 \%$ Z-yarns for a displacement load of $0.008 \mathrm{~mm}$. The simulation results provide principle stress and principle strain matrices. The numerical values of the elastic modulus in the longitudinal directions are summarized in Table 3. From the table, it is evident that the elastic modulus for the combination of carbon fiber and PEEK is highest (26.81GPa) among all the combinations and hence stronger. 
a)

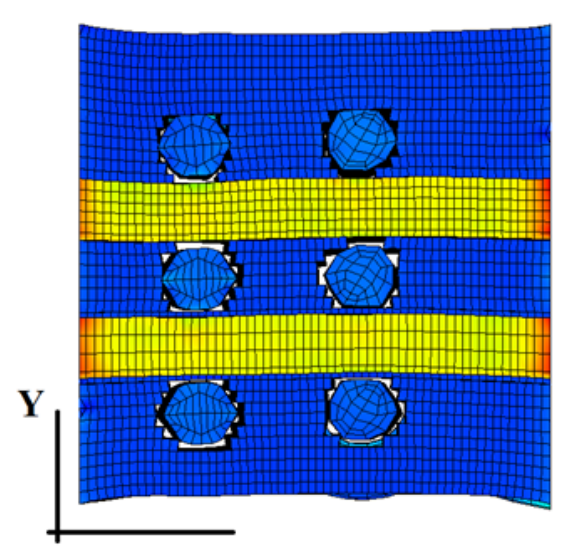

b)

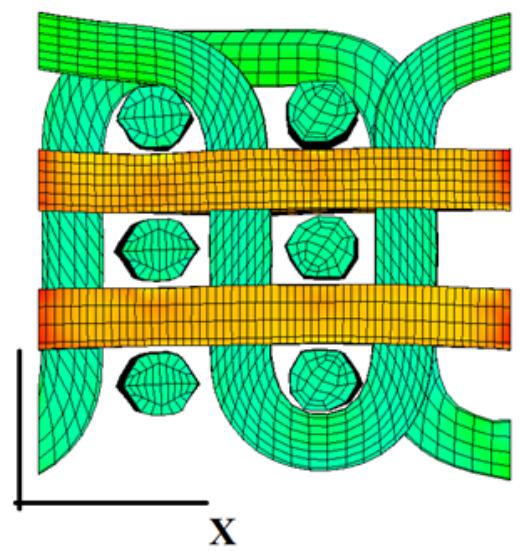

$\mathrm{S}_{\mathrm{xx}}(\mathrm{GPa})$

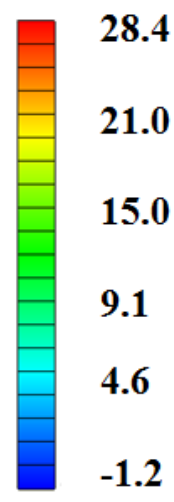

Figure 37 Stress Sxx of Ortho model - Z6\% loaded in X direction a) Unit Composite b) Only Fibers

Table 9 3D Orthogonal Woven Composites 6\% Z yarns - elastic modulus (GPa) longitudinal

\begin{tabular}{ccc}
\hline Fiber $\downarrow$ & Matrix/resin $\rightarrow$ & PHMWPE \\
\hline CF & & \\
Ti6Al4V & 23.02 & 26.81 \\
CoCr alloy & 13.62 & 15.38 \\
& 22.03 & 25.78 \\
\hline
\end{tabular}


a)

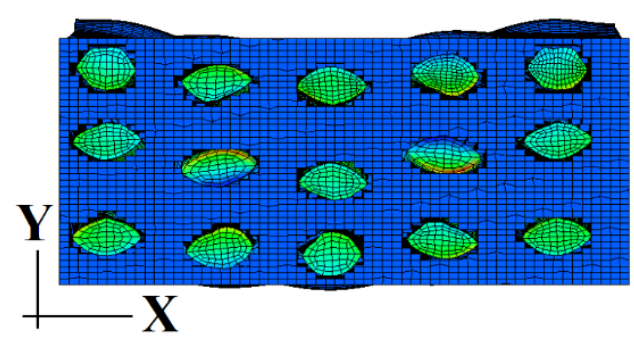

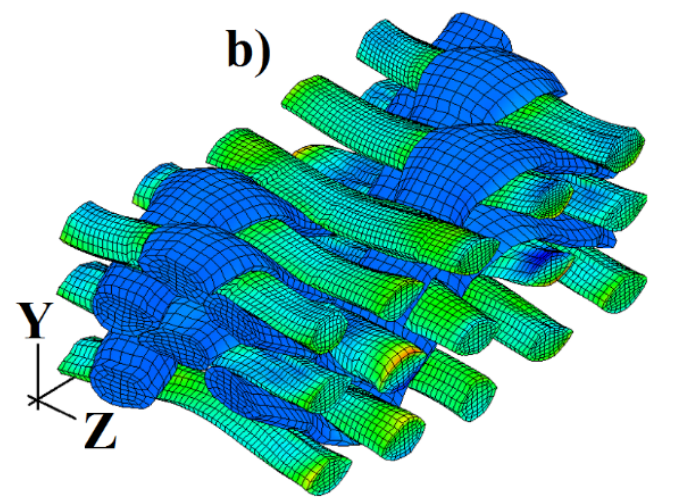

$\mathrm{S}_{\mathrm{ZZ}}$ (Gpa)

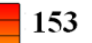

111

77.4

35.6

$-6.1$

Figure 38 Stress Szz of P2PIL loaded in Z direction (a) Unit Composite (b) Fibers only

Stress distribution of P2PIL with Carbon Fiber and PEEK as resin is shown in Figure 38 with loading in $\mathrm{Z}$ direction for both the unit cell and fiber only configurations. The fibers in the weft direction are more stressed in this load setting with average stress value of 53.5 MPa with one face fixed and other face loaded at $0.01 \mathrm{~mm}$. The simulation results provide principle stress and principle strain matrices. Stress-strain plots for various loading conditions are shown in Figure 39 and elastic modulus is estimated from these graphs. The numerical values of the elastic modulus in the longitudinal directions are summarized in Table 10

Similar analysis is performed for this architecture with loading in $\mathrm{X}$ direction. The inter-locking fibers are more stressed in this load setting with average stress value of 8.7 $\mathrm{MPa}$ with one face fixed and other face loaded at $0.01 \mathrm{~mm}$. Elastic modulus $\left(\mathrm{E}_{33}\right)$ in transverse direction are calculated from the analysis results and are summarized are in

Table 11. 

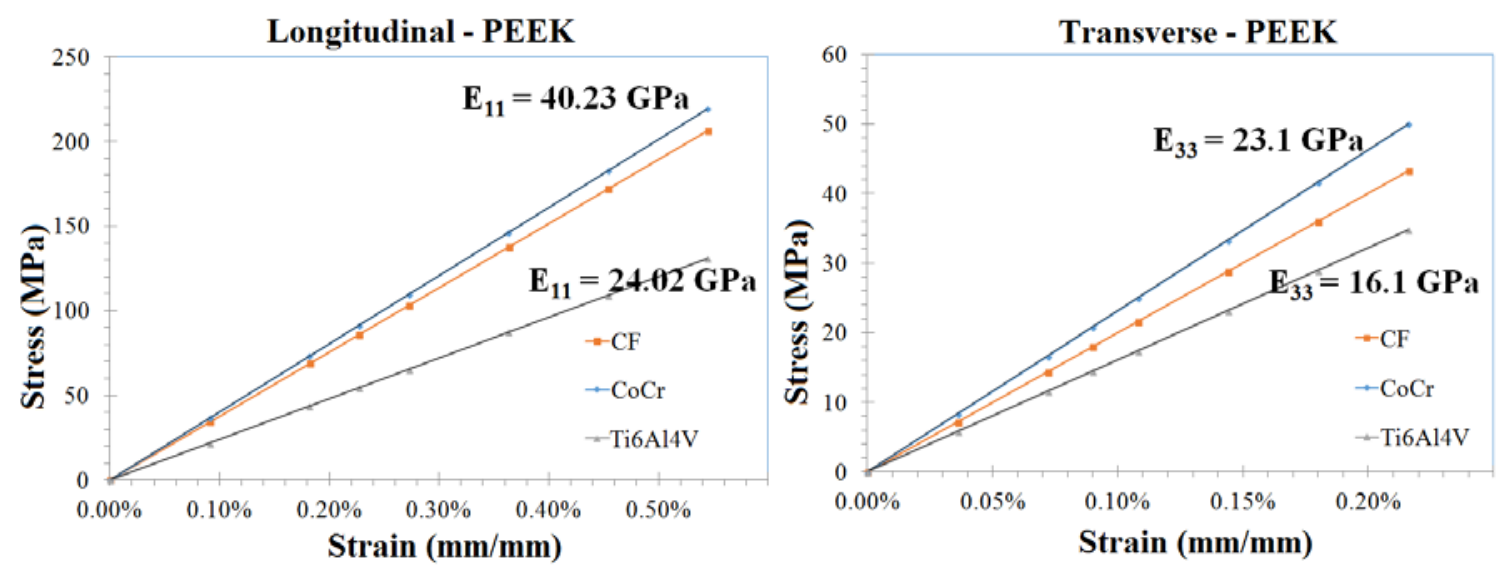

Figure 39 Stress-strain plots for P2PIL with PEEK as resin.

It is evident from Table 10 and

Table 11 that the elastic modulus value corresponding to each configuration for the fiber and matrix are greater in the case of $\mathrm{X} / \mathrm{Z}$ direction loading. Hence, it is concluded that, the composite material is stronger in $\mathrm{Z}$ direction (major fiber alignment) when compared to $\mathrm{X}$ direction. Thus results are in agreement according to the physics of the configuration (Principle of composites i.e., material is stronger along major percent fiber volume and is weaker in other direction).

Table 10 Ply to Ply Interlocking without Warp - elastic modulus (GPa) Longitudinal

\begin{tabular}{ccc}
\hline Miber $\downarrow$ & PEEK & UHMWPE \\
\hline CF & & \\
Ti6Al4V & 81.42 & 60.93 \\
CoCr alloy & 48.12 & 35.10 \\
& 62.94 & 40.23 \\
\hline
\end{tabular}


Table 11 Ply to Ply Interlocking without Warp - elastic modulus (GPa) Transverse

\begin{tabular}{ccc}
\hline Matrix/resin $\rightarrow$ & PEEK & UHMWPE \\
\hline CF & & \\
Ti6Al4V & 18.34 & 11.56 \\
CoCr alloy & 4.25 & 16.08 \\
& 16.11 & 23.14 \\
\hline
\end{tabular}
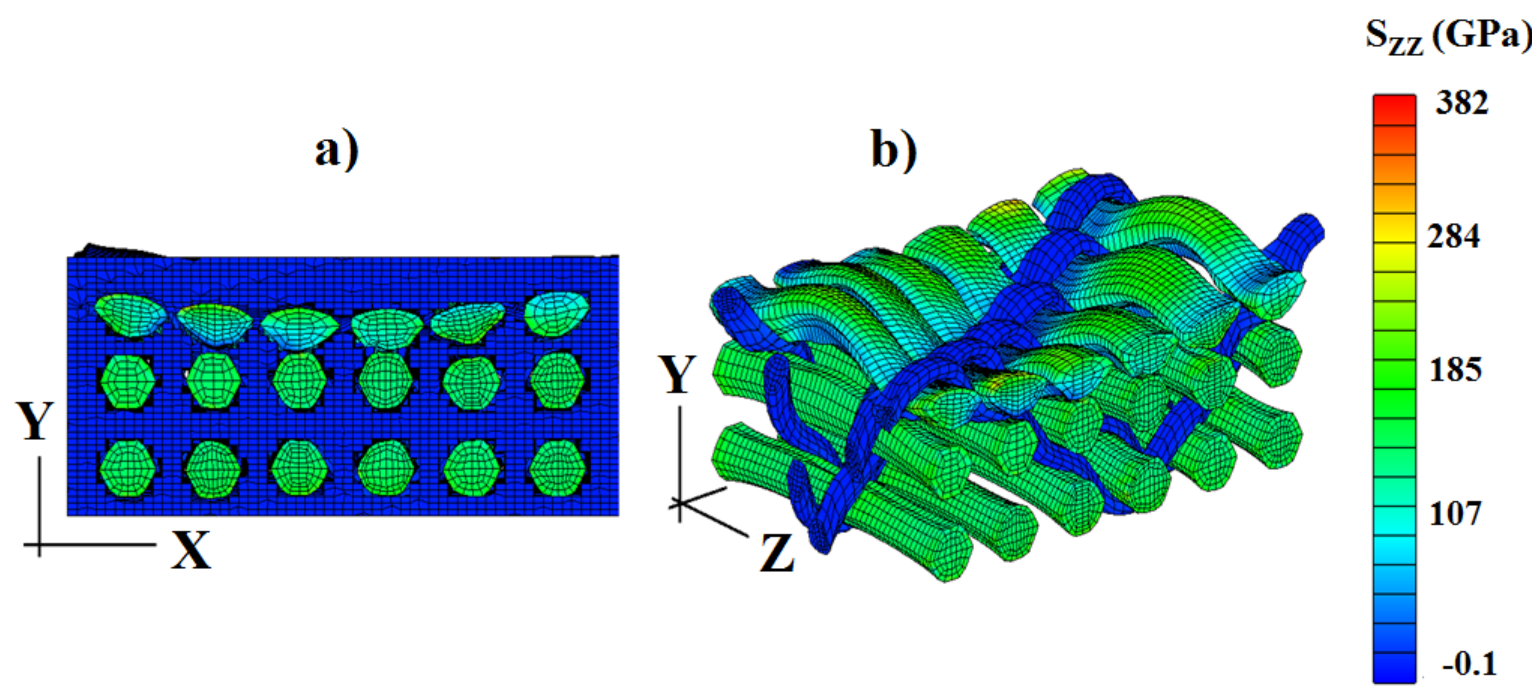

Figure 40 Stress Szz of P2PAng loaded in Z direction (a) Unit Composite (b) Fibers only

Stress distribution of P2PAng is shown in Figure 40 with loading in $\mathrm{Z}$ direction for both the unit cell and fiber only configurations. The fibers in the weft direction are more stressed in this load setting with an average stress value of $38.2 \mathrm{MPa}$. The simulation results provide principle stresses and principle strain matrices. The numerical values of the elastic modulus calculated from Stress strain plots in the longitudinal directions are summarized in Table 12. Similar analysis is performed for this architecture with loading 
in $\mathrm{X}$ direction. The inter-locking fibers are more stressed in this load setting with average stress value of 6.5 MPa. Elastic modulus in transverse direction are calculated from the analysis results and are summarized are in Table 13.

It is evident from Table 12 and Table 13 that the elastic modulus value corresponding to each configuration for the fiber and matrix are greater in the case of $\mathrm{X} / \mathrm{Z}$ direction loading. As expected the composite is stronger in $\mathrm{Z}$ direction due to more fiber volume percent aligned in this direction than $\mathrm{X}$ direction.

Table 12 Ply to Ply Angle Interlocking without Warp - elastic modulus (GPa) Longitudinal

\begin{tabular}{ccc}
\hline Matrix/resin & UHMWPE & PEEK \\
\hline CF & & \\
Ti6Al4V & 47.82 & 60.93 \\
CoCr alloy & 31.85 & 35.104 \\
& 58.95 & 62.94 \\
\hline
\end{tabular}

Table 13 Ply to Ply Angle Interlocking without Warp - elastic modulus (GPa) Transverse

\begin{tabular}{ccc}
\hline Matrix/resin & UHMWPE & PEEK \\
\hline CF & & \\
Ti6Al4V & 3.17 & 11.56 \\
CoCr alloy & 4.25 & 8.61 \\
\hline
\end{tabular}



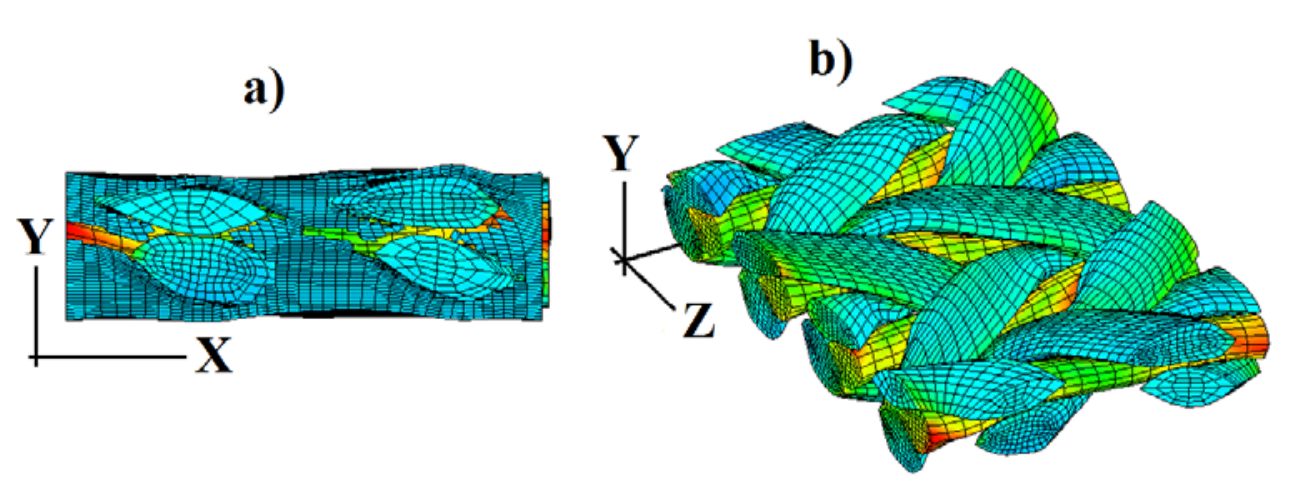

$\mathrm{S}_{\mathrm{xx}}(\mathrm{GPa})$

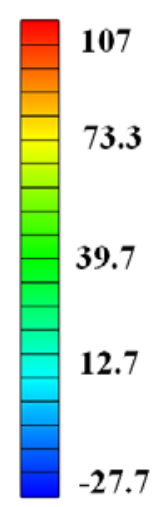

Figure 41 Stress Sxx of Traxial Braid loaded in X direction (a) Unit Composite (b) Fibers only

Stress distribution of Triaxial is shown in Figure 41 with loading in $\mathrm{X}$ direction for both the unit cell and fiber only configurations. The fibers in the warp direction are more stressed in this load setting with an average stress value of 10.7 MPa with one face fixed and other face loaded at $0.01 \mathrm{~mm}$. The simulation results provide principle stress and principle strain matrices. The numerical values of the Young's modulus calculated from Stress-strain plots in the longitudinal directions are summarized in Table 14. Similar analysis is performed for this architecture with loading in $\mathrm{Z}$ direction. The inter-weaved fibers are more stressed in this load setting with average stress value of 5.62 MPa with one face fixed and other face loaded at $.015 \mathrm{~mm}$. Elastic modulus in transverse direction are calculated from the analysis results and are summarized are in Table 15.

It is evident from Table 14 and Table 15 that the elastic modulus value corresponding to each configuration for the fiber and matrix are greater in the case of $\mathrm{X}$ direction loading. Hence, it is concluded that, the composite material is stronger in $\mathrm{X}$ direction 
(major fiber alignment) when compared to $\mathrm{Z}$ direction. The highest value of the elastic modulus (47.96 GPa) is for the composite with CoCr alloy and PEEK.

Table 14 Tri-axial - elastic modulus (GPa) Longitudinal

\begin{tabular}{ccc}
\hline & & \\
\hline Matrix/resin & UHMWPE & PEEK \\
\hline Ti6Al4V & 33.12 & 39.62 \\
CoCr alloy & 25.06 & 29.56 \\
& 42.65 & 47.96 \\
\hline
\end{tabular}

Table 15 Tri-axial - elastic modulus (GPa) Transverse

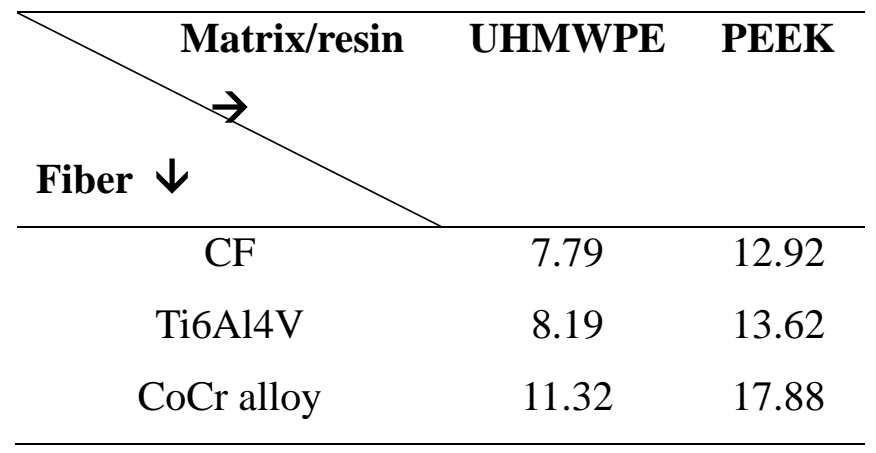

Analysis of four different architectures with various combinations of fiber and resin were considered, which resulted in 24 different sets of values for the Young's Modulus. The results were compared to the desired elastic moduli criterion (for reducing the stress shielding factor). These are to be between 40 and 95 GPa in longitudinal direction and between 15 and $30 \mathrm{GPa}$ in transverse direction. One set of results for all architectures and fiber combinations with PEEK as resin is shown in Figure 42 for comparison. This figure shows Young's Modulus in longitudinal and transverse directions. From the bar graph, the elastic modulus of P2PIL and P2PAng architectures are between the desired ranges of 
40 and 95 GPa in longitudinal direction. Similarly Ortho and P2PIL architectures satisfy the desired criteria of 15 to $30 \mathrm{GPa}$ in transverse direction. Overall, P2PIL is good in both longitudinal and transverse directions.
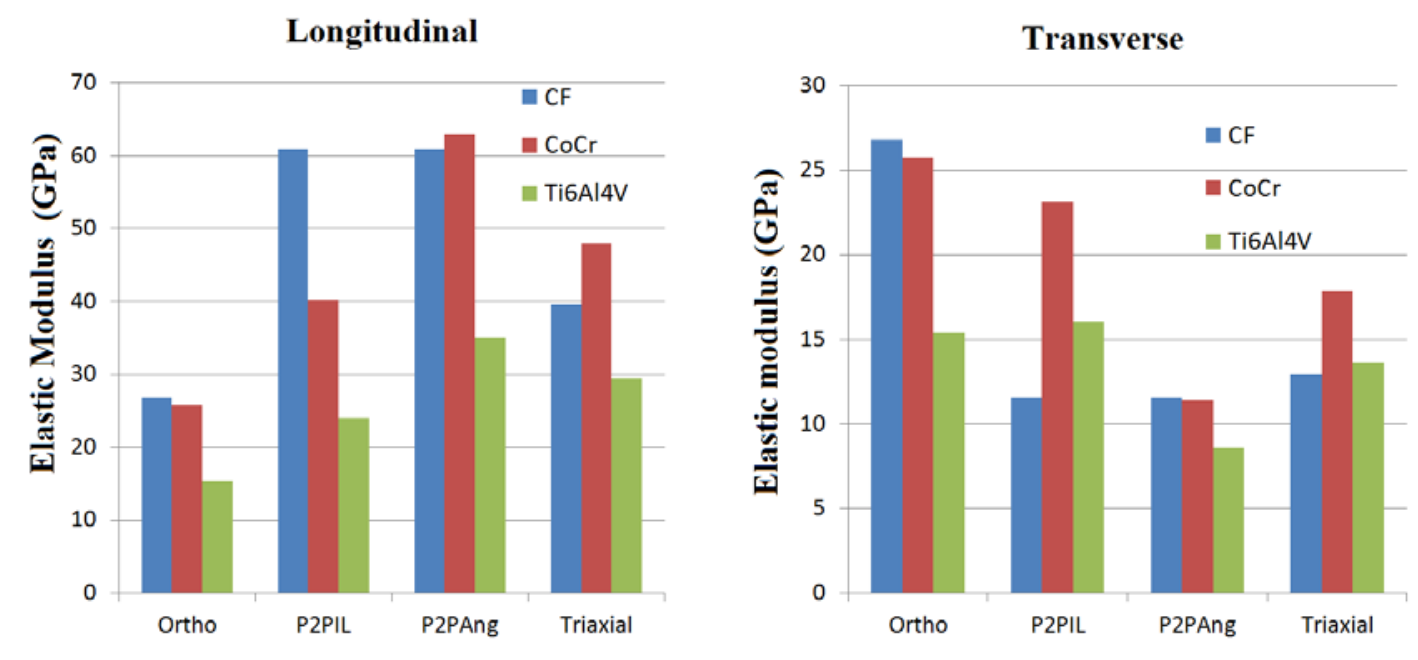

Figure 42 Elastic Modulus for different architectures and fibers with PEEK as resin

Among the four different architectures, P2PIL and P2PAng with Carbon Fiber and UHMWPE show promising strength values of approximately 81.4 GPa in longitudinal direction and approximately 18.3 GPa in transverse direction. Using CoCr alloy as fiber material also resulted in desired strength values (62.94 GPa). Hence the structural response of two different architectures are suitable for the use in hip implants.

\subsection{Statistical analysis - ANOVA}

An analysis of variance (ANOVA) test was carried out with results from microanalysis, using SPSS21 and Minitab, to demonstrate the significance (or insignificance) of various factors on the bulk elastic modulus. A multi-factorial model is established, which included basic factors (a) different architectures (b) different fibers (c) different 
matrix (d) different orientations. The linear model with interactions is based on the following equations,

$$
y=a_{0}+\sum_{i=1}^{4} a_{i} x_{i}+\sum_{j=1}^{4} \sum_{i=1}^{4} a_{i j} x_{i} x_{j}+\sum_{k=1}^{4} \sum_{j=1}^{4} \sum_{i=1}^{4} a_{i j k} x_{i} x_{j} x_{k}+a_{1234} x_{1} x_{2} x_{3} x_{4}=\boldsymbol{A} . \boldsymbol{x}
$$

The variables $x_{1}, x_{2}, x_{3}$ and $x_{4}$ represent four independent variable with different levels.

Table 16 Independent factors considered for ANOVA

\begin{tabular}{cc}
\hline Variable Name & Levels \\
\hline Architecture & 4 \\
Fibers & 3 \\
Resin & 2 \\
Orientation & 2 \\
\hline
\end{tabular}

The independent variable architecture included four levels- 1) 3D Orthogonal Woven Architecture (3DOW) 2) Ply 2 Ply Interlock without Warp (P2PILwoW) 3) Ply to Ply Angle Interlock without Warp (P2PAngILwoW) 4) Triaxial Braid. The independent variable fiber included three levels - 1) Carbon Fiber 2) Ti6Al4V 3) CoCr Alloy. The independent variable matrix included two levels - 1) UHMWPE 2) PEEK. The independent variable orientation are separated to two levels - 1) Longitudinal 2) Transverse.

With all the possible combinations, a set of $4 \times 3 \times 2=24$ modeling effects $A_{i}$ can be established separately for each set of results. The test hypothesis can be expressed as

$$
\begin{gathered}
H_{0}: A_{1}=\cdots=A_{24}=0 \\
H_{a}: \text { Atleast one } A_{i} \neq 0
\end{gathered}
$$


In other words, the null hypothesis $H_{0}$ states that there is no significant statistical difference between young's moduli found from architecture, fiber, resin /matrix and direction/ orientation while the alternate hypothesis $H_{a}$ states that there is atleast one factor affecting the elastic modulus of the woven composite. The results of ANOVA from Minitab are given below:

\section{General Linear Model: Elastic Modulus versus Architecture, Fiber, ...}

\begin{tabular}{|c|c|c|c|c|c|c|c|}
\hline Factor & \multicolumn{2}{|c|}{ Type Leve } & Value & & & & \\
\hline Architecture & \multicolumn{2}{|c|}{ fixed } & 1,2 , & 3,4 & & & \\
\hline Orientation & \multicolumn{2}{|c|}{ fixed } & $\odot, 90$ & & & & \\
\hline Fiber & \multicolumn{2}{|c|}{ fixed } & 1,2 , & 3 & & & \\
\hline Matrix & \multicolumn{2}{|c|}{ fixed } & 1,2 & & & & \\
\hline Analysis of $\mathrm{V}$ & \multicolumn{2}{|c|}{ Variance } & lastic $N$ & odulus, & using $A$ & djusted & SS for Tests \\
\hline Source & DF & Seq SS & Adj SS & Adj MS & $\mathrm{F}$ & $\mathrm{P}$ & \\
\hline Architecture & 3 & 223.4 & 1764.5 & 588.2 & 5.14 & 0.005 & \\
\hline Orientation & 1 & 9679.7 & 9326.2 & 9326.2 & 81.56 & 0.000 & \\
\hline Fiber & 2 & 1278.3 & 1306.1 & 653.1 & 5.71 & 0.007 & \\
\hline Matrix & 1 & 32.4 & 32.4 & 32.4 & 0.28 & 0.598 & \\
\hline Error & 33 & 3773.6 & 3773.6 & 114.4 & & & \\
\hline Total & 40 & 14987.5 & & & & & \\
\hline
\end{tabular}

The multivariate tests indicate a significant main effect of architecture with $F(3,40)$ $=5.14, \mathrm{p}$ value $=0.005$, a significant main effect of fibers with $F(2,40)=5.71, \mathrm{p}$ value $=0.007$, a non-significant main effect of resin/matrix with $F(1,40)=0.28$, p value $=0.598$, a significant main effect of orientation with $F(1,40)=81.56$, p value $<0.001$. The ANOVA found no significant interaction effect, so a follow up pairwise comparisons of main effects are performed. 


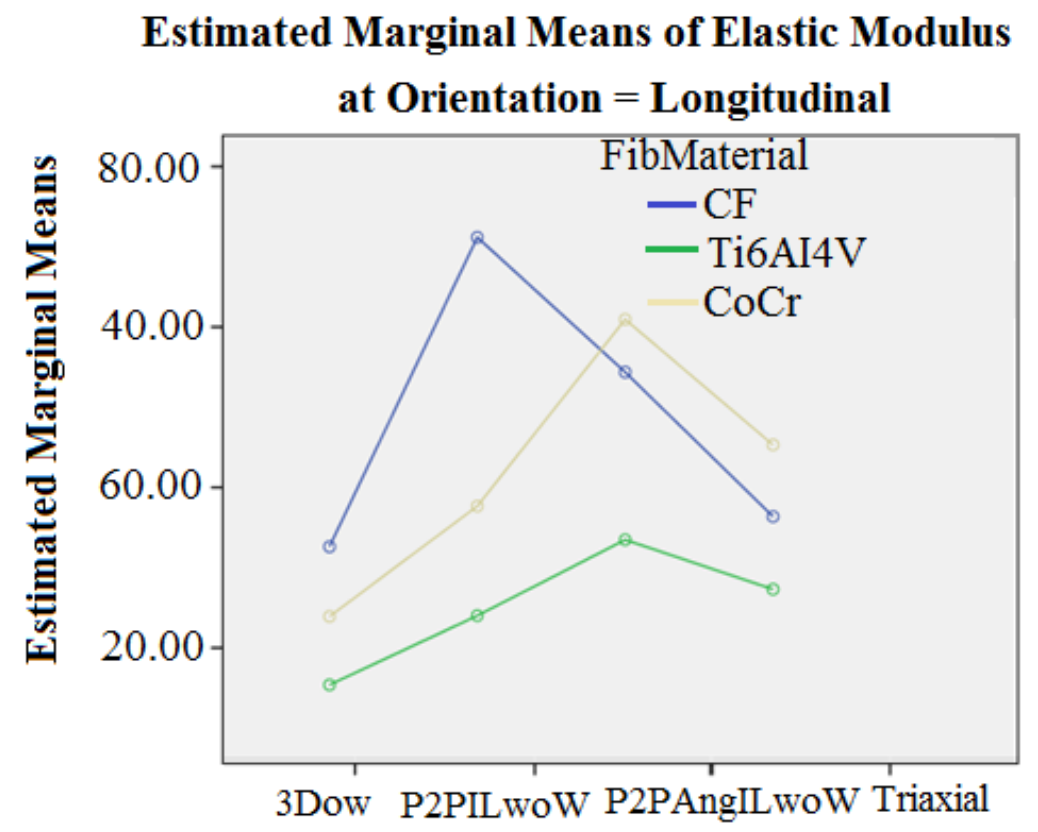

\section{Architecture}

Figure 43 Estimated marginal mean elastic modulus (GPa) in longitudinal direction

\section{Estimated Marginal Means of Elastic Modulus}

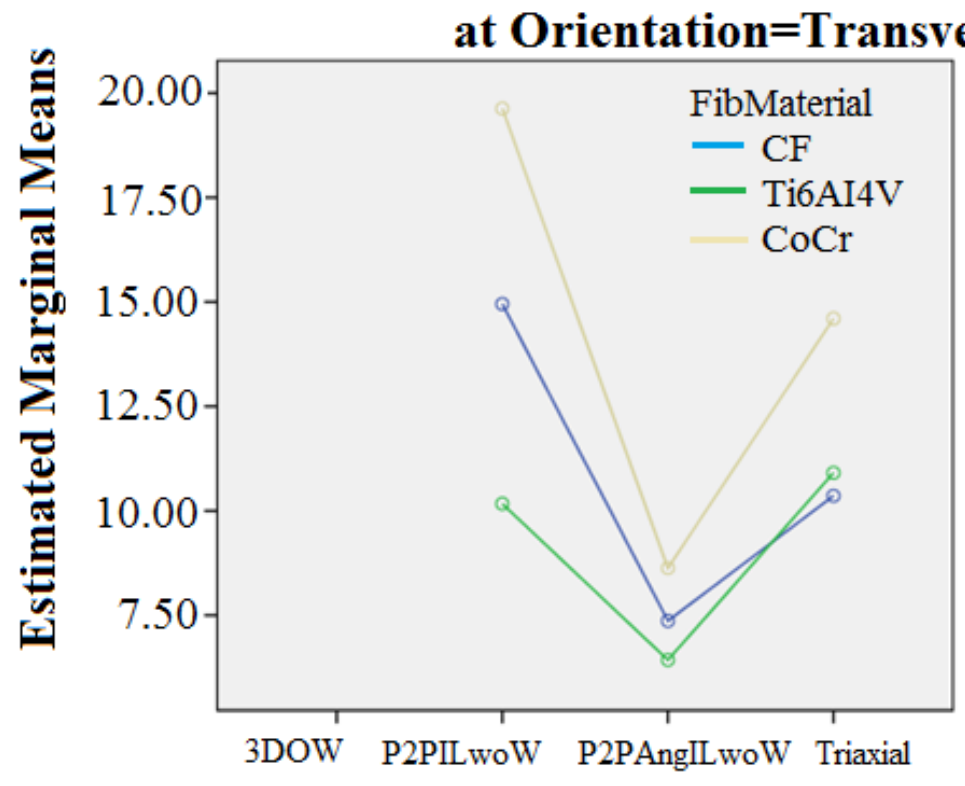

\section{Architecture}

Figure 44 Estimated marginal mean elastic modulus (GPa) in transverse direction 


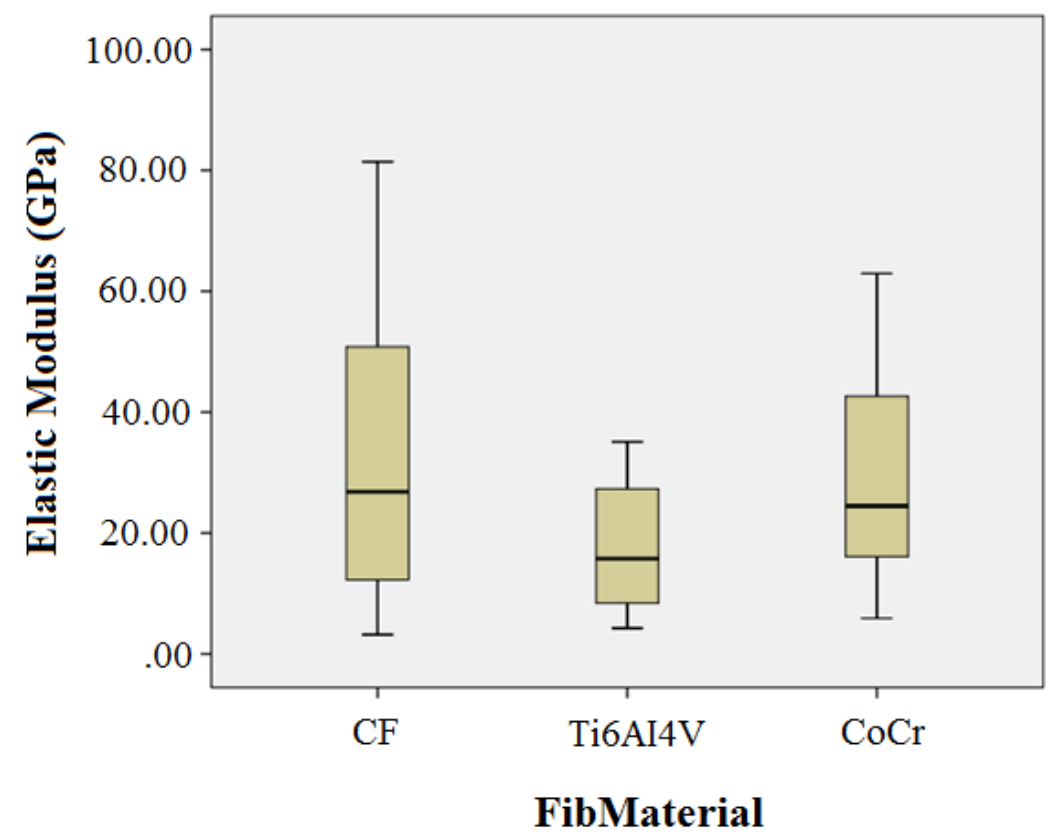

Figure 45 Box plot showing mean and variance of elastic modulus w.r.t fibers

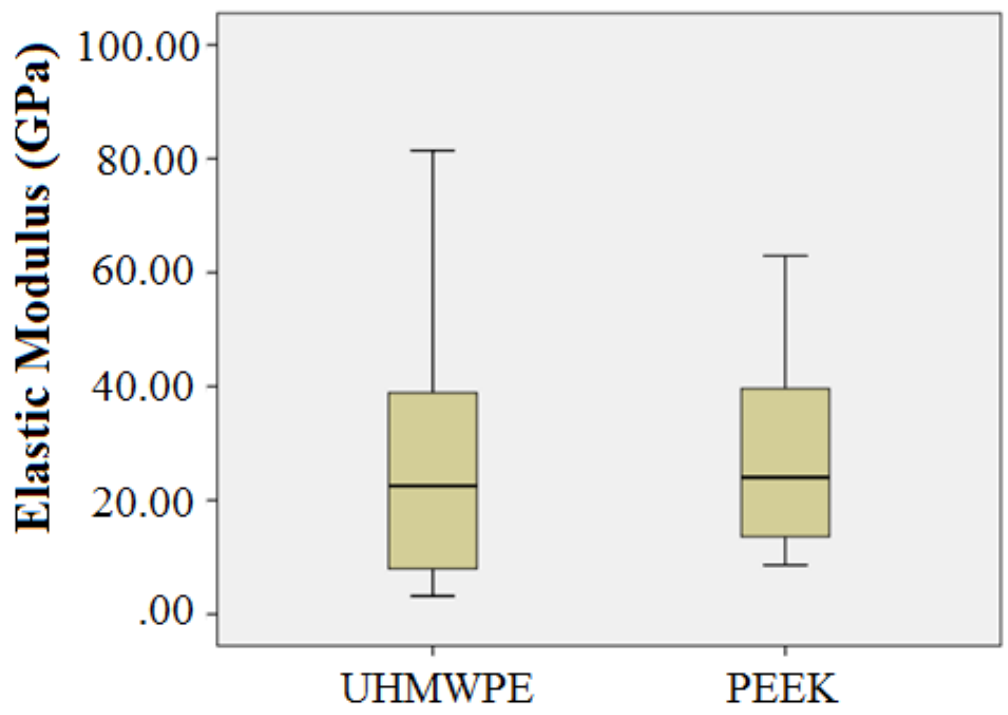

MatrixMaterial

Figure 46 Box plot showing mean and variance of elastic modulus w.r.t matrix 


\subsubsection{Test for Normality}

Residual plots (Figure 47) are drawn to test for normality assusmption indicating that the developed architectures and fiber sample sets are considered to be unbiased and statistically correct. The normal PP plots show that the data follows normality. Also the fitted residual plots show no obvious repeating pattern. These two indicate data collected from micro-analysis follows normal distribution.

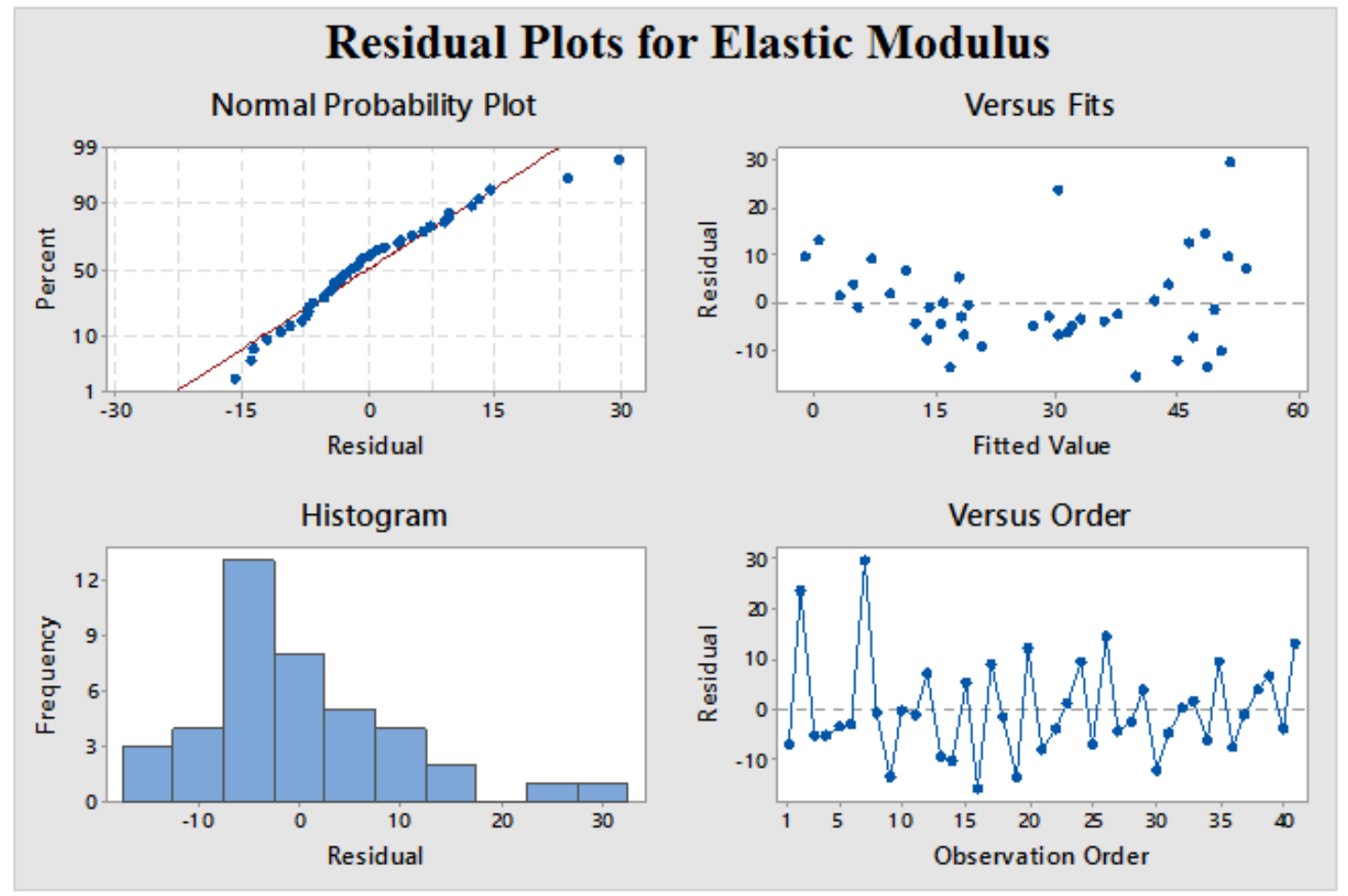

Figure 47 Residual plots for elastic modulus

\subsection{FEA results of hip implants}

This section provides the results obtained from the static analysis of the hip implant. After completing static analysis in Abaqus, the results are reviewed in the visualization of Abaqus and HyperView. Results from mesh refinement are plotted. The main significant results for all the four models are recorded and reported in this section. Results for von 
Mises stress, deflection and maximum principal stress are also presented for all the four different models.

\subsubsection{Results for mesh refinement}

The displacement magnitude (in mm) plots for implant model 1 are shown in Figure 48. This figure shows, both value and location of the maximum and minimum displacements in the stem, for target element size of 1, 2, 3 and 5. In Figure 49 the von Mises stress for these different element sizes are also shown. The maximum and minimum stress values along with their locations are also indicated in this figure.

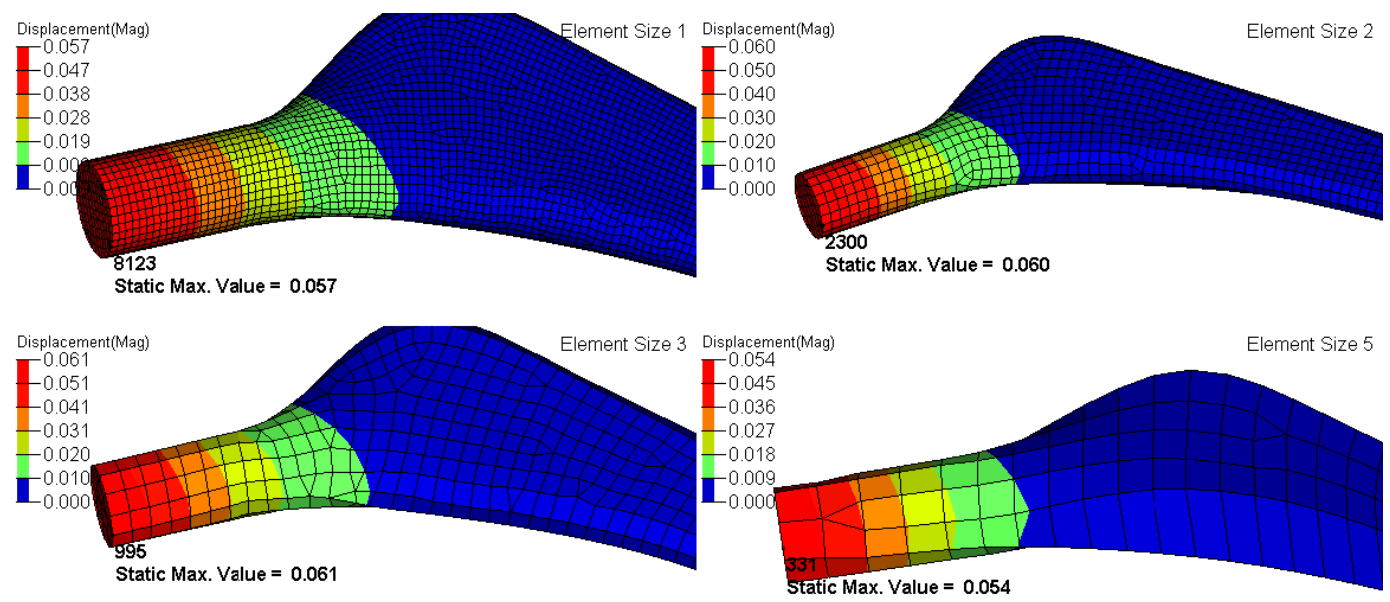

Figure 48 Implant 1 -Displacement (in mm) using different mesh sizes 


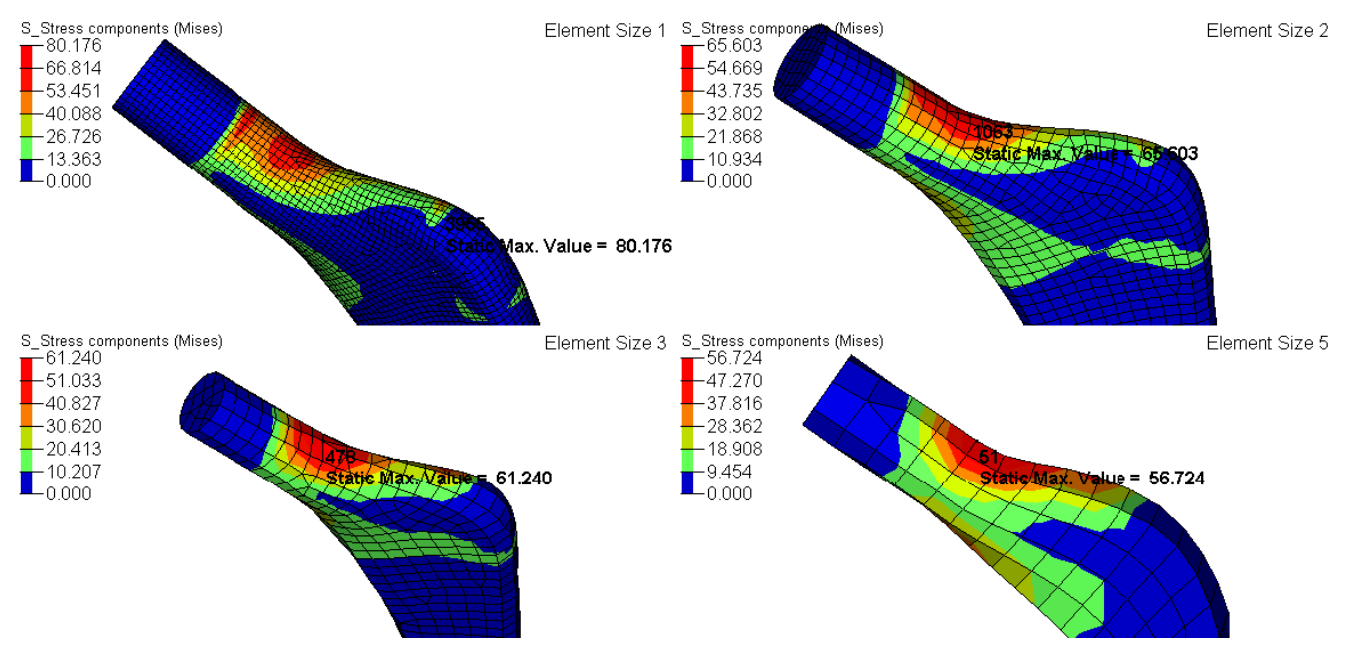

Figure 49 Implant 1 - von Mises Stress (in MPa) using different mesh sizes

Two paths are chosen on the stem, Path1 on the surface and Path2 near center line as shown in Figure 50 and the corresponding displacements are plotted against true distance travelled on the stem, The displacements obtained on path1, with different element sizes are plotted in Figure 51 and displacements obtained on path2 in Figure 52, together to test for convergence of FEA i.e., to determine appropriate mesh size for the model. From the meshed model Figure 32 Figure 33, it was evident that target mesh size of 5 and 4 were unable to capture all the geometry of the implant model. Target element sizes of 3, 2 and 1 appear to be reasonable. All the five different element size models were able to capture the location of the maximum and minimum at the same location on the stem. However, the displacement plots shows that the change in percentage is beyond $10 \%$ between element sizes 5, 4 and 3. Whereas the overall percentage change is below $10 \%$ between element sizes 3, 2 and 1. The use of very fine mesh will exponentially increase the FEA computational time. Since target element sizes 3, 2 and 1 show convergence, a target element size of 2 is chosen for the rest of the models. Due to different element sizes, the 
maximum von Mises stress reported is different due to changes in elemental forces and area. But, the location of maximum stress was near the same spot in all the models. The maximum von Mises stress is $56.72 \mathrm{MPa}$ on the stem of implant 1 for a target element size of 5, 61.24 MPa for a target element size of 3, 65.60 MPa for a target element size of 2 and 80.17 MPa for a target element size of 1 . Similarly the maximum displacement is $0.054 \mathrm{~mm}$ for a target element size of 5, $0.061 \mathrm{~mm}$ for a target element size of 3, 0.060 $\mathrm{mm}$ for a target element size of 2 and $0.057 \mathrm{~mm}$ for a target element size of 1 .

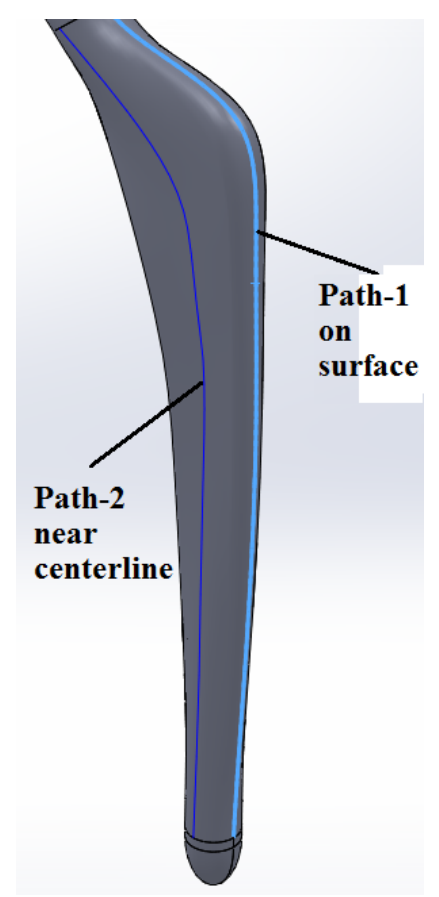

Figure 50 Path1 on surface of stem for displacement and stress plots 


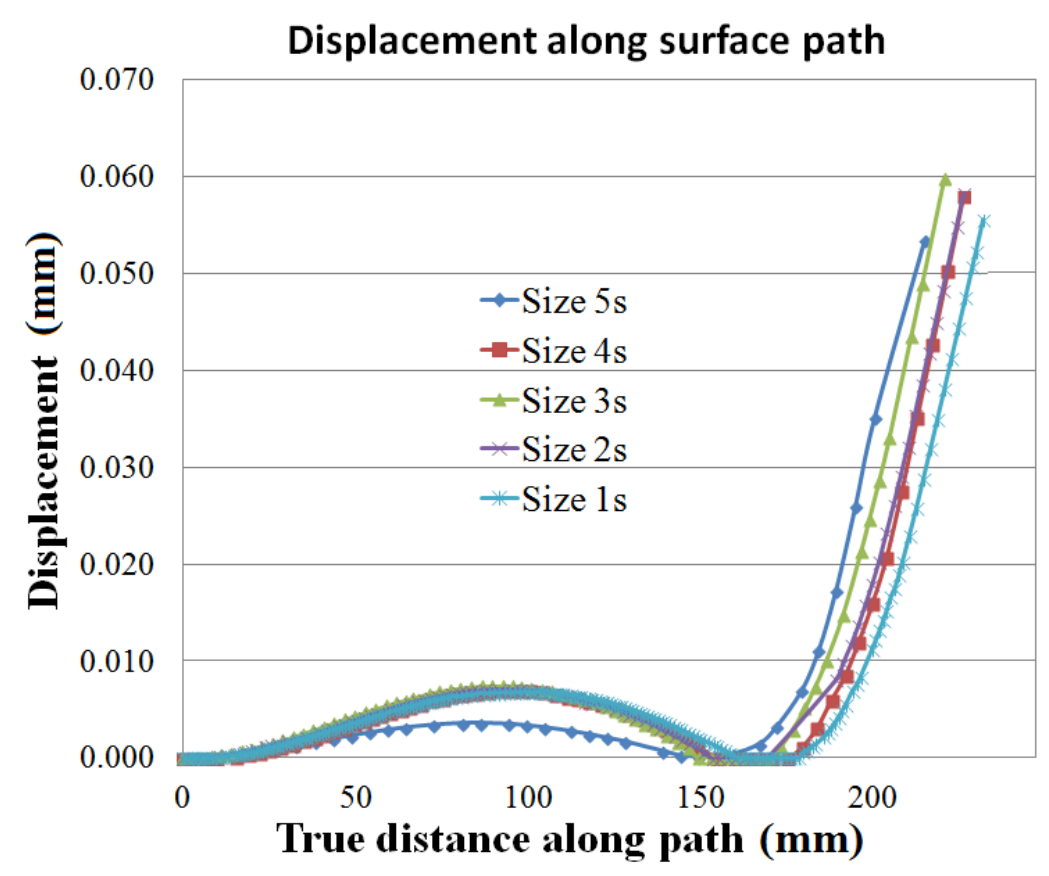

Figure 51 Implant1- displacement along the surface path with different mesh sizes

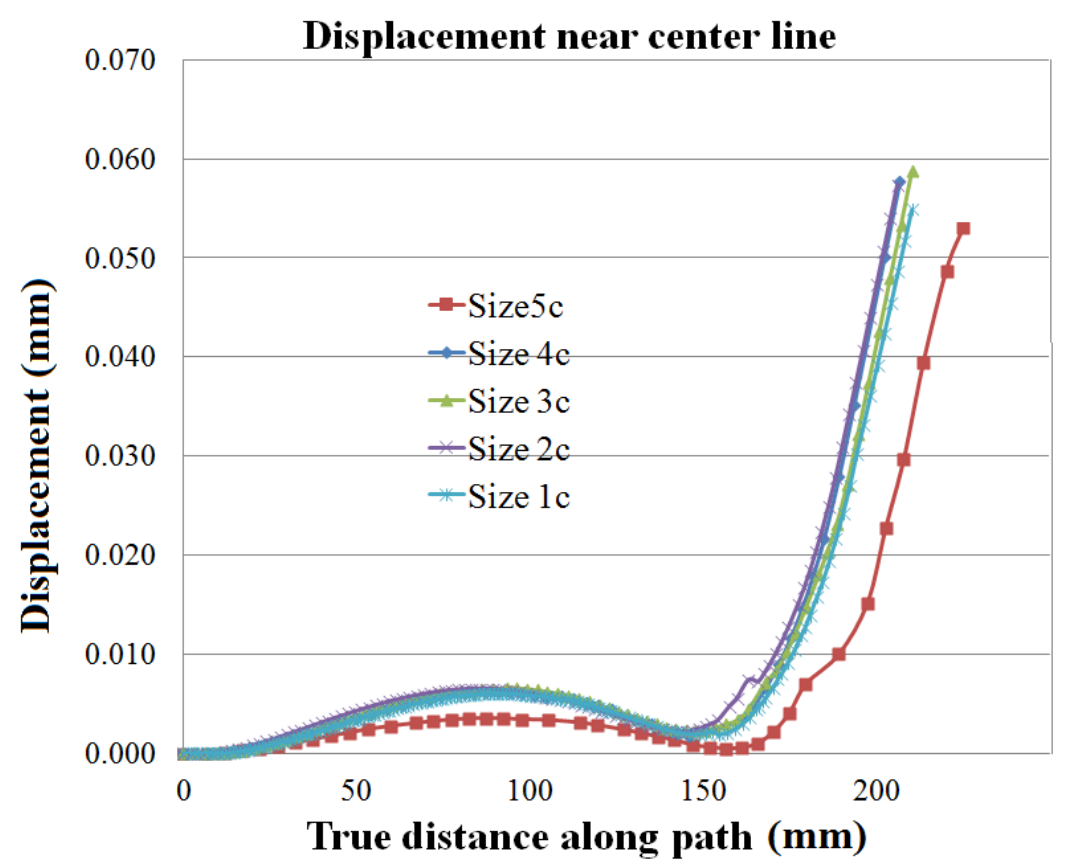

Figure 52 Implant1- displacement near centerline with different mesh sizes 
After mesh refinement and concluding a target element size of 2 is more accurate, FEM analysis is performed on the rest of the three models.

Similar displacement and stress plots are drawn, the magnitude and location of maximum and minimum values are recorded. The maximum displacement of stem in Implant 2 is $0.05 \mathrm{~mm}$, the maximum von Mises stress is $60.32 \mathrm{MPa}$ and the maximum principal stress is $62.05 \mathrm{MPa}$. The maximum displacement of stem in Implant 3 is found to be $0.064 \mathrm{~mm}$, the maximum von Mises stress is $69.57 \mathrm{MPa}$ and the maximum principal stress is 75.63 MPa. The maximum displacement of stem in Implant 4 is found to be $0.051 \mathrm{~mm}$, the maximum von Mises stress is $65.34 \mathrm{MPa}$ and the maximum principal stress is 67.39 MPa.

\subsubsection{Comparison of Implants models}

Implant 1 and 3 are identical in size and other dimensions. However, implant 1 is made of regular Ti alloy and functionally graded material is considered in implant 3. Displacement plots are drawn along the two path and are compared for these two models as shown in Figure 53. It is observed that there is approximately $10 \%$ increase in the deflection due to the use of functionally graded material. Plots of von Mises stress are also drawn to compare the stress distribution along the two paths shown in Figure 54. From the stress plots, we see that the stress is distributed over larger area of the stem rather than concentrating near the neck region as seen in implant 1 . We also found that von Mises stresses are more in implant 3 than implant 1 


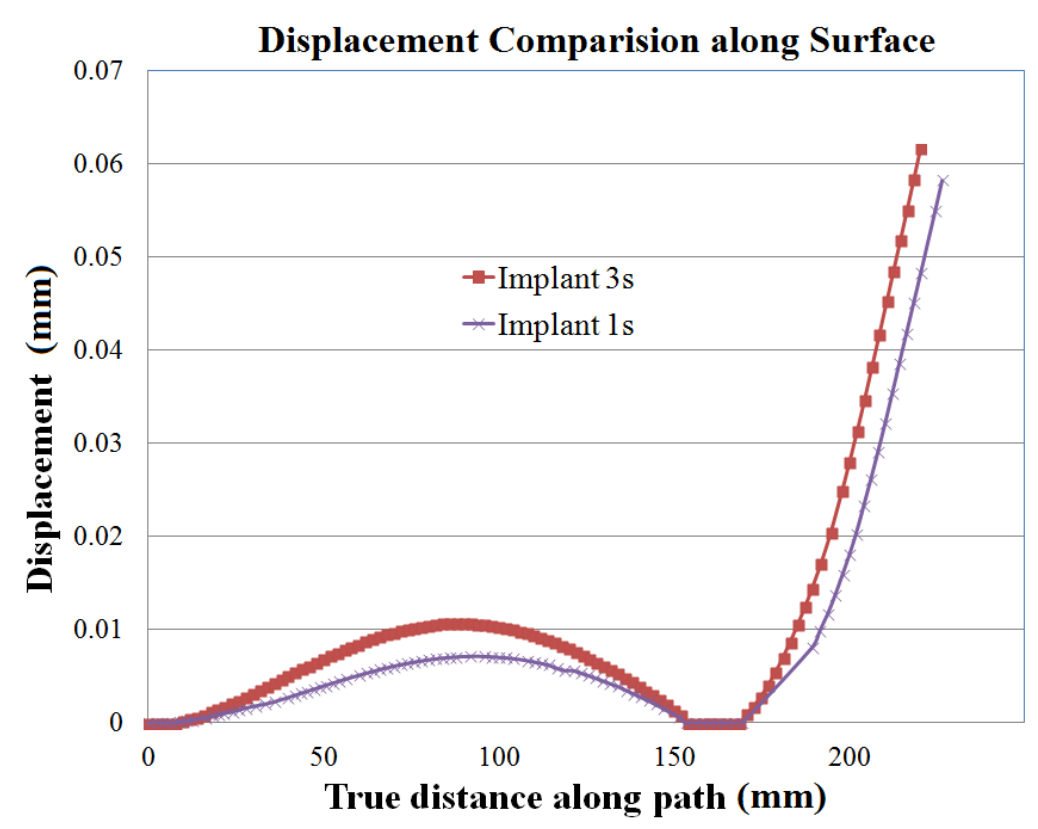

Figure 53 Displacement plot on Implant 1 and 3 on surface

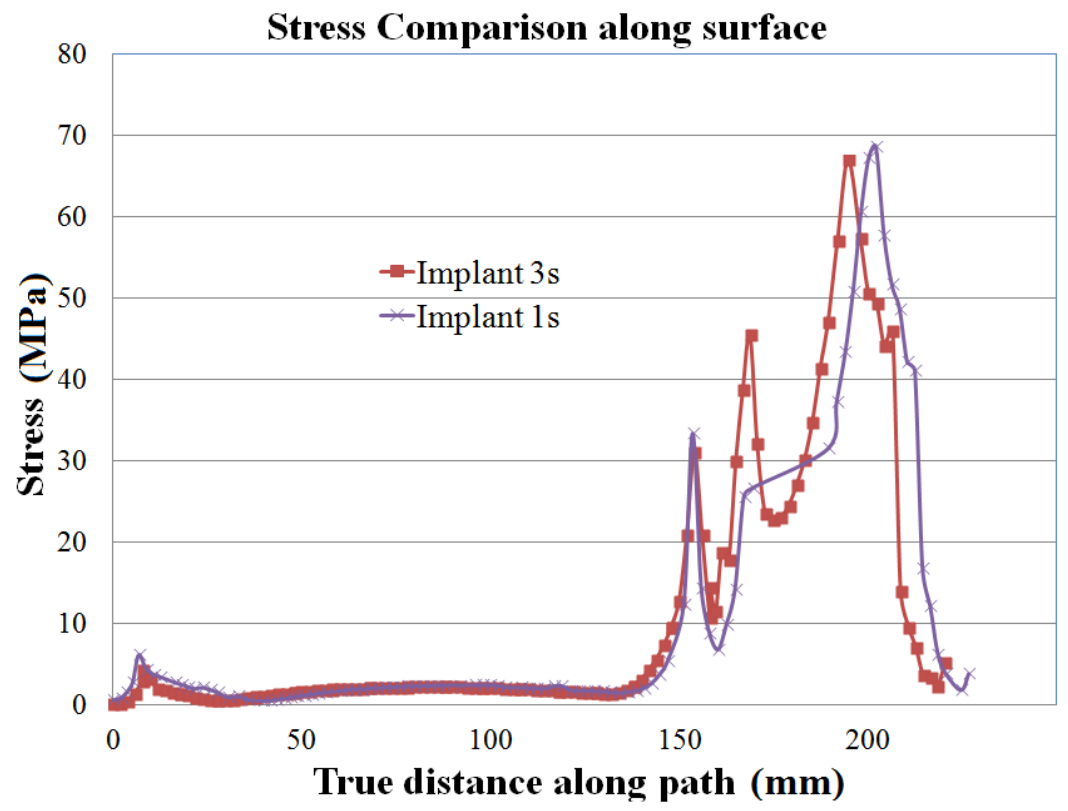

Figure 54 Stress plot on Implant 1 and 3 on surface

Similarly, implant 2 and 4 are identical in size and other dimensions. However, implant 2 is made of regular Ti alloy and functionally graded material is considered for implant 4 . 


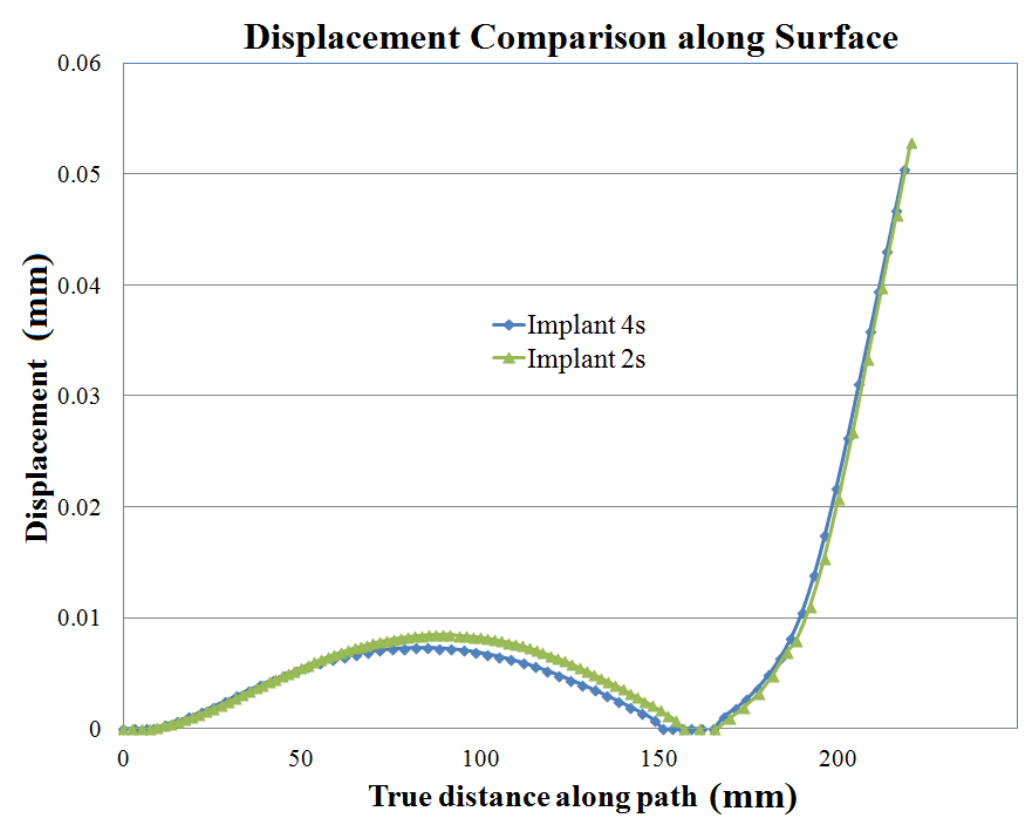

Figure 55 Displacement plot on Implant 2 and 4 on surface

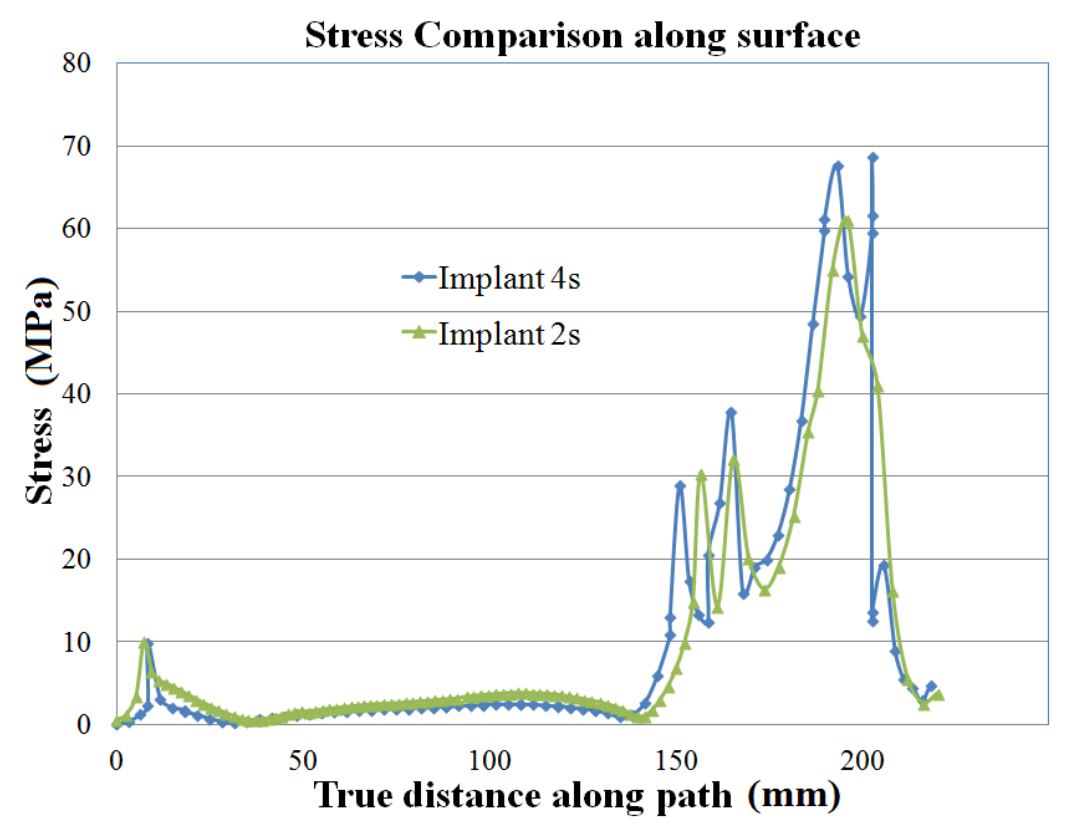

Figure 56 Stress plot on Implant 2 and 4 on surface 
Displacement plots are drawn along the two path and are compared for these two models as shown in Figure 55. It is observed that there is approximately 5\% increase in the deflection due to the use of functionally graded material. Plots of von Mises stress are also drawn to compare the stress distribution along the two paths shown in Figure 56. From the stress plots, we noticed similar effect on stress distribution as before. 


\section{CHAPTER 7-Conclusions and future work}

In the present work, micro mechanical models of 3DWC architectures are developed to incorporate them in bio-medical applications, specifically in the hip implants. Four different woven architecture models are developed based on physics based approach and bulk material properties of each architecture are determined using b-spline analysis method. Out of four different architectures considered, two of them showed promising results, which met the targeted elastic modulus. Two of the three different fiber materials chosen are considered significant, however any matrix can be chosen for the design.

The results from micro-mechanics are incorporated to resemble a functionally graded material and these material properties are considered to design the stem of hip implant. The comparison of von Mises stresses with base model showed an overall stress increase of approximately $10 \%$. We observed increase in stresses near the distal end of the implant, which might benefit in reducing the stress shielding effect [79]. We also noticed increase in the displacement of the stem between the distal end and the neck region of the implant. The desirable limit level of the micro-motions for bone ingrowth is approximately $20 \mu \mathrm{m}$ to $50 \mu \mathrm{m}$, whereas the micro-motion above 50 and $150 \mu \mathrm{m}$ results in disruption of Osseo-integration and the formation of a fibrous tissue layer that weakens the interface and thus causes loosening [79]. However, no precise value is known for the interface stress and micro-motions [80]. This increased displacement is within the acceptable range of motion which indicates possible strengthening of the femur bone. Even though we did not incorporate variation of densities in our model, in reality, the composite material is less dense which makes the hip implant much lighter than the one currently available. 
In future, this micro-mechanics models can be used to incorporate advanced fiber and resin materials. Further, failure analysis like Hasin’s failure criterion can be implemented for these orthotropic architectures. In the present work, interface between fibers and matrix is assumed to be perfectly bonded, thus applying tie constraints to these interface nodes. In future, instead of assuming perfect interface bonding, penalty method or cohesive interface bonding or Virtual Crack Closure Technique (VCCT) approaches available in Abaqus, can be applied to the interfaces. Micromechanical strength modeling of textile composites using cohesive interface were performed by other researchers and this can be further expanded to this micro-mechanical analysis [81] [82].

More woven architectures twill woven, satin woven, ply to ply interlocking with warp, ply to ply angle interlocking with warp or even tubular biaxial and tubular triaxial can be considered for design space instead of current architectures specified in 4.3. Or the chosen architectures in 4.3, can be further optimized by varying the diameter, pitch, interlacing fibers and other parameters. For instance, at the beginning, we thought triaxial architecture would meet the desired requirements. An added advantage of this architecture is the interlacing braided fibers are at an angle which provide increased strength transverse directions compared to ply to ply and ply to ply angle interlock architectures. The strength needed in longitudinal direction was close to the requirement but did not pass the threshold values. So, further making changes in diameter, angle and diameter of braids, this architecture might pass the criteria.

In macro-analysis, for functionally graded material the variation of elastic modulus is controlled using power law equation (\#17) with $\mathrm{n}$ value of 0.5 . This can be further 
expanded to use different $n$ values ( 0 to 1 ) shown in Figure 57 which will control the nature of material variation.

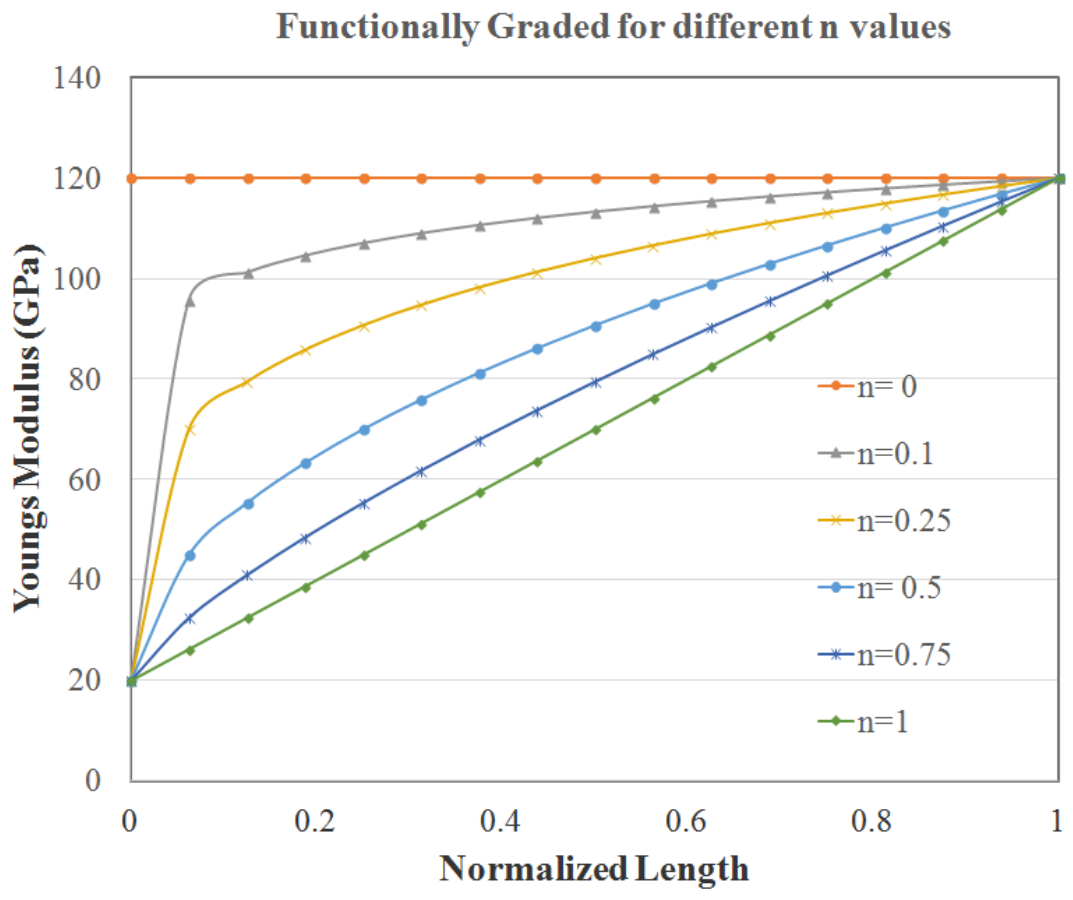

Figure 57 Functionally graded material properties for different n values

In some failure cases, surgeons observed breaking at the neck region of the stem, which is proven to be high stress region here and by several other researchers. In our study, we considered elastic modulus at the neck to reach 115 GPa. The study can further be expanded to target the strength at neck region to be near $200 \mathrm{GPa}$. In the present study, density of functionally graded composite is not varied and assumed to be a constant factor. This study can be further expanded incorporating changes in density of the functionally graded material. Also instead of discretizing functionally graded material to piece wise for FEA, a user defined sub-routine can be used to define the variation of 
functionally graded materials, which will further homogenize the stress distribution and displacements.

The present study is limited to static loading on the hip implant, which represents loading during normal walking. This load is one of the several loading cases within the gait cycle. In future, the study can be expanded to include all loading cases of the gait cycle mentioned in 2.3.1, different activities like running, jumping etc., and perform dynamic analysis.

Further studies include, determining the biocompatibility of these woven composites using different combinations of fiber and matrix in vivo and in vitro. The cell growth between the composite implant and femur bone need to be investigated. Due to the use of composites, drug-eluting implants (similar to stents) can be evaluated in the future to avoid foreign object rejection and other reaction at the surgical site needs to be evaluated. The composite implant can also be made porous to enhance bone growth into the implant.

This study can be further expanded to implement in other applications, such as knee implants, prosthesis in arms, implantable devices in spinal cord, jaws or areas where bone repair or growth is needed. Woven composites can be considered outside biomedical field where functionally graded structures are needed such as aerospace, structural and automobile industries. 
Bibliography

[1] S. Ramakrishna, J. Nayer, E. Wintermantel and K. W. Leong, "Biomedical applications of polymer composite materials: a review," Composite Science Technology;61(9), pp. 1189-1224, 2001.

[2] H. Bougherara, R. Zdero, A. Dubov, S. Shah, S. Khurshid and E. H. Schemitsch, "A preliminary biomechanical study of a novel carbon fibre hip implant versus standard metallic hip implants.," Medical Engineering \& Physics, vol. 33, no. 1, pp. 121-8, 2011.

[3] C. A. Engh, J. D. Bobyn and A. H. Glassman, "Porous-coated hip replacement - The factors governing bone ingrowth, stress shielding, and clinical results," J. Bone Jt. S Urg., 69B, pp. 45-55, 1987.

[4] D. M. Dall, I. D. Learmonth, M. I. Solomon, A. W. Miles and J. M. Davenport, "Fracture and loosening of Charnley femoral stems," J. Bone Jt. Surg, 75B, pp. 259-265, 1993.

[5] H. B. Skinner and F. J. Curlin, "Decreased pain with lower flexural rigidity of uncemented femoral prostheses," Orthopedics, 13, pp. 1223-1228, 1990.

[6] C. Kaddick, S. Stur and E. Hipp, "Mechanical simulation of composite hip stems," Med. Eng. Phys. Vol. 19, No.5,, pp. 431-439, 1997.

[7] H. Valdemar, "Total Joints Information -Bananarepublican Stress Shielding," [Online]. Available: http://www.bananarepublican.info/Stress_shielding.htm. [Accessed 10 October 2015].

[8] B. Chen, E. J. Lang and T.-W. Chou, "Experimental and theoretical studies of fabric compaction behavior in resin transfer molding," Materials Science and Engineering, vol. 317, no. 1-2, p. 188-196, 2001.

[9] Y. .. Miao, E. .. Zhou, Y. .. Wang and B. Cheeseman, "Mechanics of textile composites: Micro geometry," Journal of Composites Science and Technology, 2008.

[10] P. Potluri and T. Sagar, "Compaction modelling of textile performs for composite structures," Elsevier - Composite Structures 86, pp. 177-185, 2008.

[11] S. D. Ranajay Ghosh, "Z-fiber influence on high speed penetration of 3D orthogonal," Elsevier - Mechanics of Materials 68 , p. 147-163, 2014.

[12] S. Green, M. Matveev, A. Long, D. Ivanov and S. Hallett, "Mechanical modelling of 3D woven composites considering realistic unit cell geometry," Elsevier -Composite Structures 118, p. 284-293, 2014. 
[13] B. Cox, H. Bale, M. Blacklock, B. C. Do, R. Rinaldi, R. Ritchie, Q. Yang, F. Zok and D. Marshall, "A Pipeline Approach to Developing Virtual Tests for Composite Materials," in 13th International Conference on Fracture, June 16-21, Beijing, China, 2013.

[14] F. F. .. Al-Jassir, H. Fouad and O. Y. Alothman, "In vitro assessment of Function Graded (FG) artificial Hip joint stem in terms of bone/cement stresses: 3D Finite Element (FE) study," Al-Jassir et al. BioMedical Engineering OnLine , 12:5, pp. 1-17, 2013.

[15] Z. Hongyu, "The Influence of Stem Design and Fixation Methods on the Lifetime of Total Hip Replacement," thesis, 2009.

[16] N. P. Palastanga, D. a. Field and R. Soames, "Anatomy and Human Movement: Structure and Function," Butterworth Heinemann, 2006.

[17] R. Martin, D. a. Burr and N. Sharkey, "Skeletal tissue mechanics," Springer Verlag, New York, 1998.

[18] L. a. Junqueira and J. Carneiro, "Basic Histology: Text \& Atlas," McGrawHill, 2005.

[19] B. Encyclopedia, " The cancellous bone in the interior of long bones," 19 April 2012. [Online].

[20] "Science Photo Library, Cancellous bone trabeculae," 15 July 2012. [Online]. Available: http://www.britannica.com/science/cancellous-bone. [Accessed 20 Aug 2015].

[21] H. Gray, "Neuroanatomy," Churchill Livingstone,2nd edition, 2000.

[22] F. H. Netter, J. a. Hansen and D. Lambert, "Netter's clinical anatomy," Icon Learning Systems, 2008.

[23] Antranik, "Cartilage and Bones on Anrtranik," 27 September 2011. [Online]. Available: http://antranik.org/wp-content/uploads/2011/09/bone-design-andstress-compression-decompression-body-weight.png. [Accessed 12 October 2015].

[24] J. P. Paul, "Forces transmitted by joints in the human body," Proceedings of Institution of Mechanical Engineers, pp. 181, 8-15, 1966.

[25] C. Vaughan, B. a. Davis and J. O'Connor, "Dynamics of Human Gait," Kiboho, Cape Town, South Africa, 2nd edition, 1999.

[26] M. Whittle, "An introduction. Butterworth-Heinemann Medical," Gait analysis,4th edition, 2007. 
[27] A. Charlish, "The Complete Arthritis Handbook," Carnell Ltd, London, 1994.

[28] J. J. Callaghan, A. G. Rosenberg and H. E. Rubash, The Adult Hip, Lippincott Williams \& Wilkins, 2007.

[29] M. Corr, "Rheumatoid arthritis. Introduction," Springer Semin Immunopatholgy,, vol. 25, pp. 1-2., 2003.

[30] W. C. Shiel Jr., "Rheumatoid arthritis article in medicinenet," 15 June 2015. [Online]. Available: http://www.medicinenet.com/rheumatoid_arthritis/article.htm. [Accessed 12 October 2015].

[31] T. L. Fagerson, "The Hip Handbook," Butterworth-Heinemann,London, 1998.

[32] J. Katz, E. Losina, J. Barrett, C. Phillips, N. Mahomed, R. Lew, E. Guadagnoli, W. Harris, R. Poss and J. Baron, "Association between hospital and surgeon procedure volume and outcomes of total hip replacement in the United States medicare population," Bone Joint Surg Am, Vols. 83-A, pp. 1622-1629, 2001.

[33] D. Felson and Y. Zhang, "An update on the epidemiology of knee and hip osteoarthritis with a view to prevention," Arthritis Rheum, vol. 41, pp. 13431355., 1998.

[34] J. Jordan, G. Linder, J. Renner and J. Fryer, "The impact of arthritis in rural populations," Arthritis Care Res , vol. 8, pp. 242-250, 1995.

[35] M. Nevitt, L. Xu, Y. Zhang, L. Lui, W. Yu, N. Lane, M. Qin, M. Hochberg, S. Cummings and D. Felson, "Very low prevalence of hip osteoarthritis among Chinese elderly in Beijing, China, compared with whites in the United States: the Beijing osteoarthritis study," Arthritis Rheum 2002, vol. 46, pp. 17731779, 2002.

[36] A. Maetzel, M. Makela, G. Hawker and C. Bombardier, "Osteoarthritis of the hip and knee and mechanical occupational exposure--a systematic overview of the evidence," J Rheumatolgy, vol. 24, pp. 1599-1607, 1997.

[37] C. Cooper, H. Inskip, P. Croft, L. Campbell, G. Smith, M. McLaren and D. Coggon, "Individual risk factors for hip osteoarthritis: obesity, hip injury, and physical activity," Am J Epidemiol , vol. 147, pp. 516-522, 1998.

[38] P. Croft, D. Coggon, M. Cruddas and C. Cooper, "Osteoarthritis of the hip: an occupational disease in farmers," BMJ , vol. 304, pp. 1269-1272, 1992.

[39] P. Croft, C. Cooper, C. Wickham and D. Coggon, "Osteoarthritis of the hip and occupational activity," Scand J Work Environ Health, vol. 18, pp. 59-63, 1992. 
[40] D. J. Hunter, D. \&. Schofield and E. Callander, "The individual and socioeconomic impact of osteoarthritis," Nature Reviews Rheumatology, p. 437-441, 2014.

[41] R. Lawrence, D. Felson, C. Helmick, L. Arnold, H. Choi, R. Deyo, S. Gabriel, R. Hirsch, M. Hochberg, G. Hunder, J. Jordan, J. Katz, H. Kremers and F. Wolfe, "Estimates of the prevalence of arthritis and other rheumatic conditions in the United States. Part II," Arthritis Rheum, pp. 26-35, 2008.

[42] Y. Zhang, J. Niu, M. Kelly-Hayes, C. Chaisson, P. Aliabadi and D. Felson, "Prevalence of symptomatic hand osteoarthritis and its impact on functional status among the elderly," The Framingham Study. Am. J. Epidemiol, p. 10211027, 2002.

[43] C. Vinciguerra, A. Gueguen, M. Revel, J. Heuleu, B. Amor and M. Dougados, "Predictors of the need for total hip replacement in patients with osteoarthritis of the hip," Rev Rhum Engl Ed , vol. 62, pp. 563-570, 1995.

[44] "Dissemination of IT for the promotion of Materials Science and University of Cambridge," [Online]. Available: http://www.doitpoms.ac.uk/tlplib/bones/hip.php. [Accessed 4 March 2015].

[45] "Dissemination of IT for the promotion of Materials Science and University of Cambridge," [Online]. Available: http://www.doitpoms.ac.uk/tlplib/bones/stem.php. [Accessed 20 August 2015].

[46] P. S. \&. Walker and B. L. Gold, "The tribology (friction, lubrication and wear) of all-metal artificial hip joints," Wear, vol. 17, pp. 285-299., 1971.

[47] J. Charnley, "Long-term results of low-friction arthroplasty," Hip, p. 429, 1982.

[48] P. Boutin, P. Christel, J. M. Dorlot, A. Meunier, A. Deroquancourt, D. Blanquaert, S. Herman, L. \&. Sedel and J. Witvoet, "The Use of Dense Alumina Alumina Ceramic Combination in Total Hip-Replacement," Journal of Biomedical Materials Research, vol. 22, pp. 1203-1232, 1988.

[49] P. Bizot, R. Nizard, S. Lerouge, F. \&. Prudhommeaux and L. Sedel, "Ceramic/ceramic total hip arthroplasty," Journal of Orthopaedic Science: Official Journal of the Japanese Orthopaedic Association, vol. 5, pp. 622-627, 2000.

[50] B. Cales, "Zirconia as a sliding material - Histologic, laboratory, and clinical data," Clinical Orthopaedics and Related Research, pp. 94-112, 2000.

[51] P. J. Firkins, J. L. Tipper, E. Ingham, M. H. Stone, R. \&. Farrar and J. Fisher, "A novel low wearing differential hardness, ceramic-on-metal hip joint prosthesis," J Biomech, vol. 34, pp. 1291-1298, 2001b. 
[52] S. Williams, A. Schepers, G. Isaac, C. Hardaker, E. Ingham, D. V. Jagt, A. \&. Breckon and J. Fisher, "THE 2007 OTTO AUFRANC AWARD: Ceramic-onMetal Hip Arthroplasties: A Comparative In Vitro and In Vivo Study," Clin Orthop Relat Res, vol. 465, pp. 23-32, 2007a.

[53] M.-S. Scholz, J. Blanchfield, L. Bloom, B. Coburn, M. Elkington, J. Fuller, M. Gilbert, S. Muflahi, M. Pernice, S. Rae, J. Trevarthen, S. White, P. Weaver and I. Bond, "The use of composite materials in modern orthopaedic medicine and prosthetic devices: A review," Composites Science and Technology 71, pp. 1791-1803, 2011.

[54] H. .. Zhang, "The Influence of Stem Design and Fixation Methods on the Lifetime of Total Hip Replacement," Doctoral thesis, University of Huddersfield, 2009.

[55] E. J. Barbero, Introduction to Composite materials Design, Boca Raton, FL: CRC press, 2011.

[56] P. Tan, L. Tong and G. P. Steven, "Micro mechanics models for mechanical and thermo mechanical properties of 3D through-the-thickness angle interlock woven composites," Composites: Part A 30, p. 637-648, 1999.

[57] Y. Wang and Y. Xia, "Experimental and theoretical study on the strain rate and temperature dependence of mechanical behaviour of Kevlar fibre," Composites: Part A 30, p. 1251-1257, 1999.

[58] A. Aboshio, S. Green and J. Ye, "Experimental investigation of the mechanical properties of neoprene coated nylon woven reinforced composites," Composite Structures 120, p. 386-393, 2015.

[59] D.-s. Li, C.-q. .. Zhao, T.-q. Ge, L. Jiang, C.-j. Huang and N. Jiang, "Experimental investigation on the compression properties and failure mechanism of 3D braided composites at room and liquid nitrogen temperature," Composites: Part B 56 , p. 647-659, 2014.

[60] M. Pankow, A. Salvi, A. Waas, C. Yen and S. Ghiorse, "Split Hopkinson pressure bar testing of 3D woven composites," Composites Science and Technology, vol. 71, p. 1196-1208, 2011.

[61] J. LeBlanc, A. Shukla, C. .. Rousseau and A. Bogdanovich, "Shock loading of three-dimensional woven composite materials," Composite Structures 79, p. 344-355, 2007.

[62] T. Walter, G. Subhash, B. Sankar and C. Yen, "Monotonic and cyclic short beam shear

response of 3D woven composites," Composites Science and Technology 70, p. 2190-2197, 2010. 
[63] Y. Mahadik and S. Hallett, "Finite element modelling of tow geometry in 3D woven fabrics," Elsevier Composites Part A 41, p. 1192-1200, 2010.

[64] Y. Wang and X. Sun, "Digital-element simulation of textile processes," Composites Science and Technology, p. 311-319, 2001.

[65] Y. Wang, Y. Miao and B. Cheeseman, "Digital element method for simulating impact and penetration of textiles," Annual Conference of Fiber Society, 2006.

[66] E. V. Iarve, "Spline variational three dimensional stress analysis of laminated composite plates with open holes," Int. J. Solids Structures Vol. 33. No. 14, pp. 2095 - 2118, 1996.

[67] R. K. Burla, "Finite Element Analysis using uniform b-spline approximation and implicit boundary method," Ph D Thesis, 2008.

[68] G. Farin, Curves and Surfaces for CAGD: A Practical Guide, San Francisco: Morgan Kaufmann Publishers, 2002.

[69] T. Hughes, J. Cottrell and Y. Bazilevs, "Isogeometric analysis: CAD, finite elements,NURBS, exact geometry and mesh refinement.," Computer Methods in Applied Mechanics and Engineering, vol. 194, pp. 4135-4195, 2005.

[70] E. V. Iarve, D. H. Mollenhauer, E. G. Zhou, T. Breitzman and T. J. Whitney, "Independent mesh method-based prediction of local and volume average in textile composites," Elsevier - Composites:Part A 40, pp. 1880-1890, 2009.

[71] G. Zhou, X. Sun and Y. Wang, "Multi-chain digital element analysis in textile mechanics,," Elsevier - Composites Science and Technology 64, pp. 239-244, 2004.

[72] G. Zhou, X. Sun and Y. Wang, "Multi-chain digital element analysis in textile mechanics," Elsevier - Composites Science and Technology 64, pp. 239-244, 2003.

[73] A. Mouritz, M. Bannister, P. Falzon and K. Leong, "Review of applications for advanced three-dimensional fibre textile composite," Composites: Part A 30, April, p. 1445 - 1461, 1999.

[74] E. Zhou, D. Mollenhauer and L. Endel, "Image Reconstruction Based Modeling of 3D Textile Composite (Postprint)," Dayton University, OH Research Institute, Ft. Belvoir Defense Technical Information Center,, pp. AFRL-ML-WP-TP-2007-442., 2007.

[75] A. Oshkour, N. A. Osman, M. Bayat, R. Afshar and F. Berto, "Threedimensional finite element analyses of functionally graded femoral prostheses with different geometrical configurations," Materials and Design 56 , p. 9981008, 2014. 
[76] E. J. Barbero, Finite Element Analysis of Composite Materials using Abaqus, Boca Raton: CRC Press, 2013.

[77] C. J. Lavernia, D. A. Iacobelli, J. M. Villa, J. Kinzy, J. L. Gonzalez and W. J. Kinzy, "Trunnion-Head Stresses in THA: Are Big Heads Trouble?," The Journal of Arthroplasty, vol. 30, no. 6, p. 1085-1088, 2015.

[78] W. Hodge, R. Fijan, K. Carlson, R. Burgess, W. Harris and R. Mann, "Contact pressures in the human hip joint measured in vivo," Proc Natl Acad Sci U S A., pp. 2879-2883, 1986.

[79] B. Pal, P. Puthumanapully and A. Amis, "Biomechanics of implant fixation," Orthopaedics and Trauma, vol. 27, pp. 76-84, 2013.

[80] R. Ruben, J. Folgado and P. Fernandes, "Three-dimensional shape optimization of hip prosthesesusing a multicriteria formulation," Structural and Multidisciplinary Optimization, vol. 34, pp. 261-275, 2007.

[81] R. Karkkainen and T. Jerome, "Micromechanical Strength Modeling and Investigation of Stitch Density Effects on 3D-Orthogonal Composites," Journal of Composite Materials, vol. 43, no. 25, pp. 3125-3142, 2009.

[82] R. Karkkainen and C.-F. Yen, "Dynamic Modeling for Rate Dependent and Mode Dependent Cohesive Interface Failure Analysis," Journal of Composite Materials, vol. 46, no. 18, pp. 2193-2202, 2012. 


\section{Appendix}

\section{VTMS $\backslash B S A M$ Code:}

Sample Input file generated from VTMS to perform analysis in BSAM(shown only partial code... deleting nodes, elements etc)

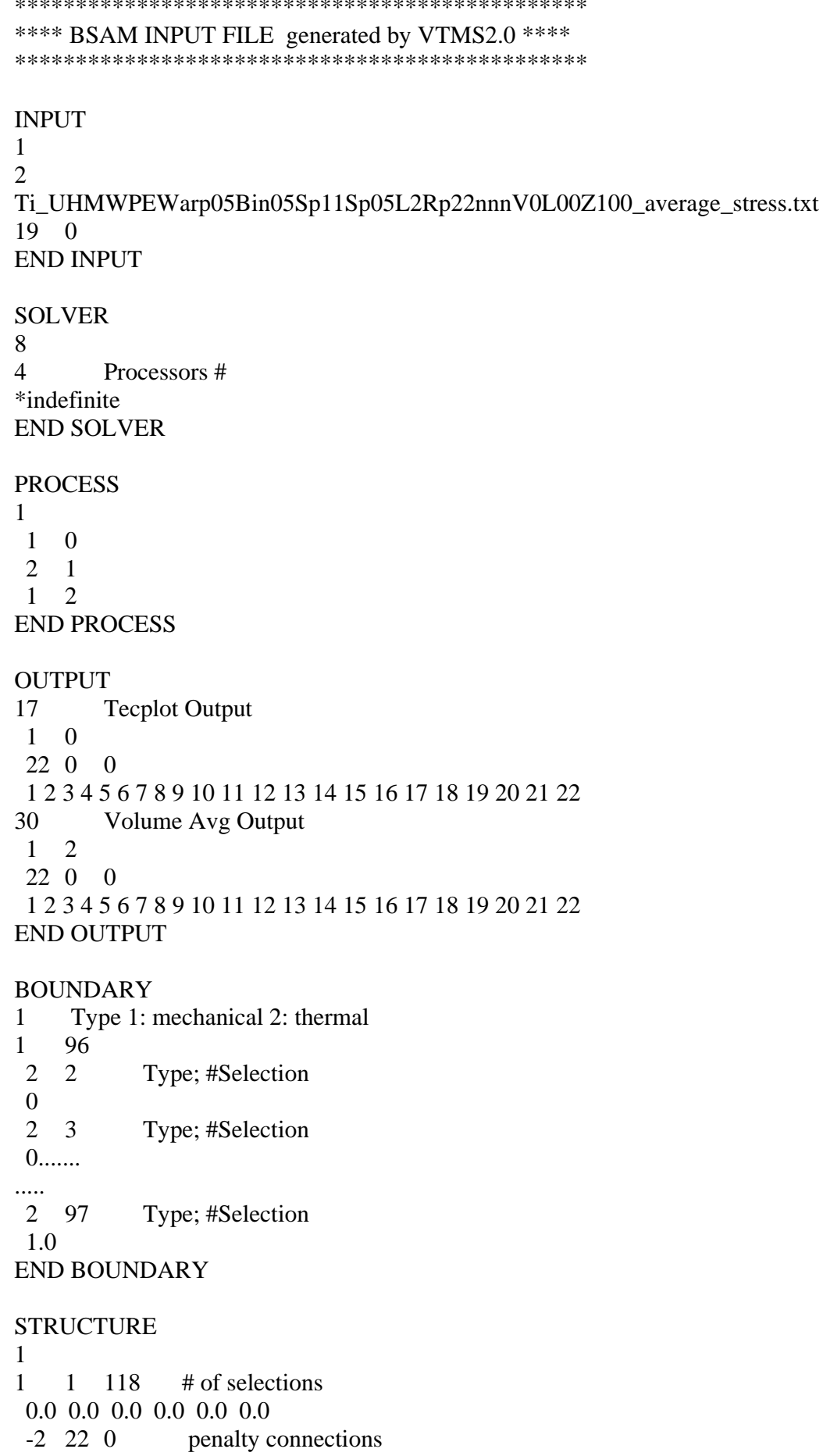




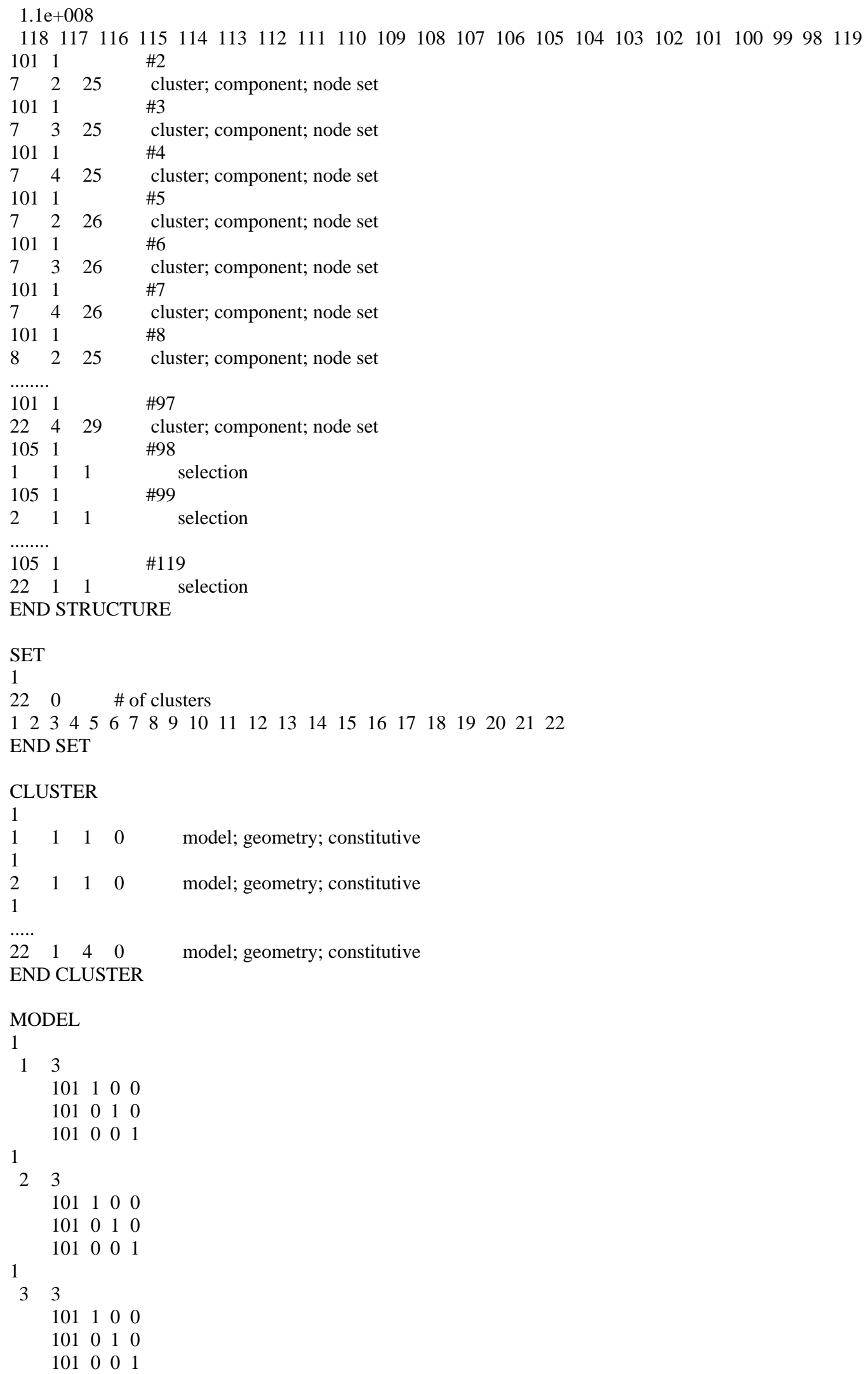




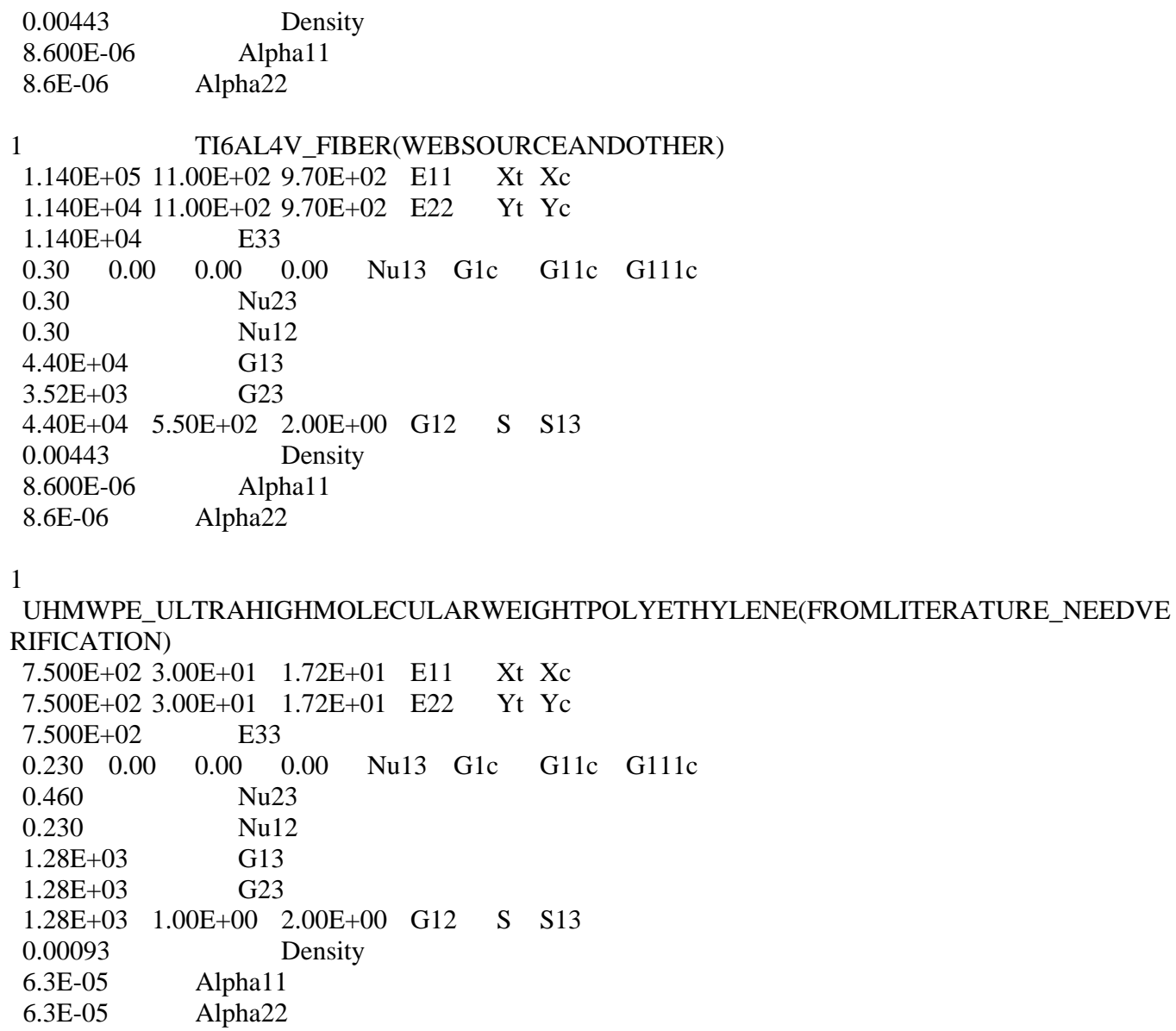

END MATERIAL

APPROXIMATION

100

**** No.1 Entity*****

$* * * *$ File name $=$ 1BIN1WARP05BIN05SP11SP05L2RP22NNN.ELE ******

*DIMENSION

$4275, \quad 3584, \quad 26,0$

*NODE

$1, \quad 0.200000002, \quad 2.08026857, \quad 0.996597201$

$2, \quad 0.200000001, \quad 2.01784791, \quad 0.91951247$

$3, \quad 0.2, \quad 1.95780421, \quad 0.845595361$

$\cdots \cdot$

….

3305, $\quad 5.75, \quad 2.38026697, \quad 1.28776116$

3306, $\quad 5.75, \quad 2.4392938, \quad 1.38263124$

*ELEMENT, TYPE=X3D8

$1, \quad 1,2,7,6,58,59,64,63$

$2735, \quad 3245, \quad 3246, \quad 3249, \quad 3248, \quad 3302, \quad 3303, \quad 3306, \quad 3305$

2736, 3246, 3242, 3243, 3249, 3303, 3299, 3300, 3306 
*Nset, nset $=$ PSET-0

$1, \quad 2,3,4,5,6,7,8,9,10,11,12,13,14,15,16$,

….

3289, 3290, 3291, 3292, 3293, 3294, 3295, 3296, 3297, 3298, 3299, 3300, 3301,

3302, 3303, 3304,

$3305, \quad 3306$

*STOP

100

**** No.3 Entity*****

$* * * *$ File name $=3$ BIN3WARP05BIN05SP11SP05L2RP22NNN.ELE $* * * * * *$

$\cdots \cdots$

100

**** No.4 Entity*****

$* * * *$ File name $=$ 4BIN4WARP05BIN05SP11SP05L2RP22NNN.ELE $* * * * * *$

**** No.5 Entity*****

$* * * *$ File name $=5$ BIN5WARP05BIN05SP11SP05L2RP22NNN.ELE $* * * * * *$

**** No.6 Entity*****

$* * * *$ File name $=6$ BIN6WARP05BIN05SP11SP05L2RP22NNN.ELE $* * * * * *$

....

**** No.7 Entity*****

$* * * *$ File name $=$ 7WEFT1WARP05BIN05SP11SP05L2RP22NNN.ELE $* * * * * *$

....

**** No.8 Entity*****

$* * * *$ File name $=8$ WEFT2WARP05BIN05SP11SP05L2RP22NNN.ELE $* * * * * *$

**** No.22 Entity*****

$* * * *$ File name $=$ MAT1.ELE $* * * * * *$

*DIMENSION

137600, 109783, 29, 0

*NODE

$\begin{array}{llll}1, & 0.200000003, & 0.800000012, & 0.200000003\end{array}$

2, $\quad 0.292500019, \quad 0.800000012, \quad 0.200000003$

3, $\quad 0.38500002, \quad 0.800000012, \quad 0.200000003$

137599, 5.65750027, $\quad 3.20000005, \quad 2.4000001$

$137600, \quad 5.75, \quad 3.20000005, \quad 2.4000001$

*ELEMENT, TYPE=X3D8

$1, \quad 1,2,63,62,2376,2377,2438,2437$

$\ldots$

39705, 48412, 48413, 48471, 48470, 50706, 50707, 50768, 50767

109581, 109582, 109583, 109584, 109585, 109586, 109587, 109643, 109644, 109645, 109646

*Elset, elset=Conn-11

1671, 1672, 1673, 1674, 1714, 1715, 1716, 1717, 1753, 1754, 1755, 1794, 1795, 1839, 1840, 1890,

...

107858, 107859, 107860, 107912, 107913, 107914, 107915, 107916, 107917, 107918, 107919, 107920, 107973, 107974, 107975, 107976,

107977, 107978, 107979, 108034, 108035, 108036, 108037, 108038, 108096 
*Elset, elset=Conn-22

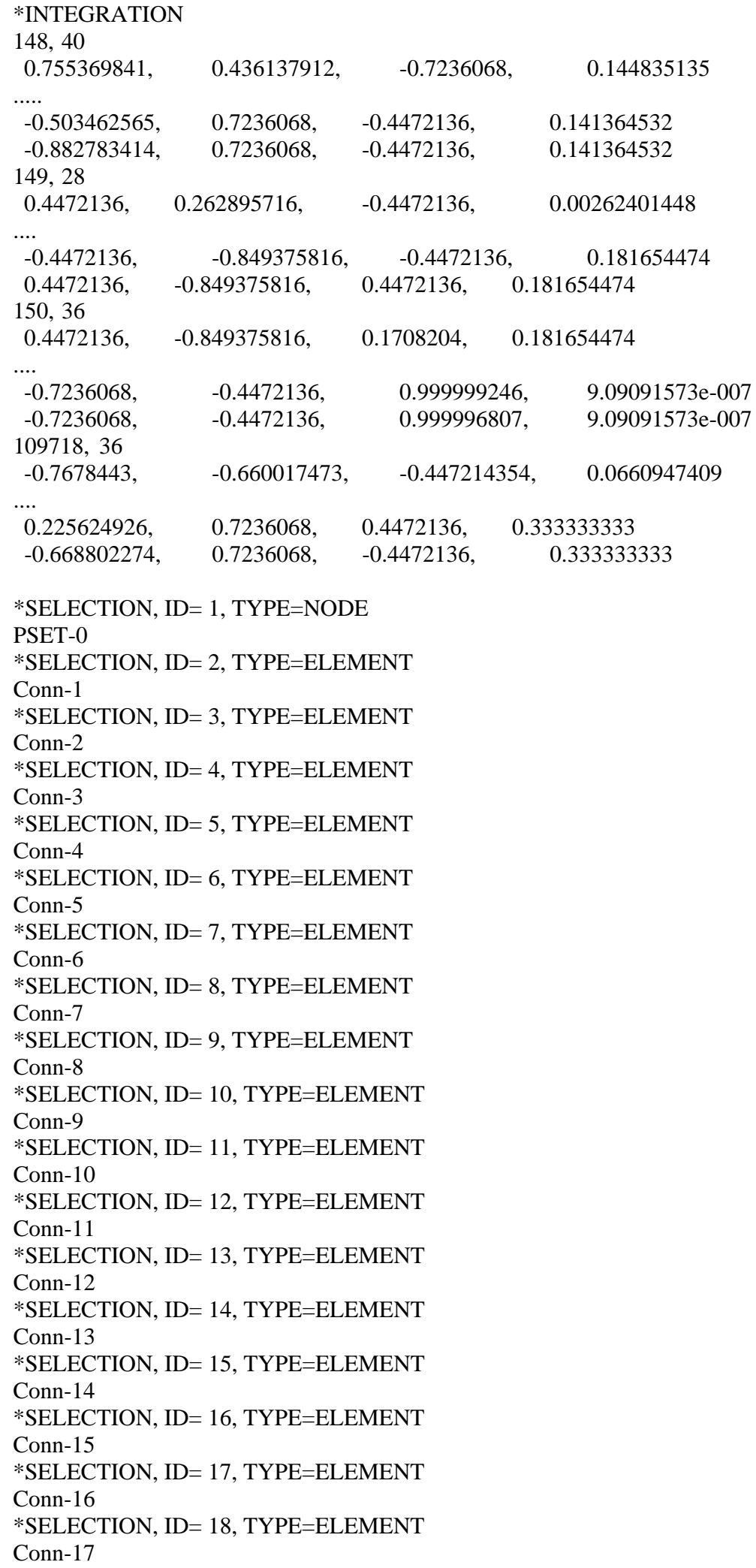


*SELECTION, ID= 19, TYPE=ELEMENT

Conn-18

*SELECTION, ID= 20, TYPE=ELEMENT

Conn-19

*SELECTION, ID= 21, TYPE=ELEMENT

Conn-20

*SELECTION, ID= 22, TYPE=ELEMENT

Conn-21

*SELECTION, ID= 23, TYPE=ELEMENT

Conn-22

*SELECTION, ID= 24, TYPE=NODE

PSET-1

*SELECTION, ID= 25, TYPE=NODE

PSET-2

*SELECTION, ID= 26, TYPE=NODE

PSET-3

*SELECTION, ID= 27, TYPE=NODE

PSET-4

*SELECTION, ID= 28, TYPE=NODE

PSET-5

*SELECTION, ID= 29, TYPE=NODE

PSET-6

*STOP

END APPROXIMATION

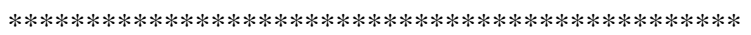

**** END BSAM INPUT FILE $* * * * * * * * * * * * * * * * * * * * *$

$* * * * * * * * * * * * * * * * * * * * * * * * * * * * * * * * * * * * * * * * * * * * * * *$ 


\section{Engineering Drawing of Hip Implants}

1. Stem using Profile1

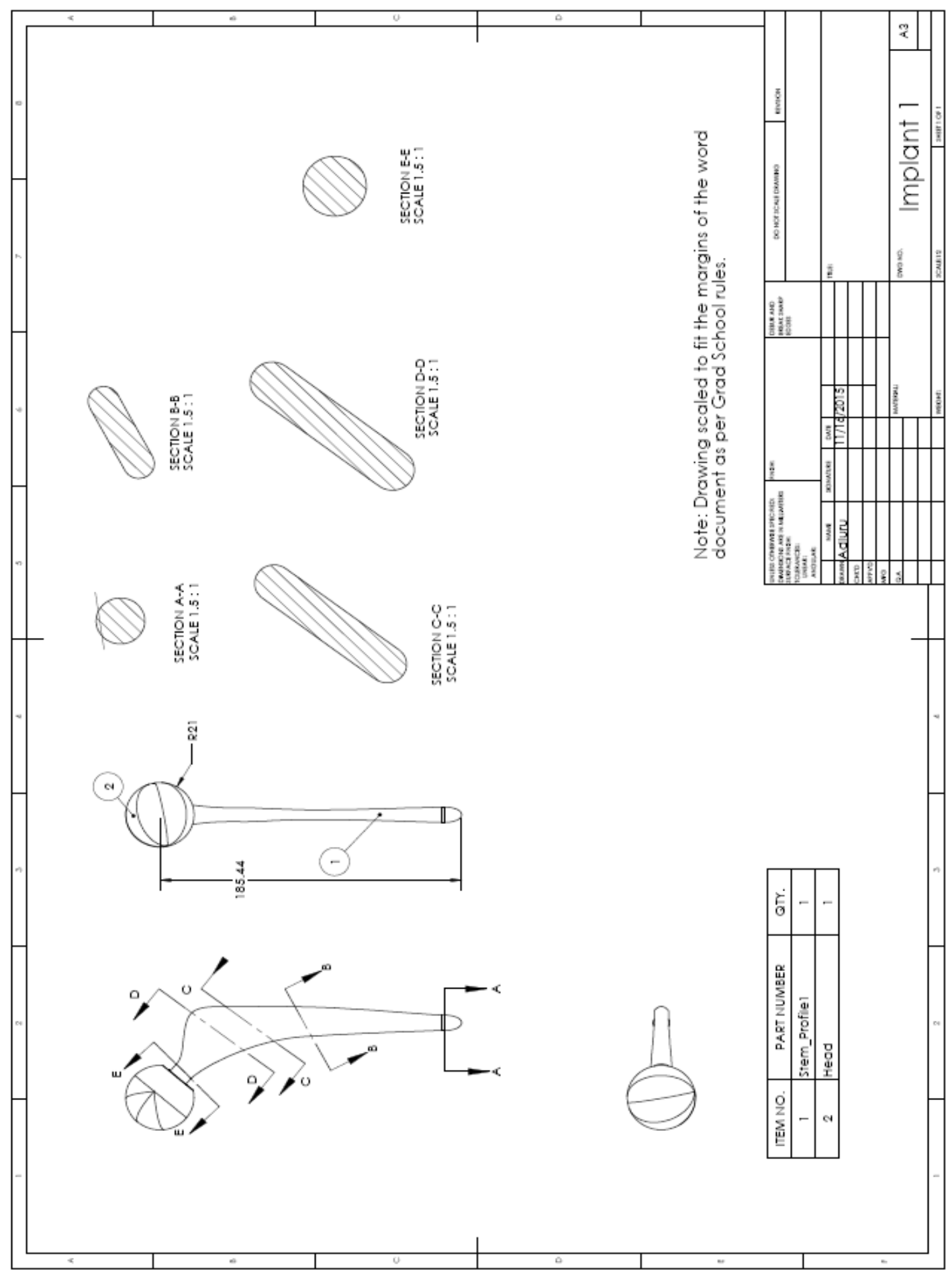


2. Stem using Profile2

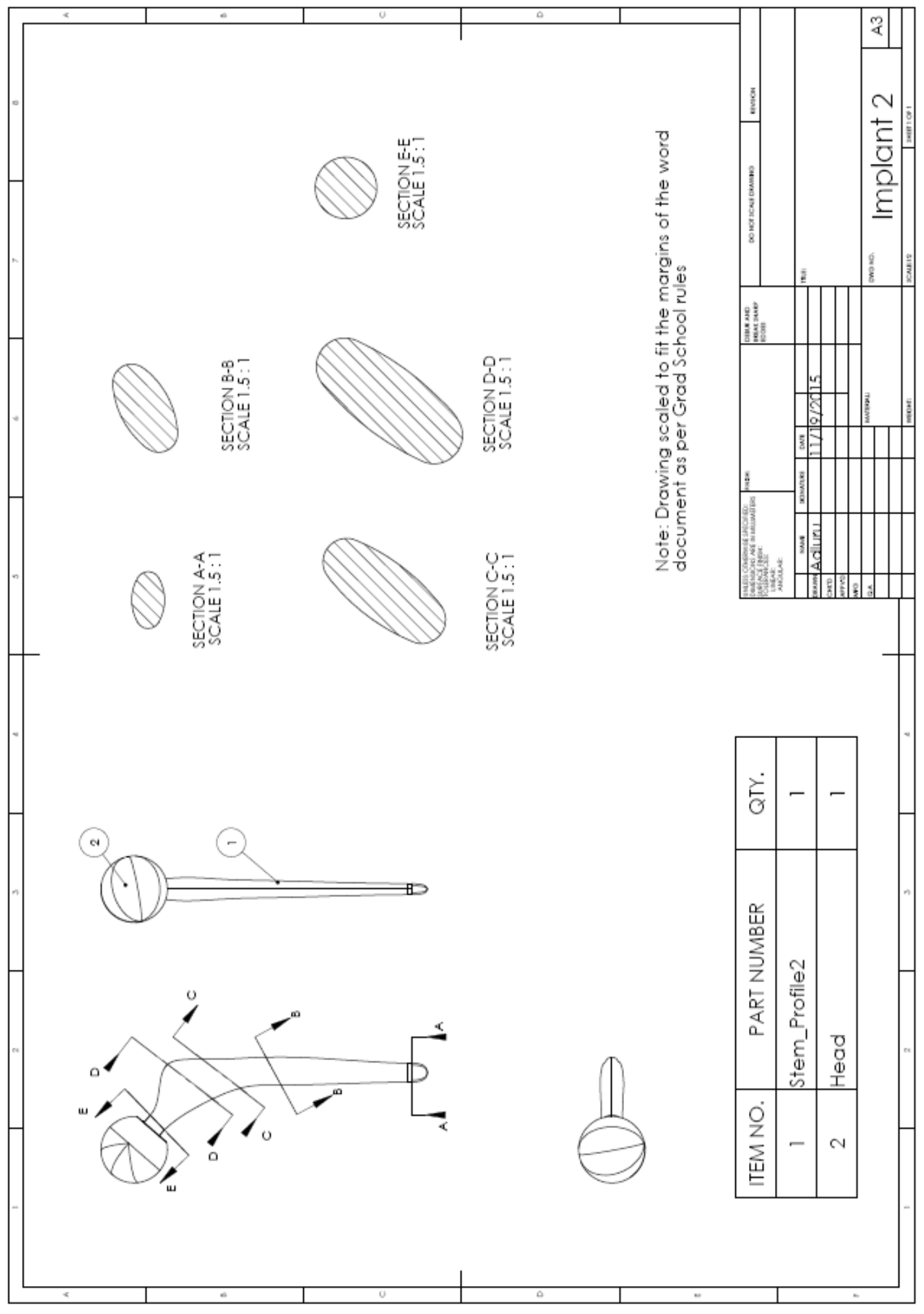




\section{Hypermesh $\backslash$ Abaqus Code:}

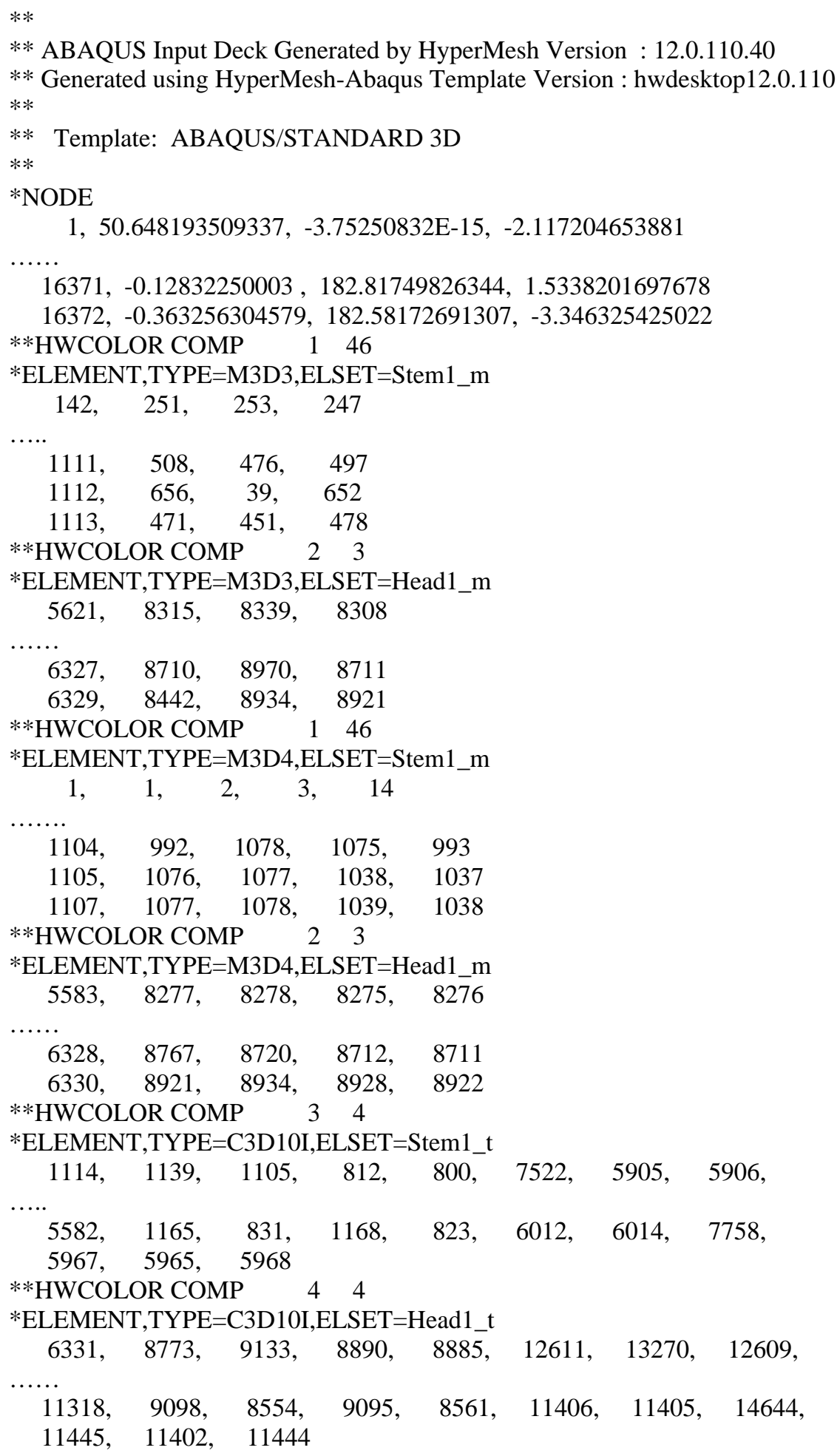




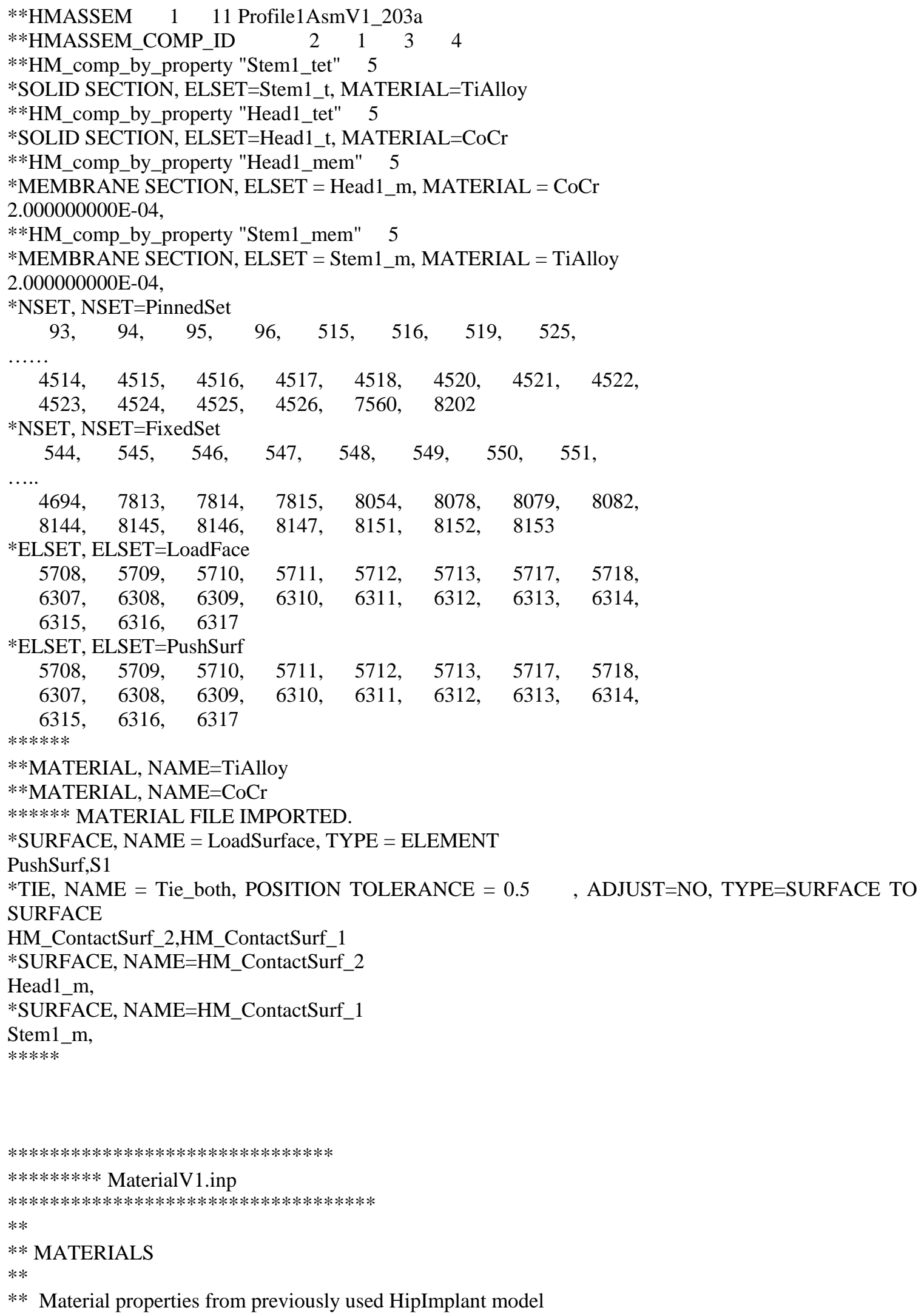




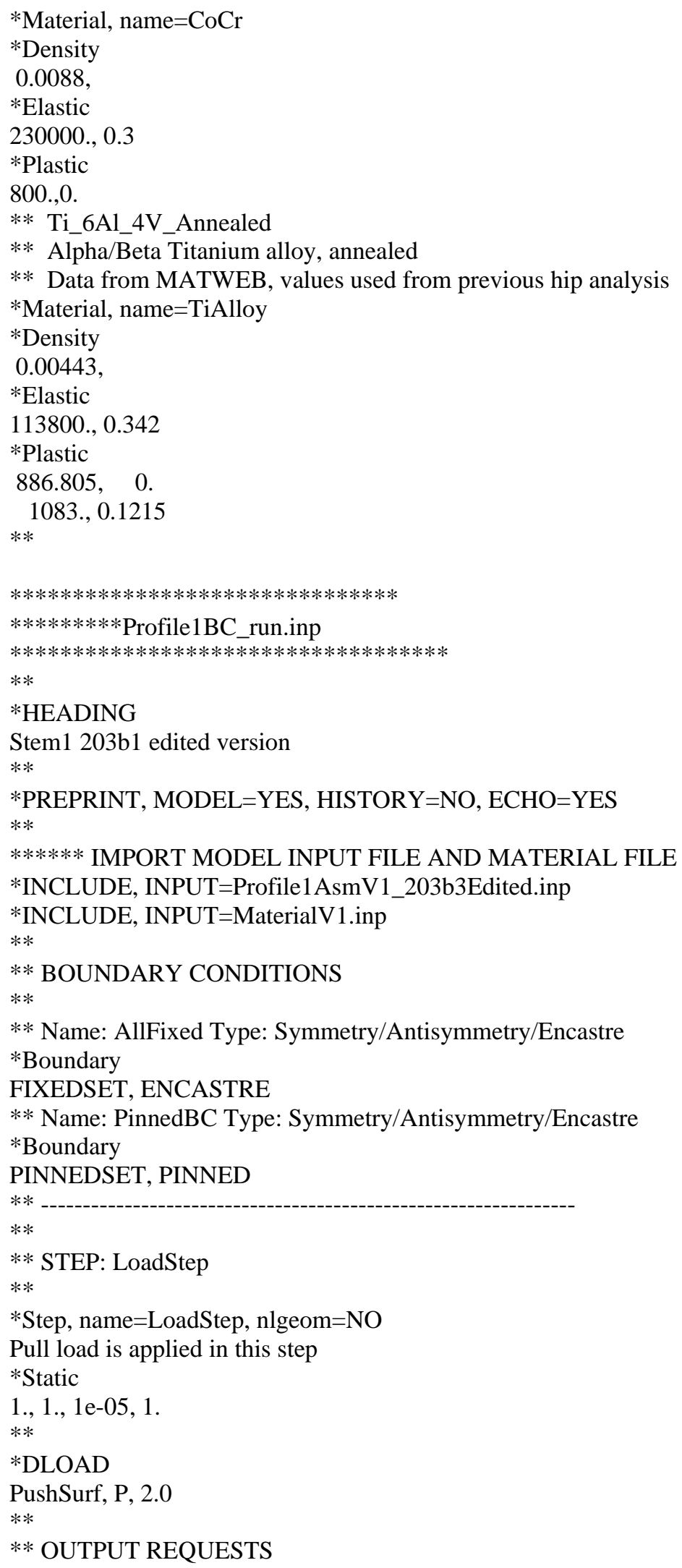


$* *$

$*$ Restart, write, frequency $=0$

**

** FIELD OUTPUT: F-Output-1

**

*Output, field, variable=PRESELECT

**

** HISTORY OUTPUT: H-Output-1

$* *$

*Output, history, variable=PRESELECT

*End Step 


\section{Table A1 Master Table with all the architectures used for analysis}

\begin{tabular}{|c|c|c|c|c|}
\hline Architecture & Orientation & $\begin{array}{l}\text { Fiber } \\
\text { Material }\end{array}$ & $\begin{array}{l}\text { Matrix } \\
\text { Material }\end{array}$ & $\begin{array}{l}\text { Young's } \\
\text { Modulus(GPa) }\end{array}$ \\
\hline 3DOW & $0^{\circ}$ & $\mathrm{CF}$ & UHMWPE & 23.02 \\
\hline 3DOW & $0^{\circ}$ & $\mathrm{CF}$ & UHMWPE & 53.82 \\
\hline 3DOW & $0^{\circ}$ & $\mathrm{CoCr}$ & UHMWPE & 22.04 \\
\hline 3DOW & $0^{\circ}$ & $\mathrm{CF}$ & PEEK & 26.82 \\
\hline 3DOW & $0^{\circ}$ & $\mathrm{CoCr}$ & PEEK & 25.78 \\
\hline 3DOW & $0^{\circ}$ & Ti6Al4V & PEEK & 15.389 \\
\hline P2P ILw/o W & $0^{\circ}$ & $\mathrm{CF}$ & UHMWPE & 81.42 \\
\hline P2P ILw/o W & $90^{\circ}$ & CF & UHMWPE & 18.34 \\
\hline P2P ILw/o W & $0^{\circ}$ & $\mathrm{CoCr}$ & UHMWPE & 35.11 \\
\hline P2P ILw/o W & $90^{\circ}$ & $\mathrm{CoCr}$ & UHMWPE & 16.11 \\
\hline P2P ILw/o W & $90^{\circ}$ & Ti6Al4V & UHMWPE & 4.25 \\
\hline P2P ILw/o W & $0^{\circ}$ & $\mathrm{CF}$ & PEEK & 60.931 \\
\hline P2P ILw/o W & $90^{\circ}$ & $\mathrm{CF}$ & PEEK & 11.562 \\
\hline P2P ILw/o W & $0^{\circ}$ & $\mathrm{CoCr}$ & PEEK & 40.239 \\
\hline P2P ILw/o W & $90^{\circ}$ & $\mathrm{CoCr}$ & PEEK & 23.148 \\
\hline P2P ILw/o W & $0^{\circ}$ & Ti6Al4V & PEEK & 24.021 \\
\hline P2P ILw/o W & $90^{\circ}$ & Ti6Al4V & PEEK & 16.087 \\
\hline P2P Ang IL & $0^{\circ}$ & CF & UHMWPE & 47.82 \\
\hline P2P Ang IL & $90^{\circ}$ & $\mathrm{CF}$ & UHMWPE & 3.17 \\
\hline P2P Ang IL & $0^{\circ}$ & $\mathrm{CoCr}$ & UHMWPE & 58.95 \\
\hline P2P Ang IL & $90^{\circ}$ & $\mathrm{CoCr}$ & UHMWPE & 5.88 \\
\hline P2P Ang IL & $0^{\circ}$ & Ti6Al4V & UHMWPE & 31.85 \\
\hline P2P Ang IL & $90^{\circ}$ & Ti6Al4V & UHMWPE & 4.25 \\
\hline P2P Ang IL & $0^{\circ}$ & $\mathrm{CF}$ & PEEK & 60.931 \\
\hline P2P Ang IL & $90^{\circ}$ & $\mathrm{CF}$ & PEEK & 11.562 \\
\hline P2P Ang IL & $0^{\circ}$ & $\mathrm{CoCr}$ & PEEK & 62.943 \\
\hline P2P Ang IL & $90^{\circ}$ & $\mathrm{CoCr}$ & PEEK & 11.379 \\
\hline
\end{tabular}




\begin{tabular}{rcccc}
\hline Architecture & Orientation & $\begin{array}{l}\text { Fiber } \\
\text { Material }\end{array}$ & $\begin{array}{l}\text { Matrix } \\
\text { Material }\end{array}$ & $\begin{array}{c}\text { Young's } \\
\text { Modulus(GPa) }\end{array}$ \\
\hline P2P Ang IL & $0^{\circ}$ & Ti6Al4V & PEEK & 35.104 \\
P2P Ang IL & $90^{\circ}$ & Ti6Al4V & PEEK & 8.609 \\
TriAxial & $0^{\circ}$ & CF & UHMWPE & 33.12 \\
TriAxial & $90^{\circ}$ & CF & UHMWPE & 7.79 \\
TriAxial & $0^{\circ}$ & CoCr & UHMWPE & 42.65 \\
TriAxial & $90^{\circ}$ & CoCr & UHMWPE & 11.32 \\
TriAxial & $0^{\circ}$ & Ti6Al4V & UHMWPE & 25.06 \\
TriAxial & $90^{\circ}$ & Ti6Al4V & UHMWPE & 8.19 \\
TriAxial & $0^{\circ}$ & CF & PEEK & 39.622 \\
TriAxial & $90^{\circ}$ & CF & PEEK & 12.926 \\
TriAxial & $0^{\circ}$ & CoCr & PEEK & 47.969 \\
TriAxial & $90^{\circ}$ & CoCr & PEEK & 17.887 \\
TriAxial & $0^{\circ}$ & Ti6Al4V & PEEK & 29.561 \\
TriAxial & $90^{\circ}$ & Ti6Al4V & PEEK & 13.627 \\
\hline
\end{tabular}


VITA

\section{HARI KISHORE ADLURU}

Born, Visakhapatnam, Andhra Pradesh, INDIA

1996-2000 B.E., Mechanical Engineering

A. U. College of Engineering, Andhra University

Visakhapatnam, Andhra Pradesh

2001-2004 M.S., Mechanical Engineering

Florida International University

Miami, Florida

2001-2004 Teaching and Research Assistant

Florida International University

Miami, Florida

2003-2008 Mechanical Intern, Mechanical Engineer, Sr. Mechanical Engineer, Project Management Officer Applica Consumer Products Inc, Miramar, Florida

2008 Six sigma green belt

Microsoft certified project professional

2009 Lecturer, Manipal Institute of Technology

Manipal University

Udupi, Karnataka, India

2010-2015 Teaching, Graduate and Research Assistant, Instructor Florida International University

Miami, Florida

2011 \& $2013 \quad$ Optimization Design Engineer

Entic USA LLC

Pembroke Pines, Florida

2014 -2015 Doctoral Candidate

Florida International University

Miami, Florida

PATENTS, PUBLICATIONS AND PRESENTATIONS 
“In-situ Optimization of Chilled Water Plants” USPTO\# 20140229146, Filed: Feb 2013, Issued Aug 2014 Inventors: Igor F. Gonzalez, Hari Kishore Adluru, Aparna Aravelli

H. Adluru; Kinzy W. Jones; A. Aravelli; Singiresu S. Rao "Design Optimization and Analysis of Micro-channel Heat Exchanger embedded in LTCC”, IMAPS 2013 Southeastern Microelectornic Packaging Conference - Feb 2013 Orlando, FL

Aravelli, A.; Rao, S. S.; Adluru, H.K. "Multiobjective Design Optimization of Microchannel Cooling System using High Performance Thermal vias in LTCC substrates”, Journal of Microelectronics and Electronic Packaging 2013 vol 10, pp: 40-47

Aravelli, A.; Rao, S. S.; Adluru, H.K. "Design Optimization of Micro-channel Heat Exchanger embedded in LTCC”, Proceedings of 2012 of the 45th International Symposium on Microelectronics, IMAPS, San Diego, USA, pp: 857-865 (Best Paper in Session)

Marc A. Zampino; Hari Adluru; Yanquing Liu; W. Kinzy Jones; "LTCC substrates with internal cooling channel and Heat Exchangers," Proceedings of 2003 of the 36th International Symposium on Microelectronics, IMAPS, Boston, USA, pp: 505-510.

Hari Adluru; Marc A. Zampino; Yanqing Liu; W. Kinzy Jones; "Embedded Heat Exchanger in LTCC Substrate", Conference: Ceramic Interconnect Technology: The Next Generation, Denver, CO; Proceedings of SPIE-The International Society for Optical Engineering, 2003, v. 5231, p. 205-210 